\title{
Iron-Catalyzed Oxyfunctionalization of Aliphatic Amines at Remote Benzylic C-H Sites
}

\author{
Curren T. Mbofana, Eugene Chong, James Lawniczak and Melanie S. Sanford* \\ Department of Chemistry, University of Michigan, 930 North University Ave, Ann Arbor, \\ Michigan 48109, United States \\ mssanfor@umich.edu
}

$\begin{array}{ll}\text { I. General Procedures } & \text { S2 }\end{array}$

$\begin{array}{lr}\text { II. Preliminary Studies and Optimization } & \text { S3 }\end{array}$

$\begin{array}{ll}\text { III. General Procedure I for Amine Methylation } & \text { S8 }\end{array}$

IV. General Procedure II for $N$-Alkylation of Amines $\quad$ S11

$\begin{array}{ll}\text { V. Procedure for } N \text {-Alkylation of Amines 18, } 19 \text { and } 22 & \text { S14 }\end{array}$

VI. General Procedure III for $N$-Alkylation of Amines $\quad$ S16

VII. General Procedure for Catalytic Amine Oxyfunctionalization $\quad$ S17

$\begin{array}{ll}\text { VIII. References } & \text { S26 }\end{array}$

IX. ${ }^{1} \mathrm{H}$ NMR, ${ }^{13} \mathrm{C}$ NMR and ${ }^{19}$ F NMR Spectra $\quad$ S27 


\section{General Procedures}

Proton NMR spectra were recorded on 400 or 500 Varian MHz spectrometers. Proton chemical shifts $(\delta)$ are reported in parts per million (ppm) downfield of tetramethylsilane, using residual solvent as internal standard $\left(\mathrm{CHCl}_{3}, \delta 7.26 \mathrm{ppm}\right)$. Spectral data are reported as follows: chemical shift (multiplicity [singlet (s), broad singlet (br. s), apparent singlet (app. s), doublet (d), broad doublet (br. d), apparent doublet (app. d), doublet of doublets (dd), doublet of triplets (dt), triplet $(\mathrm{t})$, triplet of doublets (td), pentet (p), heptet (hept), multiplet (m), multiple peaks], coupling constants [Hz], integration). Carbon NMR spectra were recorded on a 400 (100) or 500 (126) MHz Varian spectrometers with complete proton decoupling. Carbon chemical shifts $(\delta)$ were reported in parts per million ( $\mathrm{ppm}$ ) downfield of tetramethylsilane, using residual protonated solvent as an internal standard $\left(\mathrm{CDCl}_{3}, \delta 77.2 \mathrm{ppm}\right)$. Infrared spectroscopy (attenuated total reflectance (ATRIR)) was performed on a Perkin-Elmer Spectrum BX Ft-IR spectrometer. Melting point data (mp) were collected on an OptiMelt Automated Melting Point System. Flash column chromatography was performed using Silica Gel $60 \AA$ (32-63 micron) or on a Biotage Isolera ${ }^{\circledR}$ automated chromatography system using SNAP Ultra normal phase cartridges. CV stands for column volume. Peroxide tests were conducted with Quantofix ${ }^{\circledR}$ Peroxide 100 test strips. High resolution mass spectrometry (HRMS) was performed on a Micromass AutoSpec Ultima Magnetic Sector mass spectrometer. The method of ionization is given in parentheses. Gas chromatography-mass spectrometry (GC-MS) analysis was conducted on a Shimadzu GCMS-QP2010 Plus. All catalytic oxidation reactions were run under ambient conditions. 


\section{Preliminary Studies and Optimization}

\section{IIa. Oxidation of 1 Without Acid}

In a 1 dram glass vial charged with a stir-bar, $N, N$-dimethyl phenylbutylamine (1) (44 mg, $0.25 \mathrm{mmol})$ was dissolved in pyridine/MeCN (1:5) $(0.50 \mathrm{~mL}) . \mathrm{FeCl}_{3} \cdot 6 \mathrm{H}_{2} \mathrm{O}(6.8 \mathrm{mg}, 0.025 \mathrm{mmol})$ and 2-picolinic acid $(7.7 \mathrm{mg}, 0.0625 \mathrm{mmol})$ were added and the mixture was stirred until it was homogenous. ${ }^{t} \mathrm{BuOOH}\left(70 \mathrm{wt} \%\right.$ in $\mathrm{H}_{2} \mathrm{O}, 0.10 \mathrm{~mL}, 0.75 \mathrm{mmol}$ ) was added to the vial via syringe in a single batch. The mixture was stirred at room temperature until all the peroxide was consumed, as determined by peroxide test strips $(7 \mathrm{~h})$. The mixture was concentrated in vacuo and dissolved in $\mathrm{MeCN}$. A portion of solid $\mathrm{Na}_{2} \mathrm{CO}_{3}(\sim 200 \mathrm{mg})$ was added to precipitate the Fe catalyst. The mixture was allowed to stand for about $30 \mathrm{~min}$, and then filtered over Celite. An aliquot of this mixture was analyzed by GC-MS.

\section{IIb. Oxidation of 1 With Acid}

To a 1 dram glass vial charged with a stir-bar and $N, N$-dimethyl phenylbutylamine (1) (44 $\mathrm{mg}, 0.25 \mathrm{mmol}), \mathrm{CF}_{3} \mathrm{CO}_{2} \mathrm{H}(21 \mu \mathrm{L}, 0.275 \mathrm{mmol})$ was added, and the vial was tightly capped and allowed to stir for about $5 \mathrm{~min}$. Pyridine $/ \mathrm{MeCN}(1: 5)(0.50 \mathrm{~mL})$ was added to the vial, followed by $\mathrm{FeCl}_{3} \cdot 6 \mathrm{H}_{2} \mathrm{O}(6.8 \mathrm{mg}, 0.025 \mathrm{mmol})$ and 2-picolinic acid $(7.7 \mathrm{mg}, 0.0625 \mathrm{mmol})$ and the mixture was stirred until it was homogenous. ${ }^{t} \mathrm{BuOOH}\left(70 \mathrm{wt} \%\right.$ in $\mathrm{H}_{2} \mathrm{O}, 0.10 \mathrm{~mL}, 0.75 \mathrm{mmol}$ ) was added to the vial via syringe in a single batch. The mixture was stirred at room temperature until all the peroxide was consumed, as determined by peroxide test strips $(9 \mathrm{~h})$. The mixture was concentrated in vacuo and dissolved in $\mathrm{MeCN}$. A portion of solid $\mathrm{Na}_{2} \mathrm{CO}_{3}(\sim 200 \mathrm{mg})$ was added to deprotonate the amine and precipitate the Fe catalyst. The mixture was allowed to stand for about $30 \mathrm{~min}$, and then filtered over Celite. An aliquot of this mixture was analyzed by GC-MS.

\section{IIc. Reaction Optimization}

Reactions were set-up following the procedure in IIb with the conditions specified in Table S1. Most reactions tested negative for peroxide after the specified amount of time. If the peroxide test was positive, the reaction mixture was quenched with a saturated aqueous solution of $\mathrm{Na}_{2} \mathrm{~S}_{2} \mathrm{O}_{3}$ until the peroxide test was negative. The reaction mixture was concentrated in vacuo, and the solids were redissolved in $\mathrm{CDCl}_{3}(1.0 \mathrm{~mL})$. 1,3,5-Trimethoxybenzene (14 $\left.\mathrm{mg}, 0.083 \mathrm{mmol}\right)$ was added as an NMR internal standard. A portion of solid $\mathrm{Na}_{2} \mathrm{CO}_{3}(\sim 200 \mathrm{mg})$ was added to deprotonate the amine and precipitate the Fe catalyst. The mixture was mixed thoroughly and 
allowed to stand for about $30 \mathrm{~min}$, then filtered over Celite directly into an NMR tube. Yields were determined by ${ }^{1} \mathrm{H}$ NMR spectroscopy as shown in Figure $\mathrm{S} 1$.

In certain cases where too much peroxide was present in the reaction mixture after $48 \mathrm{~h}$, NMR yields were not determined, as the quenching procedure required a considerable amount of $\mathrm{Na}_{2} \mathrm{~S}_{2} \mathrm{O}_{3}$ solution that made it difficult to accurately determine NMR yields.

Table S1: Reaction Optimization Parameters

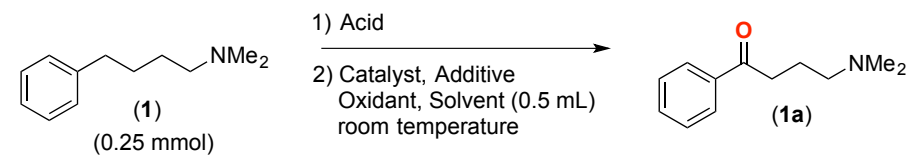

\begin{tabular}{|c|c|c|c|c|c|c|}
\hline Entry & Fe-Catalyst & Picolinic acid & Oxidant & Acid & Solvent & $\begin{array}{l}\text { NMR Yield } \\
{\text { (time) })^{\mathrm{a}}}\end{array}$ \\
\hline 1 & $\mathrm{FeCl}_{3} \cdot 6 \mathrm{H}_{2} \mathrm{O}(6.8 \mathrm{mg}, 10 \mathrm{~mol} \%)$ & $7.7 \mathrm{mg}, 25 \mathrm{~mol} \%$ & $t \mathrm{BuOOH}(0.1 \mathrm{~mL}, 3 \mathrm{eq})$ & $\mathrm{CF}_{3} \mathrm{CO}_{2} \mathrm{H}(21 \mu \mathrm{L}, 1.1 \mathrm{eq})$ & Pyr/MeCN (1:5) & $24 \%(24 h)$ \\
\hline 2 & $\mathrm{FeCl}_{2} \cdot 4 \mathrm{H}_{2} \mathrm{O}(5.0 \mathrm{mg}, 10 \mathrm{~mol} \%)$ & $"$ & $"$ & $"$ & $"$ & $21 \%(24 h)$ \\
\hline 3 & $\mathrm{Fe}(\mathrm{OAc})_{2}(4.3 \mathrm{mg}, 10 \mathrm{~mol} \%)$ & $"$ & $"$ & $"$ & $"$ & $20 \%(24 h)$ \\
\hline 4 & $\mathrm{FeBF}_{4} \cdot 6 \mathrm{H}_{2} \mathrm{O}(8.4 \mathrm{mg}, 10 \mathrm{~mol} \%)$ & $"$ & $"$ & $"$ & $"$ & $23 \%(24 h)$ \\
\hline 5 & $\mathrm{Fe}\left(\mathrm{NO}_{3}\right)_{3} \cdot 9 \mathrm{H}_{2} \mathrm{O}(10 \mathrm{mg}, 10 \mathrm{~mol} \%)$ & $"$ & $"$ & $"$ & $"$ & $18 \%(24 \mathrm{~h})$ \\
\hline 6 & $\mathrm{Fe}(\mathrm{OTf})_{3}(13 \mathrm{mg}, 10 \mathrm{~mol} \%)$ & $"$ & $"$ & $"$ & $"$ & $18 \%(24 \mathrm{~h})$ \\
\hline 7 & $\mathrm{FeCl}_{3} \cdot 6 \mathrm{H}_{2} \mathrm{O}(6.8 \mathrm{mg}, 10 \mathrm{~mol} \%)$ & $"$ & $\mathrm{H}_{2} \mathrm{O}_{2}(77 \mu \mathrm{L}, 3 \mathrm{eq})$ & $"$ & $"$ & $8 \%(24 h)$ \\
\hline 8 & $"$ & $"$ & $t \mathrm{BuOOH}(0.20 \mathrm{~mL}, 6 \mathrm{eq})^{\mathrm{b}}$ & $"$ & $"$ & $38 \%(24 h)$ \\
\hline 9 & $\mathrm{FeCl}_{3} \cdot 6 \mathrm{H}_{2} \mathrm{O}(3.4 \mathrm{mg}, 5 \mathrm{~mol} \%)$ & $3.8 \mathrm{mg}, 12.5 \mathrm{~mol} \%$ & $"$ & $"$ & $"$ & $39 \%(24 \mathrm{~h})$ \\
\hline 10 & $"$ & $"$ & $t \mathrm{BuOOH}(0.41 \mathrm{~mL}, 12 \mathrm{eq})^{\mathrm{c}}$ & $"$ & $"$ & $60 \%(48 h)$ \\
\hline 11 & $\mathrm{FeCl}_{3} \cdot 6 \mathrm{H}_{2} \mathrm{O}(0.7 \mathrm{mg}, 1.0 \mathrm{~mol} \%)$ & $0.8 \mathrm{mg}, 2.5 \mathrm{~mol} \%$ & $"$ & $"$ & $"$ & $63 \%(96 \mathrm{~h})$ \\
\hline 12 & $\mathrm{FeCl}_{3} \cdot 6 \mathrm{H}_{2} \mathrm{O}(3.4 \mathrm{mg}, 5 \mathrm{~mol} \%)$ & $3.8 \mathrm{mg}, 12.5 \mathrm{~mol} \%$ & $"$ & $"$ & $\mathrm{Pyr} / \mathrm{H}_{2} \mathrm{O}(1: 5)$ & $61 \%(72 \mathrm{~h})$ \\
\hline 13 & $"$ & $"$ & $"$ & $"$ & $\mathrm{H}_{2} \mathrm{O}$ & ND \\
\hline 14 & $"$ & $"$ & $"$ & $"$ & $\mathrm{MeCN}$ & ND \\
\hline 15 & $"$ & $"$ & $"$ & $"$ & Pyr & $44 \%(48 \mathrm{~h})$ \\
\hline 16 & $"$ & $"$ & $t \mathrm{BuOOH}(0.20 \mathrm{~mL}, 6 \mathrm{eq})^{\mathrm{b}}$ & $"$ & Pyr/MeCN (1:30) & $40 \%(48 h)^{\star}$ \\
\hline 17 & $"$ & $"$ & tBuOOH $(50 \mu \mathrm{L}, 1.5 \mathrm{eq})$ & $"$ & $\mathrm{MeCN}$ & NR $(24 \mathrm{~h})$ \\
\hline 18 & $"$ & $7.7 \mathrm{mg}, 25 \mathrm{~mol} \%$ & $t \mathrm{BuOOH}(0.41 \mathrm{~mL}, 12 \mathrm{eq})^{\mathrm{c}}$ & $"$ & Pyr/MeCN (1:5) & $61 \%(48 \mathrm{~h})$ \\
\hline 19 & $"$ & $1.9 \mathrm{mg}, 6.25 \mathrm{~mol} \%$ & $"$ & $"$ & $"$ & $64 \%(72 \mathrm{~h})$ \\
\hline 20 & $"$ & None & $"$ & $"$ & $"$ & ND \\
\hline 21 & $"$ & $3.8 \mathrm{mg}, 12.5 \mathrm{~mol} \%$ & $t \mathrm{BuOOH}(0.62 \mathrm{~mL}, 18 \mathrm{eq})^{\mathrm{d}}$ & $"$ & $"$ & $65 \%(48 h)$ \\
\hline 22 & $"$ & $"$ & $"$ & $\mathrm{CF}_{3} \mathrm{CO}_{2} \mathrm{H}(29 \mu \mathrm{L}, 1.5 \mathrm{eq})$ & $"$ & $63 \%(48 \mathrm{~h})$ \\
\hline 23 & $"$ & $"$ & $"$ & $\mathrm{CF}_{3} \mathrm{CO}_{2} \mathrm{H}(38 \mu \mathrm{L}, 2.0 \mathrm{eq})$ & $"$ & $58 \%(48 h)^{*}$ \\
\hline 24 & $"$ & $"$ & $"$ & $\mathrm{CF}_{3} \mathrm{CO}_{2} \mathrm{H}(57 \mu \mathrm{L}, 3.0 \mathrm{eq})$ & $"$ & $51 \%(48 h)^{*}$ \\
\hline 25 & $"$ & $"$ & $"$ & $\mathrm{HBF}_{4}(8 \mathrm{M}, 36 \mu \mathrm{L}, 1.1 \mathrm{eq})$ & $"$ & $61 \%(48 h)^{*}$ \\
\hline 26 & $"$ & $"$ & $"$ & $\mathrm{HCl}(12 \mathrm{M}, 24 \mu \mathrm{L}, 1.1 \mathrm{eq})$ & $"$ & $51 \%(48 \mathrm{~h})$ \\
\hline 27 & $"$ & $"$ & $"$ & $\mathrm{H}_{2} \mathrm{SO}_{4}(18 \mathrm{M}, 15 \mu \mathrm{L} 1.1 \mathrm{eq})$ & $"$ & $40 \%(48 h)^{*}$ \\
\hline 28 & $"$ & $"$ & $"$ & $\mathrm{CH}_{3} \mathrm{CO}_{2} \mathrm{H}(16 \mu \mathrm{L}, 1.1 \mathrm{eq})$ & $"$ & $24 \%(48 h)^{e}$ \\
\hline
\end{tabular}

Unless noted otherwise, reactions were run until they tested negative for peroxide. $\mathrm{Pyr}=$ pyridine. Peroxides: $t \mathrm{BuOOH}\left(70\right.$ wt $\%$ in $\left.\mathrm{H}_{2} \mathrm{O}\right)$ and $\mathrm{H}_{2} \mathrm{O}_{2}(30$ wt \% in $\mathrm{H}_{2} \mathrm{O}$ ) were used in this screen. ${ }^{a} \mathrm{H}-\mathrm{NMR}$ yield determined using trimethoxybenzene as an internal standard. bSlow addition over $5 \mathrm{~h}$. ${ }^{\mathrm{C}} \mathrm{Slow}$ addition over $10 \mathrm{~h}$. dSlow addition over $15 \mathrm{~h}$. *Quenched for peroxide before work-up. ND - yield not determined as too much peroxide was present after $48 \mathrm{~h}$. NR - no reaction e Observed significant decomposition of starting material. 
Figure S1: ${ }^{1}$ H-NMR Spectra of the crude reaction mixture in Table S1, entry 21

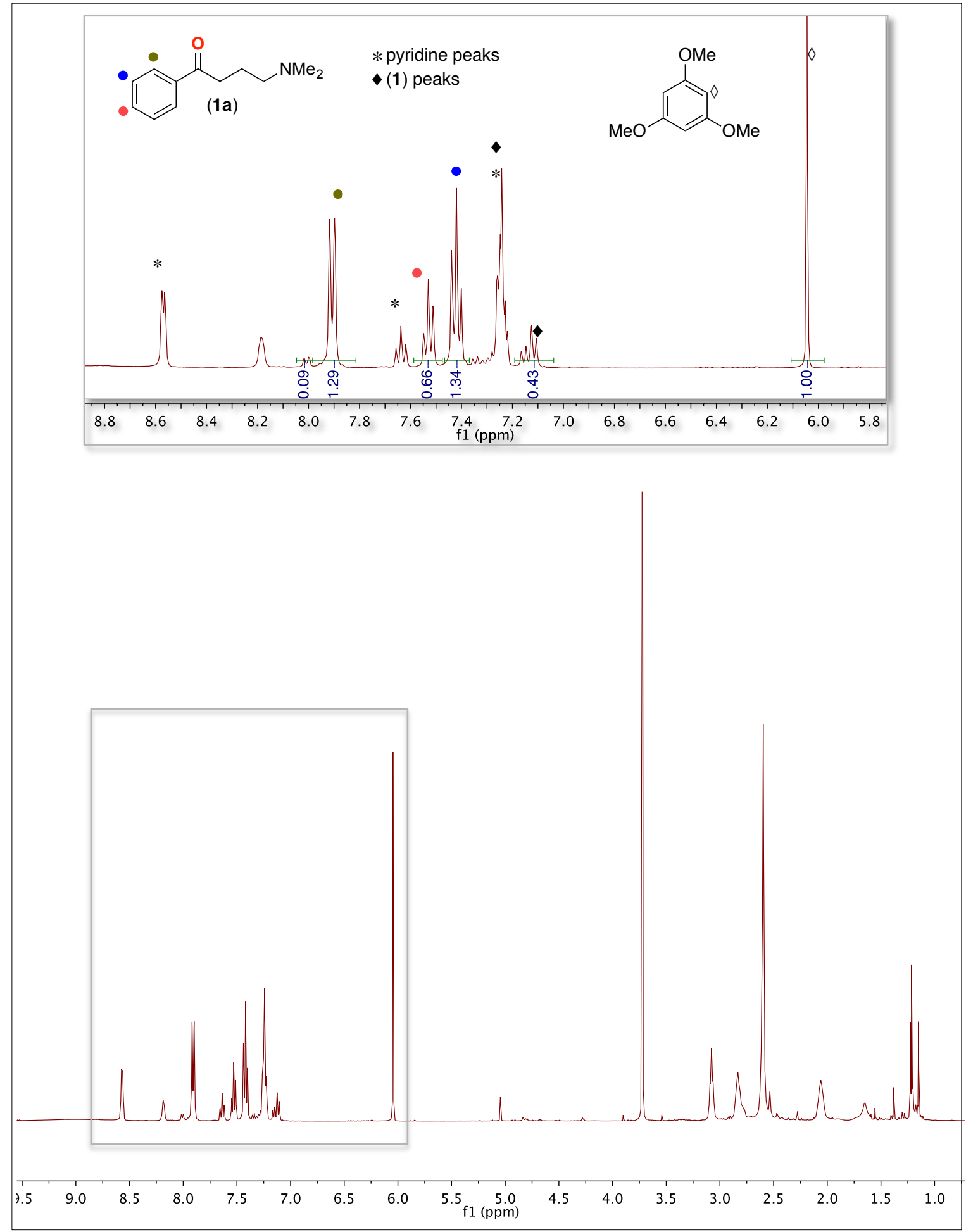

As shown in Table S1, we set out to optimize the oxidation reaction of amine 1. Catalyst screening revealed that all of the $\mathrm{Fe}(\mathrm{II})$ and $\mathrm{Fe}(\mathrm{III})$ catalysts investigated gave comparable yields of oxidation product 1a (between 18-24\% after $24 \mathrm{~h}$ with 3 equiv of ${ }^{t} \mathrm{BuOOH}$; entries $1-6$ ). We 
elected to proceed with inexpensive $\mathrm{FeCl}_{3}$ as the catalyst of choice. Replacing the oxidant with $\mathrm{H}_{2} \mathrm{O}_{2}$ resulted in only $8 \%$ yield (entry 7). Addition of more ${ }^{t} \mathrm{BuOOH}$ oxidant (6 equiv) via slow addition gave an increased yield of $38 \%$ (entry 8 ). Lowering the catalyst amount to 5 mol \% (entry 9) gave a similar yield. Further increasing the amount of oxidant to 12 equiv gave an improved yield of $60 \%$, but required a longer reaction time of $48 \mathrm{~h}$ (entry 10). As shown in entry 11, lowering the catalyst amount to $1 \mathrm{~mol} \%$ yielded $63 \%$ of product (1a) over $96 \mathrm{~h}$. Given the long reaction times and low cost of $\mathrm{FeCl}_{3}, 5 \mathrm{~mol} \%$ was chosen as the optimum catalyst loading.

Next we explored reaction solvents (entries 12-17). Replacing $\mathrm{MeCN}$ with $\mathrm{H}_{2} \mathrm{O}$ in entry 12 gave $61 \%$ yield over $72 \mathrm{~h}$. Eliminating pyridine from the reaction with $\mathrm{H}_{2} \mathrm{O}$ or $\mathrm{MeCN}$ led to negligible consumption of oxidant past $72 \mathrm{~h}$, and thus NMR yields were not determined (entries 13-14). However, using pyridine as the reaction solvent gave $44 \%$ yield of product 1a (entry 15 ). This is an interesting result which seems to suggest that even in the presence of excess pyridine, the amine nitrogen is sufficiently protonated to favor formation of 1a. Lowering the amount of pyridine in entry 16 by using a pyridine/MeCN (1:30) solvent mixture gave 44\% yield with 6 equiv of oxidant. To examine if oxidation was occurring at all with $\mathrm{MeCN}$ alone (entry 14), the oxidant amount was lowered to 1.5 equiv in entry 17; however, no reaction was observed after 24 h.

The role of picolinic acid was also examined by varying the loading from 0 to $25 \mathrm{~mol} \%$ (entries 18-20). Compared to entry 9, increasing the amount of picolinic acid two-fold to $25 \mathrm{~mol}$ $\%$ had no effect on the reaction giving $61 \%$ yield after $48 \mathrm{~h}$ (entry 18), and reducing it to $6.25 \mathrm{~mol}$ $\%$ gave $64 \%$ after an extended time of $72 \mathrm{~h}$ (entry 19). As shown in entry 20, eliminating 2picolinic acid from this reaction resulted in negligible consumption of oxidant past $72 \mathrm{~h}$ and thus NMR yields were not determined.

Compared to entry 9 , further increasing the amount of oxidant to 18 equiv gave a slightly increased yield of $65 \%$ (entry 21). An acid screen was also performed to assess the importance of acid strength and counterion effects (entries 22-28). Increasing the amount of $\mathrm{CF}_{3} \mathrm{CO}_{2} \mathrm{H}$ to $1.5,2.0$ and 3.0 equiv gave $63 \%, 58 \%$ and $51 \%$ yield, respectively (entries 22-24). Switching the acid additive to $\mathrm{HBF}_{4}$ (1.1 equiv), $\mathrm{HCl}$ (1.1 equiv) and $\mathrm{H}_{2} \mathrm{SO}_{4}$ (1.1 equiv) gave $61 \%, 51 \%$, and $40 \%$ yield, respectively, after $48 \mathrm{~h}$ (entries 25-27). Using $\mathrm{CH}_{3} \mathrm{CO}_{2} \mathrm{H}$ (1.1 equiv) resulted in only $24 \%$ yield of $\mathbf{1 a}$ and significant decomposition of $\mathbf{1}$ was observed (entry 28). 


\section{IId. Oxidation of 1 Under $\mathrm{Fe}(\mathrm{PDP}) / \mathrm{H}_{2} \mathrm{O}_{2}$ Catalysis $^{1}$}

To a $20 \mathrm{~mL}$ glass vial charged with a stir-bar and $N, N$-dimethyl phenylbutylamine (1) (44 $\mathrm{mg}, 0.25 \mathrm{mmol})$ was added $\mathrm{MeCN}(373 \mu \mathrm{L})$, and the solution was cooled to $0{ }^{\circ} \mathrm{C}$ in an ice/water bath. A solution of $\mathrm{HBF}_{4}\left(36 \mu \mathrm{L}, 48 \%\right.$ in $\left.\mathrm{H}_{2} \mathrm{O}\right)$ was added, and the solution was stirred at $0{ }^{\circ} \mathrm{C}$ for $10 \mathrm{~min}$ and at room temperature for $30 \mathrm{~min}$. A solution of $\mathrm{Fe}(\mathrm{PDP})(12 \mathrm{mg})$ and $\mathrm{AcOH}(7 \mu \mathrm{L})$ in MeCN $(250 \mu \mathrm{L})$ was added. Next, a solution of $\mathrm{H}_{2} \mathrm{O}_{2}\left(17 \mu \mathrm{L}, 50\right.$ wt \% in $\left.\mathrm{H}_{2} \mathrm{O}\right)$ in $\mathrm{MeCN}(2.25$ $\mathrm{mL}$ ) was added dropwise over at least $2 \mathrm{~min}$. After $10 \mathrm{~min}$ a second portion of $\mathrm{Fe}(\mathrm{PDP})$ and $\mathrm{AcOH}$ in $\mathrm{MeCN}$ was added, followed by the addition of a solution of $\mathrm{H}_{2} \mathrm{O}_{2}$ in $\mathrm{MeCN}$ over 2 min. After 10 min, a third portion of $\mathrm{Fe}(\mathrm{PDP})$ and $\mathrm{AcOH}$ in $\mathrm{MeCN}$ was added, followed by addition of a solution of $\mathrm{H}_{2} \mathrm{O}_{2}$ in $\mathrm{MeCN}$ over 2 min. After stirring for $24 \mathrm{~h}$, the reaction still tested positive for peroxide and was quenched with a saturated aqueous solution of $\mathrm{Na}_{2} \mathrm{~S}_{2} \mathrm{O}_{3}$ until the peroxide test was negative. The resulting mixture was subjected to the work-up procedure described in IIc for determining crude NMR yields.

As shown in Figure S2, no benzylic oxidation products were observed under these conditions; instead, it appears that decomposition of the substrate occurred to unidentifiable products.

Figure S2. ${ }^{1} \mathrm{H}$ NMR spectrum of the crude reaction mixture under $\mathrm{Fe}(\mathrm{PDP}) / \mathrm{H}_{2} \mathrm{O}_{2}$ catalysis

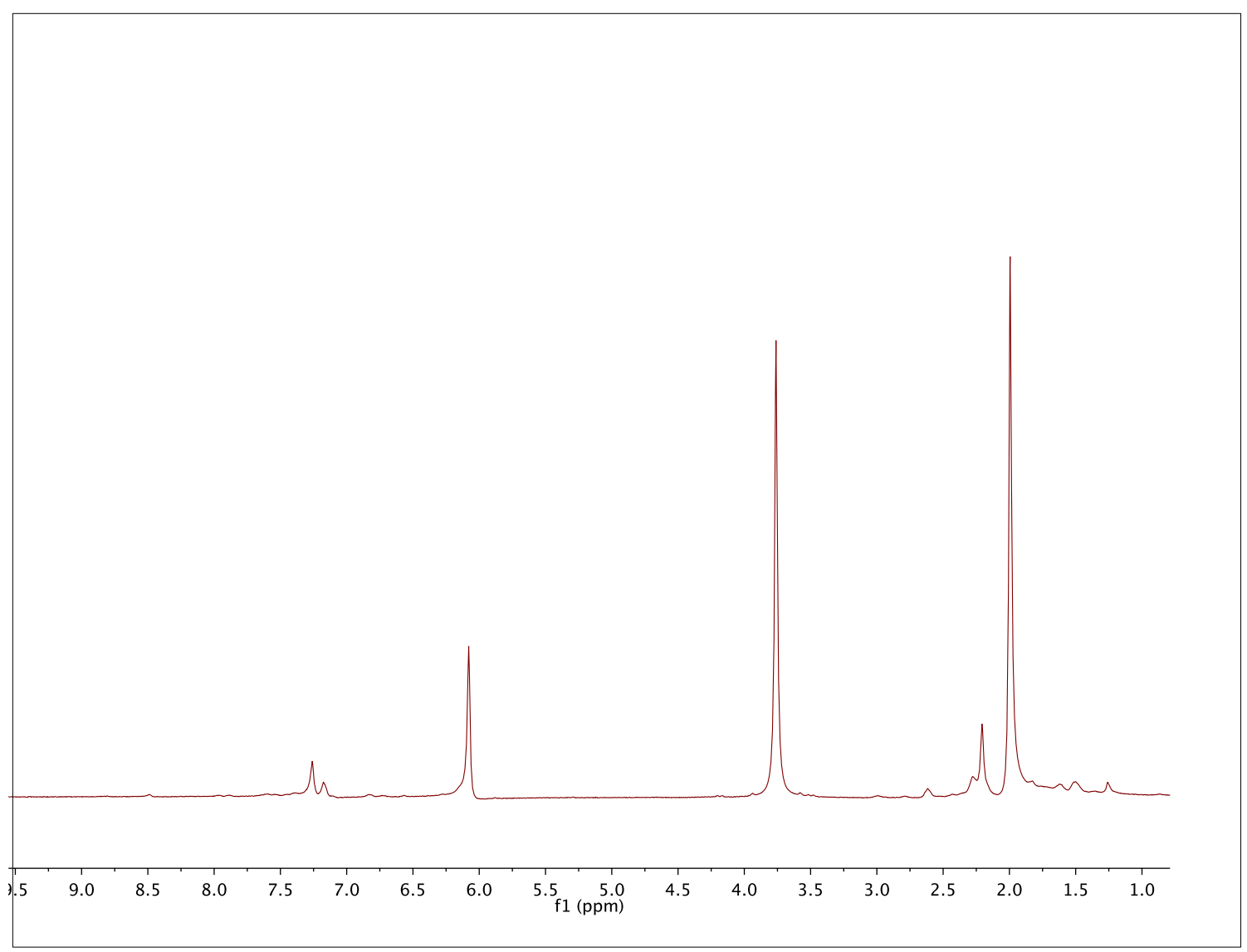




\section{General Procedure I for Amine Methylation}

This procedure was adapted from the method of Clarke and coworkers. ${ }^{2}$ A $25 \mathrm{~mL}$ round bottom flask containing the amine $(10 \mathrm{mmol})$ was cooled to $0{ }^{\circ} \mathrm{C}$ in an ice/water bath. Formic acid $(1.9 \mathrm{~mL}, 50 \mathrm{mmol})$ was added slowly, followed by formaldehyde (36 wt \%, $1.8 \mathrm{~mL}, 22 \mathrm{mmol}$ ). The reaction mixture was allowed to warm to room temperature, and then refluxed for $16 \mathrm{~h}$. After cooling to room temperature, $2 \mathrm{M}$ aqueous $\mathrm{HCl}(5 \mathrm{~mL})$ was added, and the mixture was concentrated to dryness. The resulting crude material was dissolved in $\mathrm{H}_{2} \mathrm{O}(5 \mathrm{~mL})$, basified with $20 \%$ aqueous $\mathrm{NaOH}(\mathrm{w} / \mathrm{v})$ and extracted into diethyl ether $(3 \times 10 \mathrm{~mL})$. The combined organic extracts were dried over $\mathrm{MgSO}_{4}$, filtered, and concentrated to furnish the methylated amine products.

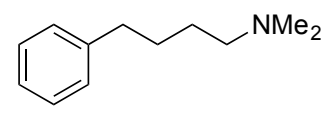

(1): Yellow oil (1.42 g, 80\% yield). ${ }^{1}$ H NMR (400 MHz, $\left.\mathrm{CDCl}_{3}\right) \delta 7.29-7.25$ (m, 2H), 7.20-7.15 (multiple peaks, 3H), 2.63 (t, $J=7.5 \mathrm{~Hz}, 2 \mathrm{H}), 2.27$ (t, $J=7.5 \mathrm{~Hz}, 2 \mathrm{H}$ ), 2.20 (s, $6 \mathrm{H}), 1.64(\mathrm{p}, J=7.7 \mathrm{~Hz}, 2 \mathrm{H}), 1.50(\mathrm{p}, J=7.7 \mathrm{~Hz}, 2 \mathrm{H}) .{ }^{13} \mathbf{C} \mathbf{N M R}\left(100 \mathrm{MHz}, \mathrm{CDCl}_{3}\right) \delta 142.6$, $128.5,128.3,125.7,59.8,45.6,36.0,29.4,27.5$. This compound has been previously reported. ${ }^{3}$

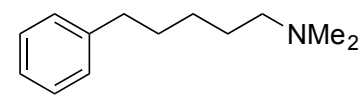

(2): Synthesized according to general procedure I on a $1.2 \mathrm{mmol}$ scale to give a yellow oil (176 mg, 77\% yield). ${ }^{1} \mathbf{H}$ NMR (400 MHz, $\left.\mathrm{CDCl}_{3}\right) \delta 7.32-7.23(\mathrm{~m}, 2 \mathrm{H}), 7.22-$ 7.16 (multiple peaks, 3H), 2.62 (t, $J=7.7 \mathrm{~Hz}, 2 \mathrm{H}$ ), 2.27-2.19 (multiple peaks, 8H), 1.65 (p, $J=7.7$ $\mathrm{Hz}, 2 \mathrm{H}), 1.49$ (p, $J=7.7 \mathrm{~Hz}, 2 \mathrm{H}), 1.36(\mathrm{p}, J=7.7 \mathrm{~Hz}, 2 \mathrm{H}) .{ }^{13} \mathbf{C} \mathbf{N M R}\left(100 \mathrm{MHz}, \mathrm{CDCl}_{3}\right) \delta 142.8$, $128.5,128.4,125.7,60.0,45.7,36.1,31.6,27.8,27.3$. HRMS (ESI) $\mathrm{m} / z$ calc for $\left[\mathrm{C}_{13} \mathrm{H}_{21} \mathrm{NH}\right]^{+}$ 192.1747, found 192.1740 .

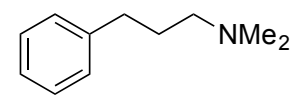

(3): Yellow oil (1.38 g, 85\% yield). ${ }^{1} \mathbf{H}$ NMR (400 MHz, $\left.\mathrm{CDCl}_{3}\right) \delta 7.30-7.25$ (m, 2H), 7.21-7.16 (multiple peaks, 3H), 2.67-2.62 (m, 2H), 2.36-2.28 (m, 2H), $2.24(\mathrm{~s}, 6 \mathrm{H}), 1.80$ $(\mathrm{p}, J=7.6 \mathrm{~Hz}, 2 \mathrm{H}) .{ }^{13} \mathbf{C}$ NMR $\left(100 \mathrm{MHz}, \mathrm{CDCl}_{3}\right) \delta 142.4,128.5,128.4,125.8,59.4,45.6,33.8$, 29.6. This compound has been previously reported. ${ }^{3}$

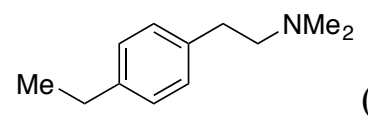

(6): Yellow oil (1.39 g, 79\% yield). ${ }^{1} \mathbf{H}$ NMR (400 $\left.\mathrm{MHz} \mathrm{CDCl}_{3}\right) \delta 7.12$ (app. s, 4H), 2.80-2.70 (m, 2H), 2.62 (q, $J=7.6 \mathrm{~Hz}, 2 \mathrm{H}), 2.55-2.48(\mathrm{~m}, 2 \mathrm{H}), 2.29$ (s, 6H), 1.22 (t, $J$ 
$=7.6 \mathrm{~Hz}, 3 \mathrm{H}) .{ }^{13} \mathbf{C}$ NMR $\left(100 \mathrm{MHz}, \mathrm{CDCl}_{3}\right) \delta 142.0,137.6,128.7,128.0,61.8,45.6,34.1,28.6$, 15.7. HRMS (ESI) $\mathrm{m} / z$ calc'd for $\left[\mathrm{C}_{12} \mathrm{H}_{19} \mathrm{NH}\right]^{+} 178.1590$, found 178.1585 .

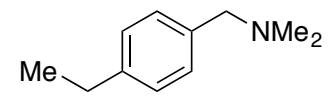

(7): Clear oil (1.31 g, 80\% yield). ${ }^{1}$ H NMR (400 MHz, $\left.\mathrm{CDCl}_{3}\right) \delta 7.21(\mathrm{~d}, J=$ $8.0 \mathrm{~Hz}, 2 \mathrm{H}), 7.15$ (d, $J=8.0 \mathrm{~Hz}, 2 \mathrm{H}), 3.39$ (s, 2H), 2.64 (q, $J=7.6 \mathrm{~Hz}, 2 \mathrm{H}), 2.23$ (s, 6H), 1.23 (t, $J$ $=7.6 \mathrm{~Hz}, 3 \mathrm{H}) .{ }^{13} \mathbf{C}$ NMR $\left(100 \mathrm{MHz}, \mathrm{CDCl}_{3}\right) \delta 143.1,136.2,129.2,127.8,64.3,45.5,28.6,15.7$. HRMS (ESI) $m / z$ calc for $\left[\mathrm{C}_{11} \mathrm{H}_{17} \mathrm{NH}\right]^{+} 164.1434$, found 164.1427 .

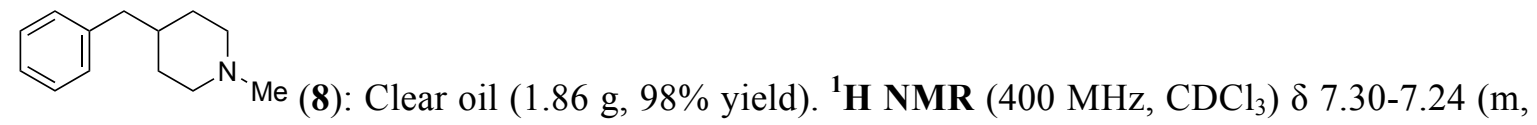
2H), 7.21-7.11 (multiple peaks, 3H), 2.89-2.75 (m, 2H), 2.53 (d, $J=7.1 \mathrm{~Hz}, 2 \mathrm{H}), 2.24$ (s, 3H), $1.84(\mathrm{td}, J=11.8,2.5 \mathrm{~Hz}, 2 \mathrm{H}), 1.67-1.59(\mathrm{~m}, 2 \mathrm{H}), 1.55-1.43(\mathrm{~m}, 1 \mathrm{H}), 1.37-1.25(\mathrm{~m}, 2 \mathrm{H}) .{ }^{13} \mathbf{C}$ NMR $\left(100 \mathrm{MHz}, \mathrm{CDCl}_{3}\right) \delta 140.8,129.2,128.2,125.8,56.1,46.6,43.3,37.5,32.4$. This compound has been previously reported. ${ }^{4}$

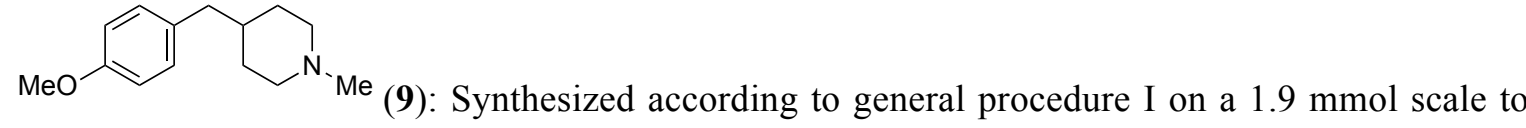
give a clear oil (323 mg, 59\% yield). ${ }^{1} \mathbf{H}$ NMR (400 MHz, $\left.\mathrm{CDCl}_{3}\right) \delta 7.05(\mathrm{~d}, J=8.6 \mathrm{~Hz}, 2 \mathrm{H}), 6.82$ (d, $J=8.6 \mathrm{~Hz}, 2 \mathrm{H}), 3.78$ (s, 3H), 2.81 (br. d, $J=11.7 \mathrm{~Hz}, 2 \mathrm{H}), 2.47$ (d, $J=7.0 \mathrm{~Hz}, 2 \mathrm{H}), 2.24$ (s, $3 \mathrm{H}), 1.84(\mathrm{td}, J=11.7,2.2 \mathrm{~Hz}, 2 \mathrm{H}), 1.67-1.58(\mathrm{~m}, 2 \mathrm{H}), 1.50-1.38(\mathrm{~m}, 1 \mathrm{H}), 1.34-1.23(\mathrm{~m}, 2 \mathrm{H}) .{ }^{13} \mathbf{C}$ NMR $\left(100 \mathrm{MHz}, \mathrm{CDCl}_{3}\right) \delta 157.8,132.9,130.1,113.7,56.1,55.3,46.6,42.4,37.6,32.4$. HRMS (ESI) $m / z$ calc for $\left[\mathrm{C}_{14} \mathrm{H}_{21} \mathrm{NOH}\right]^{+} 220.1696$, found 220.1691 .

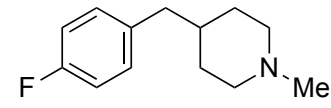

(10): Synthesized according to general procedure I on a $5.0 \mathrm{mmol}$ scale to give a clear oil (505 mg, 49\% yield). ${ }^{1} \mathbf{H}$ NMR (400 MHz, $\left.\mathrm{CDCl}_{3}\right) \delta$ 7.16-7.02 (m, 2H), 6.99-6.91 (m, 2H), 2.81 (br. d, $J=11.8 \mathrm{~Hz}, 2 \mathrm{H}), 2.50$ (d, $J=7.0 \mathrm{~Hz}, 2 \mathrm{H}), 2.24$ (s, 3H), 1.84 (td, $J=11.8,2.2$ $\mathrm{Hz}, 2 \mathrm{H}), 1.64-1.58(\mathrm{~m}, 2 \mathrm{H}), 1.51-1.38(\mathrm{~m}, 1 \mathrm{H}), 1.34-1.22(\mathrm{~m}, 2 \mathrm{H}) .{ }^{13} \mathbf{C}$ NMR $\left(100 \mathrm{MHz}, \mathrm{CDCl}_{3}\right) \delta$ $161.4\left(\mathrm{~d}, J_{\mathrm{C}-\mathrm{F}}=243.2 \mathrm{~Hz}\right), 136.4\left(\mathrm{~d}, J_{\mathrm{C}-\mathrm{F}}=3.3 \mathrm{~Hz}\right), 130.5\left(\mathrm{~d}, J_{\mathrm{C}-\mathrm{F}}=7.7 \mathrm{~Hz}\right), 115.0\left(\mathrm{~d}, J_{\mathrm{C}-\mathrm{F}}=21.0\right.$ $\mathrm{Hz}), 56.0,46.6,42.5,37.5,32.3 .{ }^{19} \mathbf{F}$ NMR $\left(377 \mathrm{MHz}, \mathrm{CDCl}_{3}\right) \delta-117.9$. HRMS (ESI) $\mathrm{m} / \mathrm{z}$ calc for $\left[\mathrm{C}_{13} \mathrm{H}_{18} \mathrm{FNH}\right]^{+}$208.1496, found 208.1491 


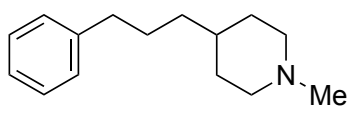

(11): Synthesized according to general procedure I on a $5.0 \mathrm{mmol}$ scale to give a clear oil (1.0 g, 92\% yield). ${ }^{1} \mathbf{H}$ NMR (400 MHz, $\left.\mathrm{CDCl}_{3}\right) \delta$ 7.31-7.23 (m, 2H), 7.20-7.15 (multiple peaks, 3H), 2.82 (br. d, $J=11.6 \mathrm{~Hz}, 2 \mathrm{H}), 2.59$ (t, $J=7.8 \mathrm{~Hz}, 2 \mathrm{H}), 2.24$ (s, 3H), 1.94-1.80 (m, 2H), 1.72-1.58 (multiple peaks, 4H), 1.33-1.17 (multiple peaks, 5H). ${ }^{13} \mathbf{C}$ NMR (100 MHz, $\left.\mathrm{CDCl}_{3}\right) \delta 142.8,128.5,128.3,125.7,56.2,46.7,36.4,36.3,35.2,32.6,28.9$. HRMS (ESI) $\mathrm{m} / z$ calc for $\left[\mathrm{C}_{15} \mathrm{H}_{23} \mathrm{NH}\right]^{+} 218.1903$, found 218.1900 .

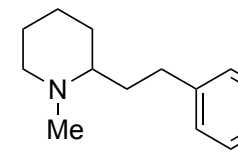

(23): Synthesized according to general procedure I on a $2.5 \mathrm{mmol}$ scale to give a clear oil (347 mg, 68\% yield). ${ }^{1} \mathbf{H}$ NMR (400 MHz, $\left.\mathrm{CDCl}_{3}\right) \delta$ 7.31-7.26 (m, 2H), 7.21-7.16 (multiple peaks, 3H), $2.86(\mathrm{dtd}, J=11.6,3.7,1.5 \mathrm{~Hz}, 1 \mathrm{H}), 2.76-2.68(\mathrm{~m}, 1 \mathrm{H}), 2.63-2.50(\mathrm{~m}, 1 \mathrm{H})$, $2.28(\mathrm{~s}, 3 \mathrm{H}), 2.12-2.04(\mathrm{~m}, 1 \mathrm{H}), 1.97-1.84$ (multiple peaks, 2H), 1.79-1.69 (multiple peaks, 3H), 1.63-1.56 (m, 2H), 1.47-1.36 (m, 1H), 1.35-1.22 (m, 1H). $\left.{ }^{13} \mathbf{C ~ N M R ~ ( 1 0 0 ~ M H z , ~} \mathrm{CDCl}_{3}\right) \delta 143.0$, 128.5, 128.4, 125.8, 63.6, 57.4, 43.2, 34.9, 31.4, 30.9, 26.1, 24.6. HRMS (ESI) $\mathrm{m} / z$ calc for $\left[\mathrm{C}_{14} \mathrm{H}_{21} \mathrm{~N}\right]^{+}$204.1747, found 204.1742. 


\section{General Procedure II for $\boldsymbol{N}$-Alkylation of Amines}

To a $50 \mathrm{~mL}$ round bottom flask charged with a stir bar were sequentially added amine (5.0 $\mathrm{mmol})$, acetonitrile $(10 \mathrm{~mL}), \mathrm{K}_{2} \mathrm{CO}_{3}(898 \mathrm{mg}, 6.5 \mathrm{mmol})$, $\mathrm{KI}(83 \mathrm{mg}, 0.50 \mathrm{mmol})$, and alkyl bromide $(5.0 \mathrm{mmol})$. The resulting mixture was heated at reflux for approximately $18 \mathrm{~h}$. The reaction mixture was allowed to cool to room temperature. $\mathrm{K}_{2} \mathrm{CO}_{3}$ was removed by filtration, and the filtrate was concentrated under vacuum. The crude material was purified via automated column chromatography on a Biotage instrument using a $25 \mathrm{~g}$ Ultra SNAP normal phase cartridge, eluting with a $\mathrm{Et}_{3} \mathrm{~N} / \mathrm{EtOAc} /$ hexanes solvent mixture to furnish the desired product.

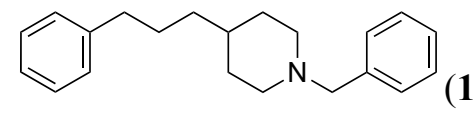

(12): Eluted with $50 \%$ of $\mathrm{Et}_{3} \mathrm{~N} / \mathrm{EtOAc} /$ hexanes $(1: 10: 10)$ in hexanes to give a yellow oil (715 mg, 49\% yield). ${ }^{1} \mathbf{H}$ NMR (400 $\left.\mathrm{MHz}, \mathrm{CDCl}_{3}\right) \delta$ 7.33-7.23 (multiple peaks, 7H), 7.21-7.16 (multiple peaks, 3H), 3.49 (s, 2H), 2.87 (br. d, $J=10.8 \mathrm{~Hz}, 2 \mathrm{H}), 2.65-2.55$ (m, 2H), 1.97-1.86 (m, 2H), 1.69-1.58 (multiple peaks, 4H), 1.33-1.20 (multiple peaks, 5H). ${ }^{13} \mathbf{C}$ NMR (100 $\left.\mathrm{MHz}, \mathrm{CDCl}_{3}\right) \delta 142.9,138.8,129.3,128.5,128.4,128.2,127.0,125.7,63.7,54.1,36.4,36.3,35.8$, 32.6, 28.9. HRMS (ESI) $m / z$ calc for $\left[\mathrm{C}_{21} \mathrm{H}_{27} \mathrm{NH}\right]^{+} 294.2216$, found 294.2213.

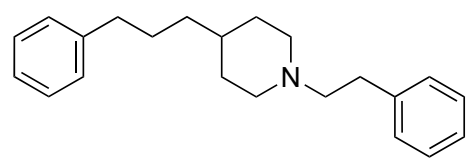

(13): Purified by extraction. Crude material was dissolved in EtOAc $(10 \mathrm{~mL})$ and basified with $0.5 \mathrm{M}$ aqueous $\mathrm{NaOH}$. The organic layer was collected, and the aqueous layer was extracted with EtOAc $(10 \mathrm{~mL})$. The combined organic layers were back-extracted with a saturated aqueous $\mathrm{NaCl}$ solution $(10 \mathrm{~mL})$, dried over $\mathrm{MgSO}_{4}$, filtered, and concentrated to give the desired product as a yellow oil (587 $\mathrm{mg}, 38 \%$ yield). ${ }^{1} \mathbf{H}$ NMR $\left(400 \mathrm{MHz}, \mathrm{CDCl}_{3}\right) \delta$ 7.32-7.27 (multiple peaks, 4H), 7.23-7.16 (multiple peaks, 6H), 3.00 (br. d, $J=10.8 \mathrm{~Hz}, 2 \mathrm{H}$ ), 2.85-2.80 (m, 2H), 2.64-2.55 (multiple peaks, 4H), 2.03-1.93 (m, 2H), 1.75-1.60 (multiple peaks, 4H), 1.36-1.22 (multiple peaks, 5H). ${ }^{13} \mathbf{C}$ NMR (100 MHz, $\left.\mathrm{CDCl}_{3}\right) \delta 142.9,140.8,128.8,128.5,128.5,128.4$, $126.1,125.7,61.3,54.2,36.4,36.3,35.9,34.0,32.6,28.9$. HRMS (ESI) $m / z$ calc for $\left[\mathrm{C}_{22} \mathrm{H}_{29} \mathrm{NH}\right]^{+}$ 308.2373, found 308.2369.

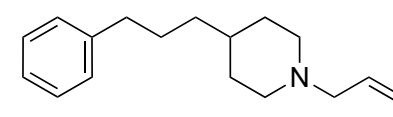

(14): Eluted with $50 \%$ of $\mathrm{Et}_{3} \mathrm{~N} / \mathrm{EtOAc} /$ hexanes $(1: 10: 10)$ in hexanes to give a clear oil (447 mg, 37\% yield). ${ }^{1} \mathbf{H}$ NMR (400 MHz, $\left.\mathrm{CDCl}_{3}\right) \delta$ 7.32-7.25 (m, 2H), 7.21-7.15 (multiple peaks, 3H), 5.89 (ddt, $J=16.9,10.2,6.6 \mathrm{~Hz}, 1 \mathrm{H}$ ), 5.21-5.08 (multiple peaks, 2H), 2.97 
(d, $J=6.6 \mathrm{~Hz}, 2 \mathrm{H}), 2.91$ (br. d, $J=10.8 \mathrm{~Hz}, 2 \mathrm{H}), 2.59$ (t, $J=7.8 \mathrm{~Hz}, 2 \mathrm{H}), 1.93-1.81$ (m, 2H), 1.721.58 (multiple peaks, $4 \mathrm{H}), 1.35-1.16$ (multiple peaks, $5 \mathrm{H}) .{ }^{13} \mathbf{C ~ N M R}\left(100 \mathrm{MHz}, \mathrm{CDCl}_{3}\right) \delta 142.9$, 135.8, 128.5, 128.3, 125.7, 117.6, 62.4, 54.1, 36.4, 36.3, 35.7, 32.5, 28.9. HRMS (ESI) $\mathrm{m} / z$ calc for $\left[\mathrm{C}_{17} \mathrm{H}_{25} \mathrm{NH}\right]^{+} 244.2060$, found 244.2055.

(15): Eluted with 50\% of Et ${ }_{3} \mathrm{~N} /$ EtOAc/hexanes $(1: 10: 10)$ in hexanes to give a slightly yellowish oil (757 mg, 56\% yield). ${ }^{1} \mathbf{H}$ NMR $\left(400 \mathrm{MHz}, \mathrm{CDCl}_{3}\right) \delta 7.32-7.23(\mathrm{~m}$, 2H), 7.20-7.14 (multiple peaks, 3H), 2.83 (br. d, $J=11.7 \mathrm{~Hz}, 2 \mathrm{H}), 2.64-2.56(\mathrm{~m}, 2 \mathrm{H}), 2.40$ (multiple peaks, t, $J=7.1 \mathrm{~Hz}, 4 \mathrm{H}), 1.96-1.85(\mathrm{~m}, 2 \mathrm{H}), 1.81(\mathrm{p}, J=7.1 \mathrm{~Hz}, 2 \mathrm{H}), 1.70-1.57$ (multiple peaks, 4H), 1.31-1.12 (multiple peaks, 5H). ${ }^{13} \mathbf{C}$ NMR $\left(100 \mathrm{MHz}, \mathrm{CDCl}_{3}\right) \delta 142.8,128.4$, $128.3,125.7,120.0,56.9,54.1,36.3,36.2,35.8,32.5,28.8,23.1,15.1$. HRMS (ESI) $\mathrm{m} / z$ calc for $\left[\mathrm{C}_{18} \mathrm{H}_{26} \mathrm{~N}_{2} \mathrm{H}\right]^{+}$271.2169, found 271.2167.

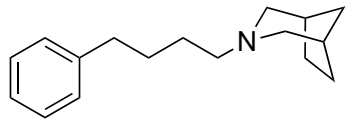

(20): Synthesized on a $1.8 \mathrm{mmol}$ scale and eluted with $50 \%$ of Et $_{3} \mathrm{~N} /$ EtOAc/hexanes $(1: 10: 10)$ in hexanes to give a yellow oil (407 $\mathrm{mg}, 93 \%$ yield). ${ }^{1} \mathbf{H}$ NMR $\left(500 \mathrm{MHz}, \mathrm{CDCl}_{3}\right) \delta$ 7.30-7.27 (m, 2H), 7.21-7.14 (multiple peaks, 3H), 2.67-2.59 (multiple peaks, 4H), 2.27 (t, $J=7.3 \mathrm{~Hz}, 2 \mathrm{H}), 2.08$ (br. s, 2H), 1.94 (d, $J=10.2 \mathrm{~Hz}, 2 \mathrm{H}$ ), 1.68-1.60 (multiple peaks, 4H), 1.56-1.41 (multiple peaks, 5H), 1.35-1.28 (m, 1H). ${ }^{13} \mathbf{C}$ NMR $\left(125 \mathrm{MHz}, \mathrm{CDCl}_{3}\right) \delta$ $143.1,128.5,128.4,125.7,60.6,58.1,37.9,36.0,35.4,29.4,28.9,26.8$. HRMS (ESI) $\mathrm{m} / z$ calc for $\left[\mathrm{C}_{17} \mathrm{H}_{25} \mathrm{NH}\right]^{+}$244.2060, found 244.2059

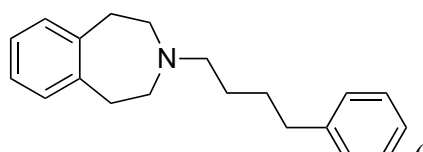

(21): Synthesized on a $3.4 \mathrm{mmol}$ scale and was eluted with $25 \%$ of $\mathrm{Et}_{3} \mathrm{~N} /$ EtOAc/hexanes (1:10:10) in hexanes to give a brownish oil (388 $\mathrm{mg}, 41 \%$ yield). ${ }^{1} \mathbf{H}$ NMR $\left(400 \mathrm{MHz}, \mathrm{CDCl}_{3}\right) \delta$ 7.33-7.27 (m, 2H), 7.23-7.17 (multiple peaks, 3H), 7.15-7.08 (multiple peaks, 4H), 2.96-2.90 (m, 4H), 2.71-2.61 (multiple peaks, 6H), 2.56-2.47 (m, 2H), 1.78-1.51 (multiple peaks, 4H). ${ }^{13} \mathrm{C}$ NMR $\left(100 \mathrm{MHz}, \mathrm{CDCl}_{3}\right) \delta 142.6,142.4,129.0,128.5,128.4,126.3$, 125.8, 59.2, 55.5, 36.8, 36.0, 29.6, 26.7. HRMS (ESI) $\mathrm{m} / z$ calc for $\left[\mathrm{C}_{20} \mathrm{H}_{25} \mathrm{NH}\right]^{+} 280.2060$, found 280.2055 . 


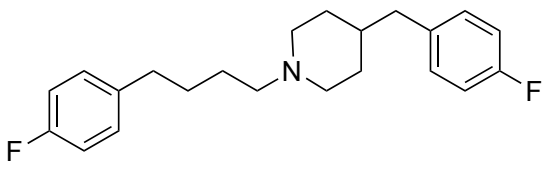

(24): Synthesized on a $2.5 \mathrm{mmol}$ scale and eluted with a 10 $50 \%$ gradient of $\mathrm{Et}_{3} \mathrm{~N} / \mathrm{EtOAc} /$ hexanes $(1: 10: 10)$ in hexanes to give a yellow oil $(352 \mathrm{mg}, 41 \%$ yield). ${ }^{1} \mathbf{H}$ NMR (400 MHz, $\mathrm{CDCl}_{3}$ ) $\delta$ 7.16-7.05 (multiple peaks, $4 \mathrm{H}$ ), 7.01-6.91 (multiple peaks, 4H), 2.88 (br. d, $11.5 \mathrm{~Hz}, 2 \mathrm{H}), 2.64-2.55$ (m, 2H), 2.51 (d, $J=7.0 \mathrm{~Hz}, 2 \mathrm{H}), 2.33-2.27$ (m, 2H), 1.89-1.78 (m, 2H), 1.65-1.56 (multiple peaks, 4H), 1.56-1.42 (multiple peaks, 3H), 1.36-1.23 (m, 2H). ${ }^{13}$ C NMR $\left(100 \mathrm{MHz}, \mathrm{CDCl}_{3}\right) \delta 161.4\left(\mathrm{~d}, J_{\mathrm{C}-\mathrm{F}}=243.3 \mathrm{~Hz}\right), 161.3\left(\mathrm{~d}, J_{\mathrm{C}-\mathrm{F}}=243.0 \mathrm{~Hz}\right), 138.2$ $\left(\mathrm{d}, J_{\mathrm{C}-\mathrm{F}}=3.1 \mathrm{~Hz}\right), 136.4\left(\mathrm{~d}, J_{\mathrm{C}-\mathrm{F}}=3.2 \mathrm{~Hz}\right), 130.5\left(\mathrm{~d}, J_{\mathrm{C}-\mathrm{F}}=7.7 \mathrm{~Hz}\right), 129.8\left(\mathrm{~d}, J_{\mathrm{C}-\mathrm{F}}=7.7 \mathrm{~Hz}\right), 115.1$ $\left(\mathrm{d}, J_{\mathrm{C}-\mathrm{F}}=21.1 \mathrm{~Hz}\right), 115.0\left(\mathrm{~d}, J_{\mathrm{C}-\mathrm{F}}=21.0 \mathrm{~Hz}\right), 59.1,54.1,42.5,38.2,35.2,32.3,29.8,26.8$. HRMS (ESI) $m / z$ calc for $\left[\mathrm{C}_{22} \mathrm{H}_{27} \mathrm{~F}_{2} \mathrm{NH}\right]^{+} 344.2184$, found 344.2181 .

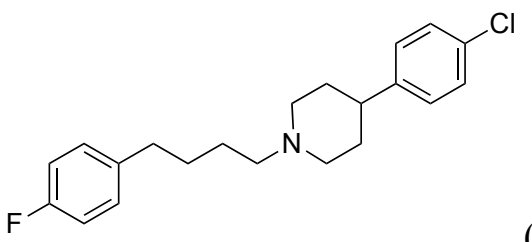

(26): Synthesized on a $2.5 \mathrm{mmol}$ scale, and eluted with a 10 $50 \%$ gradient of $\mathrm{Et}_{3} \mathrm{~N} / \mathrm{EtOAc} / \mathrm{hexanes}(1: 10: 10)$ in hexanes to give a white solid $(534 \mathrm{mg}, 62 \%$ yield). ${ }^{1} \mathbf{H}$ NMR $\left(400 \mathrm{MHz}, \mathrm{CDCl}_{3}\right) \delta$ 7.27-7.24 (m, 2H), 7.17-7.11 (multiple peaks, 4H), 6.996.92 (m, 2H), 3.02 (br. d, $J=11.6 \mathrm{~Hz}, 2 \mathrm{H}), 2.61$ (t, $J=7.3 \mathrm{~Hz}, 2 \mathrm{H}), 2.53-2.42$ (m, 1H), 2.41-2.34 (m, 2H), $2.00(\mathrm{td}, J=11.5,2.9 \mathrm{~Hz}, 2 \mathrm{H}), 1.85-1.71$ (multiple peaks, $4 \mathrm{H}), 1.68-1.50$ (multiple peaks, 4H). ${ }^{13}$ C NMR $\left(100 \mathrm{MHz}, \mathrm{CDCl}_{3}\right) \delta 161.3\left(\mathrm{~d}, J_{\mathrm{C}-\mathrm{F}}=242.9 \mathrm{~Hz}\right), 145.0,138.2\left(\mathrm{~d}, J_{\mathrm{C}-\mathrm{F}}=3.3 \mathrm{~Hz}\right)$, $131.8,129.8\left(\mathrm{~d}, J_{\mathrm{C}-\mathrm{F}}=7.7 \mathrm{~Hz}\right), 128.6,128.3,115.1\left(\mathrm{~d}, J_{\mathrm{C}-\mathrm{F}}=21.0 \mathrm{~Hz}\right), 59.1,54.5,42.3,35.2,33.6$, 29.8, 26.8. HRMS (ESI) $\mathrm{m} / z$ calc for $\left[\mathrm{C}_{21} \mathrm{H}_{25} \mathrm{ClFNH}\right]^{+} 346.1732$, found 346.1727 .

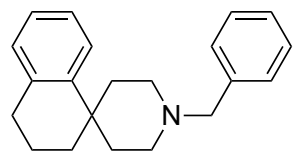

(27): Synthesized on a $4.4 \mathrm{mmol}$ scale and eluted with $25 \%$ of $\mathrm{Et}_{3} \mathrm{~N} / \mathrm{EtOAc} / \mathrm{hexanes}(1: 10: 10)$ in hexanes to give a white solid (806 mg, $63 \%$ yield). ${ }^{1} \mathbf{H}$ NMR $\left(400 \mathrm{MHz}, \mathrm{CDCl}_{3}\right) \delta 7.51$ (d, $\left.J=8.0 \mathrm{~Hz}, 1 \mathrm{H}\right), 7.40-7.31$ (multiple peaks, 4H), 7.31-7.22 (m, 1H), 7.21-7.15 (m, 1H), 7.11-7.02 (m, 2H), $3.58(\mathrm{~s}, 2 \mathrm{H}), 2.81-2.70(\mathrm{~m}, 4 \mathrm{H}), 2.29(\mathrm{td}, J=12.4,2.3 \mathrm{~Hz}$, $2 \mathrm{H}), 2.14(\mathrm{td}, J=13.2,4.2 \mathrm{~Hz}, 2 \mathrm{H}), 1.89-1.79(\mathrm{~m}, 2 \mathrm{H}), 1.79-1.65(\mathrm{~m}, 2 \mathrm{H}), 1.64-1.52(\mathrm{~m}, 2 \mathrm{H}) .{ }^{13} \mathbf{C}$ NMR $\left(100 \mathrm{MHz}, \mathrm{CDCl}_{3}\right) \delta 145.4,138.6,137.4,129.2,129.0,128.1,127.0,126.9,125.9,125.3$, 63.6, 49.6, 38.5, 35.4, 30.9, 30.8, 19.0. HRMS (ESI) $\mathrm{m} / z$ calc for $\left[\mathrm{C}_{21} \mathrm{H}_{25} \mathrm{NH}\right]^{+}$292.2060, found 292.2053. 


\section{Procedures for $N$-Alkylation of Amines 18, 19, and 22}

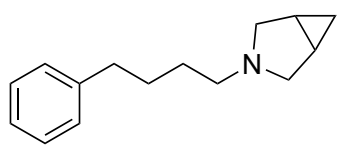

(18): A $20 \mathrm{~mL}$ vial was charged with 3-azabicyclo[3.1.0]hexane hydrochloride (359 mg, $3.00 \mathrm{mmol}$ ), $\mathrm{K}_{2} \mathrm{CO}_{3}$ (954 mg, $\left.6.90 \mathrm{mmol}\right), \mathrm{KI}$ (50 mg, $0.30 \mathrm{mmol}$ ), MeCN $(3 \mathrm{~mL})$, and a stir bar. To this mixture, 1-bromo-4-phenylbutane $(639 \mathrm{mg}, 3.00 \mathrm{mmol})$ was added as a solution in $\mathrm{MeCN}(3 \mathrm{~mL})$. The vial was sealed with a Teflon cap and heated at $90{ }^{\circ} \mathrm{C}$ for $18 \mathrm{~h}$. The reaction mixture was cooled to room temperature, filtered, and concentrated in vacuo. Purification by flash chromatography (elution gradient from hexanes to $5 \% \mathrm{NEt}_{3} / 10 \%$ EtOAc in hexanes) gave the title compound as a colorless oil (134 mg, 21\% yield). ${ }^{1} \mathbf{H}$ NMR (500 MHz, $\left.\mathrm{CDCl}_{3}\right) \delta 7.27(\mathrm{t}, J=7.6 \mathrm{~Hz}, 2 \mathrm{H}), 7.20-7.14$ (multiple peaks, 3H), $2.98(\mathrm{~d}, J=8.6 \mathrm{~Hz}, 2 \mathrm{H}), 2.60(\mathrm{t}$, $J=7.7 \mathrm{~Hz}, 2 \mathrm{H}), 2.40(\mathrm{t}, J=7.5 \mathrm{~Hz}, 2 \mathrm{H}), 2.28-2.22(\mathrm{~m}, 2 \mathrm{H}), 1.62(\mathrm{p}, J=7.7 \mathrm{~Hz}, 2 \mathrm{H}), 1.47(\mathrm{p}, J=$ $7.6 \mathrm{~Hz}, 2 \mathrm{H}), 1.33-1.29(\mathrm{~m}, 2 \mathrm{H}), 0.68(\mathrm{q}, J=3.8 \mathrm{~Hz}, 1 \mathrm{H}), 0.33(\mathrm{td}, J=7.7,4.2 \mathrm{~Hz}, 1 \mathrm{H}) .{ }^{13} \mathbf{C} \mathbf{~ N M R}$ $\left(125 \mathrm{MHz}, \mathrm{CDCl}_{3}\right) \delta 142.8,128.5,128.4,125.7,55.6,55.3,36.0,29.5,28.7,15.5,7.1$. HRMS (ESI) $m / z$ calc for $\left[\mathrm{C}_{15} \mathrm{H}_{21} \mathrm{NH}\right]^{+} 216.1747$, found: 216.1746 .

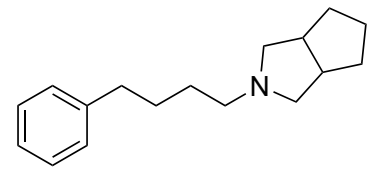

(19): A $20 \mathrm{~mL}$ vial was charged with cis-octahydrocyclopenta[c]pyrrole hydrochloride (443 mg, $3.00 \mathrm{mmol}), \mathrm{K}_{2} \mathrm{CO}_{3}(1.24 \mathrm{~g}, 9.00 \mathrm{mmol}), \mathrm{KI}(50 \mathrm{mg}, 0.30 \mathrm{mmol}), \mathrm{MeCN}$ $(3 \mathrm{~mL})$, and a stir bar. To this mixture, 1-bromo-4-phenylbutane $(639 \mathrm{mg}, 3.00 \mathrm{mmol})$ was added as a solution in $\mathrm{MeCN}(3 \mathrm{~mL})$. The vial was sealed with a Teflon cap and heated at $90{ }^{\circ} \mathrm{C}$ for $24 \mathrm{~h}$. The reaction mixture was cooled to room temperature, filtered, and concentrated in vacuo. Purification by flash chromatography (elution gradient from hexanes to $2 \% \mathrm{NEt}_{3} / 10 \%$ EtOAc in hexanes) gave the title compound as a pale yellow oil (539 mg, 74\% yield). ${ }^{1} \mathbf{H}$ NMR (500 MHz, $\left.\mathrm{CDCl}_{3}\right) \delta 7.29-7.24(\mathrm{~m}, 2 \mathrm{H}), 7.19-7.14$ (multiple peaks, 3H), $2.77(\mathrm{t}, J=8.4 \mathrm{~Hz}, 2 \mathrm{H}), 2.62(\mathrm{t}, J=$ $7.7 \mathrm{~Hz}, 2 \mathrm{H}), 2.60-2.54(\mathrm{~m}, 2 \mathrm{H}), 2.35(\mathrm{t}, J=7.7 \mathrm{~Hz}, 2 \mathrm{H}), 1.95(\mathrm{dd}, J=9.2,5.5 \mathrm{~Hz}, 2 \mathrm{H}), 1.68-1.35$ (multiple peaks, $10 \mathrm{H}) .{ }^{13} \mathbf{C}$ NMR $\left(125 \mathrm{MHz}, \mathrm{CDCl}_{3}\right) \delta 142.8,128.5,128.4,125.7,62.0,56.3,42.5$, 36.0, 32.68, 29.7, 28.7, 25.9. HRMS (ESI) $\mathrm{m} / z$ calc for $\left[\mathrm{C}_{17} \mathrm{H}_{25} \mathrm{NH}\right]^{+} 244.2060$, found 244.2058. 


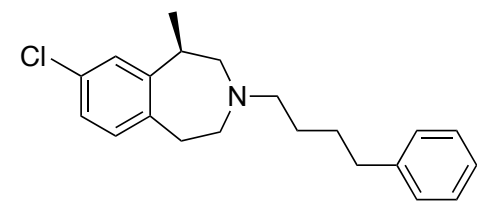

(22): A $20 \mathrm{~mL}$ vial was charged with Lorcaserin hydrochloride (302 mg, $1.30 \mathrm{mmol}), \mathrm{K}_{2} \mathrm{CO}_{3}(413 \mathrm{mg}, 2.99 \mathrm{mmol}), \mathrm{KI}$ (22 mg, $\left.0.13 \mathrm{mmol}\right), \mathrm{MeCN}$ (1.5 mL), and a stir bar. To this mixture, 1-bromo-4-phenylbutane $(277 \mathrm{mg}, 1.30 \mathrm{mmol})$ was added as a solution in $\mathrm{MeCN}(1.5 \mathrm{~mL})$. The vial was sealed with a Teflon cap and heated at $90{ }^{\circ} \mathrm{C}$ for $18 \mathrm{~h}$. The reaction mixture was cooled to room temperature, filtered, and concentrated in vacuo. Purification by flash chromatography (elution gradient from hexanes to $5 \% \mathrm{NEt}_{3} / 10 \%$ EtOAc in hexanes) gave the title compound as a pale yellow oil (298 mg, 70\% yield). ${ }^{1} \mathbf{H} \mathbf{N M R}\left(500 \mathrm{MHz}, \mathrm{CDCl}_{3}\right) \delta 7.30$ $7.26(\mathrm{~m}, 2 \mathrm{H}), 7.21-7.16$ (multiple peaks, 3H), $7.13(\mathrm{~d}, J=2.0 \mathrm{~Hz}, 1 \mathrm{H}), 7.08(\mathrm{dd}, J=7.9,2.1 \mathrm{~Hz}$, 1H), $7.00(\mathrm{~d}, J=8.0 \mathrm{~Hz}, 1 \mathrm{H}), 3.11(\mathrm{p}, J=7.3 \mathrm{~Hz}, 1 \mathrm{H}), 3.02-2.95(\mathrm{~m}, 1 \mathrm{H}), 2.86-2.78$ (multiple peaks, 2H), $2.68(\mathrm{~d}, J=12.2 \mathrm{~Hz}, 1 \mathrm{H}), 2.63(\mathrm{t}, J=7.6 \mathrm{~Hz}, 2 \mathrm{H}), 2.50-2.42(\mathrm{~m}, 2 \mathrm{H}), 2.36-2.24$ (multiple peaks, 2H), 1.68-1.60 (m, 2H), 1.57-1.49 (m, 2H), $1.33(\mathrm{~d}, J=7.3 \mathrm{~Hz}, 3 \mathrm{H}) .{ }^{13} \mathbf{C}$ NMR $\left(125 \mathrm{MHz}, \mathrm{CDCl}_{3}\right) \delta 147.8,142.7,140.2,132.0,130.6,128.5,128.4,126.0,125.9,125.8,62.3$, 59.2, 55.2, 38.5, 36.0, 36.0, 29.5, 26.7, 18.6. HRMS (ESI) $\mathrm{m} / \mathrm{z}$ calc for $\left[\mathrm{C}_{21} \mathrm{H}_{26} \mathrm{CINH}\right]^{+} 328.1827$, found 328.1829 . 


\section{General Procedure III for $N$-Alkylation of Amines}

A $50 \mathrm{~mL}$ round bottom flask was charged with a stir bar followed by a solution of phenylbutylbromide $(2.2 \mathrm{mmol})$ and amine $(11.0 \mathrm{mmol})$ in ethanol $(10 \mathrm{~mL})$, and was heated at reflux for $24 \mathrm{~h}$. The reaction mixture was allowed to cool to room temperature and concentrated. The resulting crude material was dissolved in $0.1 \mathrm{M} \mathrm{NaOH}_{(\mathrm{aq})}(5 \mathrm{~mL})$ and extracted with EtOAc (3 x $5 \mathrm{~mL}$ ). The organic layer was dried over $\mathrm{MgSO}_{4}$ filtered and concentrated. The crude residue was purified by Biotage using a $25 \mathrm{~g}$ Ultra SNAP normal phase cartridge.

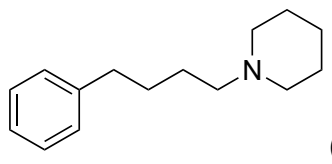

(16): Eluted with $50 \%$ of $\mathrm{Et}_{3} \mathrm{~N} / \mathrm{EtOAc} /$ hexanes $(1: 10: 10)$ in hexanes to give a clear oil (373 mg, 73\% yield). ${ }^{1} \mathbf{H}$ NMR (500 MHz, $\left.\mathrm{CDCl}_{3}\right) \delta$ 7.30-7.21 (m, 2H), 7.20-7.10 (multiple peaks, 3H), 2.62 (t, $J=7.5 \mathrm{~Hz}, 2 \mathrm{H}), 2.34$ (br. s, 4H), 2.32-2.20 (m, 2H), 1.58 (1.72-1.48 (multiple peaks, 8H), 1.47-1.36 (m, 2H). ${ }^{13} \mathbf{C}$ NMR $\left(125 \mathrm{MHz}, \mathrm{CDCl}_{3}\right) \delta 143.0,128.8,128.6$, $126.0,59.9,55.1,36.3,30.0,27.1,26.5,24.9$. This compound has been previously reported. ${ }^{5}$

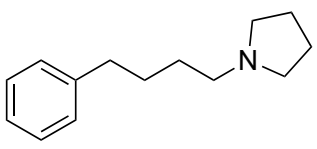

(17): Eluted with $50 \%$ of $\mathrm{Et}_{3} \mathrm{~N} / \mathrm{EtOAc} /$ hexanes $(1: 10: 10)$ in hexanes to give a yellow oil (333 mg, 74\% yield). ${ }^{1} \mathbf{H}$ NMR (500 MHz, $\left.\mathrm{CDCl}_{3}\right) \delta$ 7.31-7.22 (m, 2H), 7.20-7.14 (multiple peaks, 3H), $2.63(\mathrm{t}, J=7.6 \mathrm{~Hz}, 2 \mathrm{H}), 2.54-2.39$ (multiple peaks, $6 \mathrm{H}), 1.83-1.71(\mathrm{~m}, 4 \mathrm{H})$, $1.65(\mathrm{p}, J=7.6 \mathrm{~Hz}, 2 \mathrm{H}), 1.61-1.52(\mathrm{~m}, 2 \mathrm{H}) .{ }^{13} \mathbf{C} \mathbf{N M R}\left(125 \mathrm{MHz}, \mathrm{CDCl}_{3}\right) \delta 142.7,128.5,128.4$, $125.8,56.7,54.4,36.0,29.7,29.0,23.6$. HRMS (ESI) $\mathrm{m} / z$ calc for $\left[\mathrm{C}_{14} \mathrm{H}_{21} \mathrm{NH}\right]^{+} 204.1747$, found 204.1743

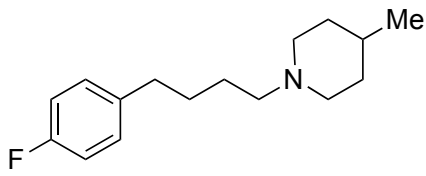

(25): Eluted with $50 \%$ of $\mathrm{Et}_{3} \mathrm{~N} / \mathrm{EtOAc} /$ hexanes $(1: 10: 10)$ in hexanes to furnish a clear oil (381 mg, 69\% yield). ${ }^{1} \mathbf{H}$ NMR (400 MHz, $\left.\mathrm{CDCl}_{3}\right) \delta$ 7.14-7.09 (m, 2H), 6.97$6.91(\mathrm{~m}, 2 \mathrm{H}), 2.90-2.80(\mathrm{~m}, 2 \mathrm{H}), 2.59$ (t, $J=7.4 \mathrm{~Hz}, 2 \mathrm{H}), 2.33-2.27$ (m, 2H), 1.92-1.80 (m, 2H), 1.63-1.47 (multiple peaks, 6H), 1.41-1.26 (m, 1H), 1.30-1.15 (m, 2H), $0.91(\mathrm{~d}, J=6.3 \mathrm{~Hz}, 3 \mathrm{H}) .{ }^{13} \mathbf{C}$ NMR $\left(100 \mathrm{MHz}, \mathrm{CDCl}_{3}\right) \delta 161.3\left(\mathrm{~d}, J_{\mathrm{C}-\mathrm{F}}=243.0 \mathrm{~Hz}\right), 138.3\left(\mathrm{~d}, J_{\mathrm{C}-\mathrm{F}}=3.1 \mathrm{~Hz}\right), 129.8\left(\mathrm{~d}, J_{\mathrm{C}-\mathrm{F}}=7.8\right.$ $\mathrm{Hz}), 115.1\left(\mathrm{~d}, J_{\mathrm{C}-\mathrm{F}}=21.1 \mathrm{~Hz}\right), 59.2,54.3,35.2,34.5,31.0,29.9,26.9,22.1 .{ }^{19} \mathbf{F}$ NMR $(376 \mathrm{MHz}$, $\left.\mathrm{CDCl}_{3}\right) \delta-118.2$. HRMS (ESI) $\mathrm{m} / z$ calc for $\left[\mathrm{C}_{16} \mathrm{H}_{24} \mathrm{FNH}\right]^{+} 250.1966$, found 250.1963 . 


\section{General Procedure for Amine Oxyfunctionalization}

To a 1 dram glass vial charged with a stir bar and amine $(0.25 \mathrm{mmol}), \mathrm{CF}_{3} \mathrm{CO}_{2} \mathrm{H}(21 \mu \mathrm{L}$, $0.275 \mathrm{mmol}$ ) was added, and the vial was tightly capped with a Teflon lined cap and left to stir for about 5 min. Next, pyridine/MeCN (1:5) $(0.40 \mathrm{~mL})$ was added, and the resulting mixture was thoroughly mixed until it was homogeneous. In certain cases $\mathrm{H}_{2} \mathrm{O}(75 \mu \mathrm{L})$ was added to aid dissolution. A solution of $\mathrm{FeCl}_{3} \cdot 6 \mathrm{H}_{2} \mathrm{O}(3.4 \mathrm{mg}, 0.0125 \mathrm{mmol})$ and 2-picolinic acid $(3.8 \mathrm{mg}, 0.313$ mol) in pyridine/MeCN (1:5) $(0.10 \mathrm{~mL})$ was added. The cap was replaced with a Teflon-lined septum-containing cap. The cap was loosely fitted to avoid pressure build-up during the course of the reaction. ${ }^{t} \mathrm{BuOOH}\left(70 \mathrm{wt} \%\right.$ in $\left.\mathrm{H}_{2} \mathrm{O}, 0.62 \mathrm{~mL}, 4.50 \mathrm{mmol}\right)$ was added by syringe pump over 15 $\mathrm{h}$. After a total reaction time of $48 \mathrm{~h}$, the presence of residual peroxide was tested with peroxide test strips. In most cases the reaction mixture tested negative and if the peroxide test was positive, the reaction mixture was quenched with a saturated aqueous solution of $\mathrm{Na}_{2} \mathrm{~S}_{2} \mathrm{O}_{3}$ until the peroxide test was negative. The reaction mixture was concentrated on the rotary evaporator, and the resulting crude material was purified by Biotage using a $10 \mathrm{~g}$ Ultra SNAP normal phase cartridge, eluting with the $\mathrm{Et}_{3} \mathrm{~N} / \mathrm{EtOAc} /$ hexanes solvent mixture indicated. All isolated yields are the average of two runs.

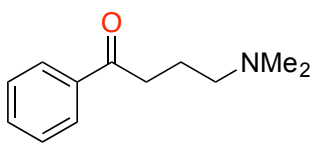

(1a): Eluted with $\mathrm{Et}_{3} \mathrm{~N} / \mathrm{EtOAc} / \mathrm{h}$ exanes $(1: 10: 10)$ to give a clear oil $(28 \mathrm{mg}$, $60 \%$ yield). ${ }^{1} \mathbf{H}$ NMR $\left(400 \mathrm{MHz}, \mathrm{CDCl}_{3}\right) \delta 8.02-7.91(\mathrm{~m}, 2 \mathrm{H}), 7.53(\mathrm{t}, J=7.4 \mathrm{~Hz}, 1 \mathrm{H}), 7.48-7.35$ (m, 2H), 3.01 (t, $J=7.3 \mathrm{~Hz}, 2 \mathrm{H}), 2.34$ (t, $J=7.1 \mathrm{~Hz}, 2 \mathrm{H}), 2.21(\mathrm{~s}, 6 \mathrm{H}), 1.90$ (p, $J=7.2 \mathrm{~Hz}, 2 \mathrm{H})$. ${ }^{13}$ C NMR $\left(100 \mathrm{MHz}, \mathrm{CDCl}_{3}\right) \delta 200.2,137.2,133.0,128.6,128.2,59.1,45.5,36.4,22.3 . \mathbf{I R}\left(\mathrm{cm}^{-1}\right)$ 3059, 2941, 1683, 1597, 1448, 1222. HRMS (ESI) $m / z$ calc for $\left[\mathrm{C}_{12} \mathrm{H}_{17} \mathrm{NOH}\right]^{+} 192.1383$, found 192.1378 .

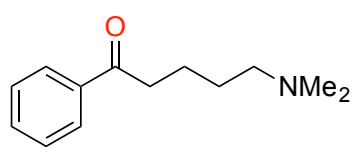

(2a): Eluted with $\mathrm{Et}_{3} \mathrm{~N} / \mathrm{EtOAc} /$ hexanes $(1: 10: 10)$ to give a clear oil $(29 \mathrm{mg}$, $56 \%$ yield). ${ }^{1} \mathbf{H}$ NMR $\left(400 \mathrm{MHz}, \mathrm{CDCl}_{3}\right) \delta 7.98-7.94(\mathrm{~m}, 2 \mathrm{H}), 7.55(\mathrm{t}, J=7.4 \mathrm{~Hz}, 1 \mathrm{H}), 7.45(\mathrm{t}, J$ $=7.5 \mathrm{~Hz}, 2 \mathrm{H}), 3.00(\mathrm{t}, J=7.3 \mathrm{~Hz}, 2 \mathrm{H}), 2.33-2.27(\mathrm{~m}, 2 \mathrm{H}), 2.21(\mathrm{~s}, 6 \mathrm{H}), 1.76(\mathrm{p}, J=7.3 \mathrm{~Hz}, 2 \mathrm{H})$, $1.60-1.51(\mathrm{~m}, 2 \mathrm{H}) .{ }^{13} \mathbf{C}$ NMR $\left(100 \mathrm{MHz}, \mathrm{CDCl}_{3}\right) \delta 200.3,137.1,133.0,128.7,128.1,59.7,45.6$, 38.5, 27.5, 22.3. IR $\left(\mathrm{cm}^{-1}\right) 3059,2939,1682,1597,1447,1215$. HRMS (ESI) $\mathrm{m} / z$ calc for $\left[\mathrm{C}_{13} \mathrm{H}_{19} \mathrm{NOH}\right]^{+}$206.1539, found 206.1535. 


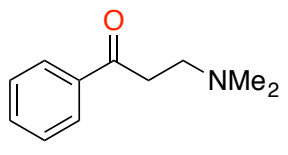

(3a): Crude ${ }^{1} \mathrm{H}$ NMR spectroscopic analysis showed $38 \%$ yield of $\mathbf{3 a}$ and $14 \%$

of phenyl vinyl ketone. The crude material was eluted with $\mathrm{Et}_{3} \mathrm{~N} / \mathrm{EtOAc} / \mathrm{hexanes}(1: 10: 10)$ to give 3a as a yellowish oil (9 mg, 20\% yield). ${ }^{1} \mathbf{H}$ NMR (400 MHz, $\left.\mathrm{CDCl}_{3}\right) \delta 7.97(\mathrm{~d}, J=7.7 \mathrm{~Hz}, 2 \mathrm{H})$, 7.60-7.52 (m, 1H), 7.46 (t, $J=7.6 \mathrm{~Hz}, 2 \mathrm{H}), 3.16$ (t, $J=7.4 \mathrm{~Hz}, 2 \mathrm{H}), 2.76$ (t, $J=7.4 \mathrm{~Hz}, 2 \mathrm{H}), 2.29$ (s, 6H). ${ }^{13} \mathbf{C}$ NMR $\left(100 \mathrm{MHz}, \mathrm{CDCl}_{3}\right) \delta 199.3,137.1,133.2,128.8,128.2,54.5,45.7,37.1$. IR $\left(\mathrm{cm}^{-1}\right) 3057,2941,1679,1596,1448,1378,1206$. This compound has been previously reported. ${ }^{6}$

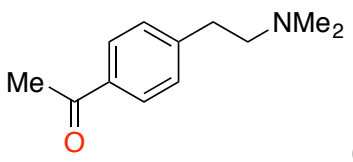

(6a): Reaction was run using a different amount of ${ }^{t} \mathrm{BuOOH}(0.41 \mathrm{~mL}, 3.0$ mmol, 12 equiv) with slow addition over $10 \mathrm{~h}$. The crude material was eluted with $\mathrm{Et}_{3} \mathrm{~N} / \mathrm{EtOAc} / \mathrm{hexanes}(1: 10: 10)$ to give a clear oil $\left(30 \mathrm{mg}, 63 \%\right.$ yield). ${ }^{1} \mathbf{H}$ NMR (400 MHz, $\left.\mathrm{CDCl}_{3}\right) \delta 7.86(\mathrm{~d}, J=8.2 \mathrm{~Hz}, 2 \mathrm{H}), 7.28(\mathrm{~d}, J=8.2 \mathrm{~Hz}, 2 \mathrm{H}), 2.85-2.78(\mathrm{~m}, 2 \mathrm{H}), 2.56(\mathrm{~s}, 3 \mathrm{H}), 2.54-$ 2.49 (m, 2H), 2.27 (s, 6H). ${ }^{13} \mathbf{C}$ NMR $\left(100 \mathrm{MHz}, \mathrm{CDCl}_{3}\right) \delta 197.9,146.4,135.3,128.9,128.6,61.0$, 45.5, 34.4, 26.6. IR $\left(\mathrm{cm}^{-1}\right)$ 2944, 1679, 1606, 1460, 1356, 1265. HRMS (ESI) $\mathrm{m} / z$ calc for $\left[\mathrm{C}_{12} \mathrm{H}_{17} \mathrm{NOH}\right]^{+}$192.1383, found 192.1378 .

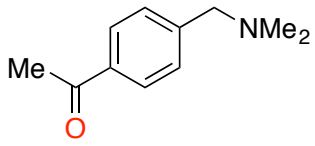

(7a): Reaction was run using a different amount of ${ }^{t} \mathrm{BuOOH}(0.41 \mathrm{~mL}, 3.0$ mmol, 12 equiv) with slow addition over $10 \mathrm{~h}$. The crude material was eluted with $\mathrm{Et}_{3} \mathrm{~N} / \mathrm{EtOAc} / \mathrm{hexanes}(1: 10: 10)$ to give a clear oil $\left(26 \mathrm{mg}, 60 \%\right.$ yield). ${ }^{1} \mathbf{H}$ NMR (400 MHz, $\left.\mathrm{CDCl}_{3}\right) \delta 7.90(\mathrm{~d}, J=8.3 \mathrm{~Hz}, 2 \mathrm{H}), 7.39(\mathrm{~d}, J=8.3 \mathrm{~Hz}, 2 \mathrm{H}), 3.45(\mathrm{~s}, 2 \mathrm{H}), 2.58(\mathrm{~s}, 3 \mathrm{H}), 2.23(\mathrm{~s}, 6 \mathrm{H})$. ${ }^{13}$ C NMR $\left(100 \mathrm{MHz}, \mathrm{CDCl}_{3}\right) \delta 198.0,144.8,136.2,129.2,128.5,64.1,45.6,26.7$. IR $\left(\mathrm{cm}^{-1}\right) 2942$, $1680,1606,1357,1265$. HRMS (ESI) $m / z$ calc for $\left[\mathrm{C}_{11} \mathrm{H}_{15} \mathrm{NOH}\right]^{+} 178.1226$, found 178.1221 .

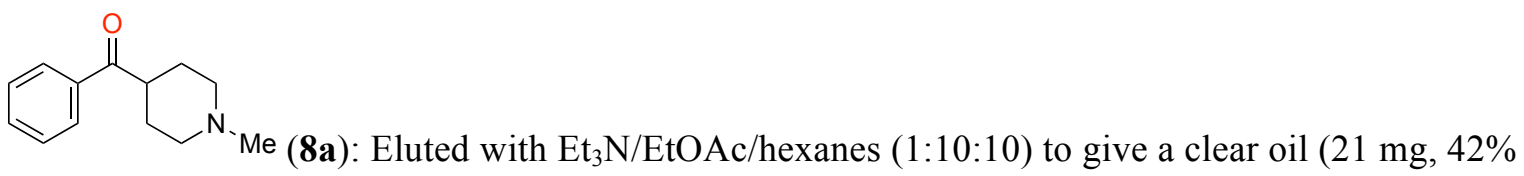
yield). ${ }^{1}$ H NMR (400 MHz, $\left.\mathrm{CDCl}_{3}\right) \delta$ 7.94-7.89 (m, 2H), $7.54(\mathrm{t}, J=7.4 \mathrm{~Hz}, 1 \mathrm{H}), 7.45$ (t, $J=7.5$ Hz, 2H), 3.26-3.14 (m, 1H), 2.92 (br. dt, $J=11.2,2.6 \mathrm{~Hz}, 2 \mathrm{H}), 2.29$ (s, 3H), 2.11-2.02 (m, 2H), 1.89-1.80 (multiple peaks, 4H). ${ }^{13} \mathbf{C}$ NMR $\left(100 \mathrm{MHz}, \mathrm{CDCl}_{3}\right) \delta 202.8,136.2,133.0,128.7,128.3$, 
55.4, 46.6, 43.3, 28.9. IR $\left(\mathrm{cm}^{-1}\right)$ 3059, 2935, 1677, 1580, 1447, 1374, 1264. HRMS (ESI) $\mathrm{m} / z$ calc for $\left[\mathrm{C}_{13} \mathrm{H}_{17} \mathrm{NOH}\right]^{+} 204.1383$, found 204.1378 .

(1:10:10) to give a white solid (30 mg, 51\% yield). ${ }^{1} \mathbf{H}$ NMR $\left(400 \mathrm{MHz}, \mathrm{CDCl}_{3}\right) \delta 7.93(\mathrm{~d}, J=8.9 \mathrm{~Hz}, 2 \mathrm{H}), 6.94(\mathrm{~d}, J=8.9 \mathrm{~Hz}, 2 \mathrm{H})$, 3.87 (s, 3H), 3.17 (p, $J=7.7 \mathrm{~Hz}, 1 \mathrm{H}), 2.94$ (br. dt, $J=11.0,2.6 \mathrm{~Hz}, 2 \mathrm{H}), 2.31$ (s, 3H), 2.21-1.99 (m, 2H), 1.94-1.80 (m, 4H). ${ }^{13} \mathbf{C}$ NMR (100 MHz, $\left.\mathrm{CDCl}_{3}\right) \delta$ 201.3, 163.4, 130.6, 129.1, 113.9, 55.6, 55.5, 46.6, 42.9, 29.0. IR $\left(\mathrm{cm}^{-1}\right)$ 3005, 2935, 1660, 1599, 1375, 1256, 1178. HRMS (ESI) $m / z$ calc for $\left[\mathrm{C}_{14} \mathrm{H}_{19} \mathrm{NO}_{2} \mathrm{H}\right]^{+} 234.1489$, found 234.1483 .<smiles>N#CC1CCC(C(=O)c2ccc(F)cc2)CC1</smiles>
(10a): Eluted with $\mathrm{Et}_{3} \mathrm{~N} / \mathrm{EtOAc} /$ hexanes $(1: 10: 10)$ to give a mixture of product and starting material that was further eluted with $50 \% \mathrm{Et}_{3} \mathrm{~N} / \mathrm{EtOAc} /$ hexanes $(1: 10: 10)$ in hexanes to give a white solid $\left(21 \mathrm{mg}, 37 \%\right.$ yield). ${ }^{1} \mathbf{H}$ NMR $\left(400 \mathrm{MHz}, \mathrm{CDCl}_{3}\right) \delta$ 8.01-7.93 (m, 2H), 7.15 (t, $J=8.6 \mathrm{~Hz}, 2 \mathrm{H}), 3.18$ (p, $J=7.4 \mathrm{~Hz}, 1 \mathrm{H}), 2.95$ (br. dt, $J=11.2,2.7 \mathrm{~Hz}, 2 \mathrm{H}), 2.33$ (s, $3 \mathrm{H}), 2.14-2.03(\mathrm{~m}, 2 \mathrm{H}), 1.94-1.80(\mathrm{~m}, 4 \mathrm{H}) .{ }^{13} \mathbf{C}$ NMR $\left(100 \mathrm{MHz}, \mathrm{CDCl}_{3}\right) \delta 201.2,165.7\left(\mathrm{~d}, J_{\mathrm{C}-\mathrm{F}}=\right.$ $254.5 \mathrm{~Hz}), 132.6\left(\mathrm{~d}, J_{\mathrm{C}-\mathrm{F}}=3.0 \mathrm{~Hz}\right), 131.0\left(\mathrm{~d}, J_{\mathrm{C}-\mathrm{F}}=9.2 \mathrm{~Hz}\right), 115.9\left(\mathrm{~d}, J_{\mathrm{C}-\mathrm{F}}=21.8 \mathrm{~Hz}\right), 55.4,46.6$, 43.3, 28.9. ${ }^{19}$ F NMR $\left(377 \mathrm{MHz}, \mathrm{CDCl}_{3}\right) \delta-105.6 . \mathbf{I R}\left(\mathrm{cm}^{-1}\right)$ 3032, 2940, 1665, 1595, 1446, 1375 , 1228, 1138. HRMS (ESI) $m / z$ calc for $\left[\mathrm{C}_{13} \mathrm{H}_{16} \mathrm{FNOH}\right]^{+} 222.1289$, found 222.1285 .

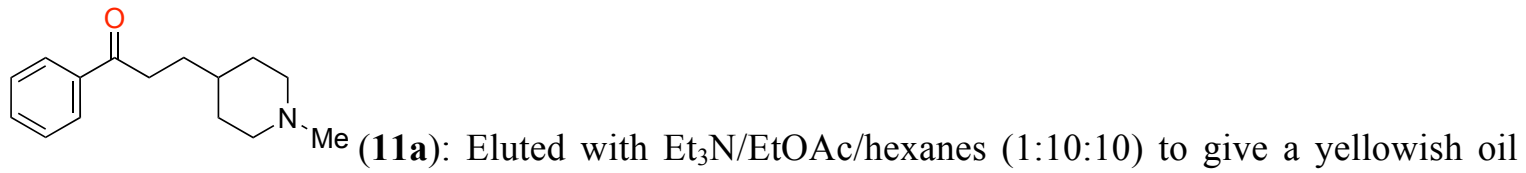
(37 mg, 64\% yield). ${ }^{1} \mathbf{H}$ NMR (400 MHz, $\left.\mathrm{CDCl}_{3}\right) \delta$ 7.98-7.89 (m, 2H), $7.55(\mathrm{t}, J=7.4 \mathrm{~Hz}, 1 \mathrm{H})$, 7.45 (t, $J=7.5 \mathrm{~Hz}, 2 \mathrm{H}), 3.01-2.95$ (m, 2H), 2.84 (br. d, $J=11.4 \mathrm{~Hz}, 2 \mathrm{H}), 2.25$ (s, 3H), 1.90 (br. t, $J$ $=11.3 \mathrm{~Hz}, 2 \mathrm{H}$ ), 1.76-1.64 (multiple peaks, 4H), 1.37-1.26 (multiple peaks, 3H). ${ }^{13} \mathbf{C}$ NMR (100 $\left.\mathrm{MHz}, \mathrm{CDCl}_{3}\right) \delta 200.6,137.1,133.1,128.7,128.2,56.0,46.6,36.0,34.9,32.4,30.9$. IR $\left(\mathrm{cm}^{-1}\right)$ 3064, 2928, 1682, 1597, 1448, 1378, 1278. HRMS (ESI) $\mathrm{m} / z$ calc for $\left[\mathrm{C}_{15} \mathrm{H}_{21} \mathrm{NOH}\right]^{+} 232.1696$, found 232.1690 . 
(12a): Eluted with $\mathrm{Et}_{3} \mathrm{~N} /$ EtOAc/hexanes (1:10:10) to give a clear oil (34 mg, 44\% yield). ${ }^{1} \mathbf{H}$ NMR (400 MHz, $\left.\mathrm{CDCl}_{3}\right) \delta$ ) 7.97-7.93 (m, 2H), $7.55(\mathrm{t}, J=7.4 \mathrm{~Hz}, 1 \mathrm{H})$, 7.49-7.42 (m 2H), 7.31 (app. d, $J=4.4 \mathrm{~Hz}, 4 \mathrm{H}), 7.29-7.20$ (m, 1H), 3.49 (s, 2H), 3.05-2.94 (m, 2H), 2.88 (br. d, $J=10.9 \mathrm{~Hz}, 2 \mathrm{H}$ ), 1.94 (br. t, $J=10.9 \mathrm{~Hz}, 2 \mathrm{H}$ ), 1.74-1.62 (multiple peaks, 4H), 1.41-1.21 (multiple peaks, 3H). ${ }^{13} \mathbf{C}$ NMR $\left(100 \mathrm{MHz}, \mathrm{CDCl}_{3}\right) \delta 200.6,138.7,137.1,133.0,129.3$, 128.7, 128.3, 128.2, 127.0, 63.6, 54.0, 36.1, 35.6, 32.4, 31.0. IR $\left(\mathrm{cm}^{-1}\right)$ 3059, 2920, 1682, 1597, $1448,1366,1265$. HRMS (ESI) $\mathrm{m} / z$ calc for $\left[\mathrm{C}_{21} \mathrm{H}_{25} \mathrm{NOH}\right]^{+} 308.2009$, found 308.2005.

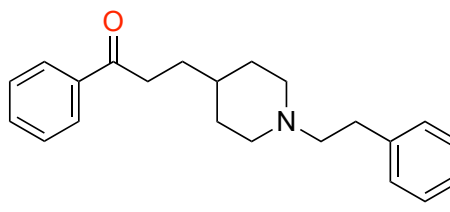

(13a): Eluted with $\mathrm{Et}_{3} \mathrm{~N} / \mathrm{EtOAc} /$ hexanes $(1: 10: 10)$ to give a clear oil (37 mg, 47\% yield). ${ }^{1} \mathbf{H}$ NMR (400 MHz, $\left.\mathrm{CDCl}_{3}\right) \delta 8.03-7.91(\mathrm{~m}, 2 \mathrm{H}), 7.56(\mathrm{t}, J=7.4 \mathrm{~Hz}, 1 \mathrm{H})$, 7.51-7.43 (m, 2H), 7.33-7.23 (m, 2H), 7.23-7.15 (multiple peaks, 3H), 3.09-2.95 (m, 4H), 2.87$2.77(\mathrm{~m}, 2 \mathrm{H}), 2.66-2.54(\mathrm{~m}, 2 \mathrm{H}), 2.09-1.93(\mathrm{~m}, 2 \mathrm{H}), 1.85-1.67$ (multiple peaks, 4H), 1.46-1.30 (multiple peaks, 3H). ${ }^{13} \mathrm{C}$ NMR $\left(100 \mathrm{MHz}, \mathrm{CDCl}_{3}\right) \delta 200.5,140.7,137.1,133.0,128.8,128.7$, 128.5, 128.1, 126.1, 61.1, 54.0, 35.9, 35.5, 34.0, 32.4, 30.9. IR $\left(\mathrm{cm}^{-1}\right) 3027,2921,1682,1596$, 1448, 1368. 1272. HRMS (ESI) $\mathrm{m} / z$ calc for $\left[\mathrm{C}_{22} \mathrm{H}_{27} \mathrm{NOH}\right]^{+} 322.2165$, found 322.2161 .

(14a): Eluted with $\mathrm{Et}_{3} \mathrm{~N} /$ EtOAc/hexanes $(1: 10: 10)$ to give a clear oil (35mg, 54\% yield). ${ }^{1} \mathbf{H}$ NMR $\left(400 \mathrm{MHz}, \mathrm{CDCl}_{3}\right) \delta$ 7.99-7.92 (m, 2H), 7.60-7.51 (m, 1H), $7.46(\mathrm{t}, J$ $=7.5 \mathrm{~Hz}, 2 \mathrm{H}), 5.89$ (ddt, $J=16.9,10.2,6.6 \mathrm{~Hz}, 1 \mathrm{H}), 5.22-5.09(\mathrm{~m}, 2 \mathrm{H}), 3.03-2.89$ (multiple peaks, $6 \mathrm{H}$ ), 1.90 (br. t, $J=11.0 \mathrm{~Hz}, 2 \mathrm{H}$ ), 1.79-1.65 (multiple peaks, 4H), 1.38-1.28 (multiple peaks, 3H). ${ }^{13}$ C NMR $\left(100 \mathrm{MHz}, \mathrm{CDCl}_{3}\right) \delta 200.1,137.1,135.7,133.1,128.7,128.2,117.7,62.3,53.9,36.0$, 35.4, 32.3, 31.0. IR ( $\left.\mathrm{cm}^{-1}\right) 3059$, 2920, 1682, 1644, 1596, 1447, 1331, 1270. HRMS (ESI) $\mathrm{m} / z$ calc for $\left[\mathrm{C}_{17} \mathrm{H}_{23} \mathrm{NOH}\right]^{+} 258.1852$, found 258.1847 .<smiles>N#CCCCN1CCC(CCC(=O)c2ccccc2)CC1</smiles>

(15a): Eluted with $\mathrm{Et}_{3} \mathrm{~N} / \mathrm{EtOAc} /$ hexanes $(1: 10: 10)$ to give a clear oil (34 mg, 47\% yield). ${ }^{1} \mathbf{H}$ NMR (400 MHz, $\left.\mathrm{CDCl}_{3}\right) \delta$ 7.98-7.92 (m, 2H), 7.60-7.50 (m, 1H), 7.45 (t, 
$J=7.5 \mathrm{~Hz}, 2 \mathrm{H}), 3.02-2.95$ (m, 2H), 2.85 (br. d, $J=11.7 \mathrm{~Hz}, 2 \mathrm{H}), 2.40$ (td, $J=7.0,2.9 \mathrm{~Hz}, 4 \mathrm{H})$, 1.97-1.88 (m, 2H), 1.81 (p, $J=7.0 \mathrm{~Hz}, 2 \mathrm{H}), 1.75-1.62$ (multiple peaks, 4H), 1.39-1.19 (multiple peaks, 3H). ${ }^{13} \mathbf{C}$ NMR $\left(100 \mathrm{MHz}, \mathrm{CDCl}_{3}\right) \delta 200.5,137.1,133.1,128.7,128.1,120.0,56.8,53.9$, 36.0, 35.5, 32.3, 30.9, 23.1, 15.1. IR $\left(\mathrm{cm}^{-1}\right)$ 3058, 2928, 1681, 1596, 1447, 1347, 1272. HRMS (ESI) $m / z$ calc for $\left[\mathrm{C}_{18} \mathrm{H}_{24} \mathrm{~N}_{2} \mathrm{OH}\right]^{+} 285.1961$, found 285.1956 .

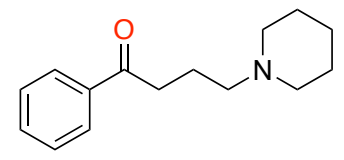

(16a): Eluted with $\mathrm{Et}_{3} \mathrm{~N} / \mathrm{EtOAc} /$ hexanes (1:10:10) to give a yellowish oil (36 mg, 62\% yield). ${ }^{1} \mathbf{H}$ NMR $\left(500 \mathrm{MHz}, \mathrm{CDCl}_{3}\right) \delta$ 7.99-7.96 (m, 2H), $7.55(\mathrm{t}, J=7.4 \mathrm{~Hz}, 1 \mathrm{H}), 7.48-$ $7.43(\mathrm{~m}, 2 \mathrm{H}), 2.99$ (t, $J=7.3 \mathrm{~Hz}, 2 \mathrm{H}), 2.48-2.27$ (multiple peaks, $6 \mathrm{H}), 1.94$ (p, $J=7.3 \mathrm{~Hz}, 2 \mathrm{H}$ ), $1.54(\mathrm{p}, J=5.6 \mathrm{~Hz}, 4 \mathrm{H}), 1.44-1.37(\mathrm{~m}, 2 \mathrm{H}) .{ }^{13} \mathbf{C} \mathbf{N M R}\left(125 \mathrm{MHz}, \mathrm{CDCl}_{3}\right) \delta 200.4,137.3,133.0$, 128.7, 128.2, 58.7, 54.7, 36.7, 26.1, 24.6, 21.9. IR ( $\left.\mathrm{cm}^{-1}\right)$ 3059, 2929, 1683, 1597, 1448, 1350, 1225, 1122. HRMS (ESI) $\mathrm{m} / z$ calc for $\left[\mathrm{C}_{15} \mathrm{H}_{21} \mathrm{NOH}\right]^{+} 232.1696$, found 232.1694 .

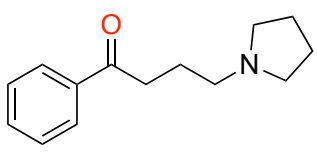

(17a): Eluted with $\mathrm{Et}_{3} \mathrm{~N} / \mathrm{EtOAc} /$ hexanes (1:10:10) to give a yellowish oil (38 $\mathrm{mg}, 68 \%$ yield). ${ }^{1} \mathbf{H}$ NMR $\left(400 \mathrm{MHz}, \mathrm{CDCl}_{3}\right) \delta 8.02-7.92(\mathrm{~m}, 2 \mathrm{H}), 7.58-7.51(\mathrm{~m}, 1 \mathrm{H}), 7.45(\mathrm{t}, J=$ $7.5 \mathrm{~Hz}, 2 \mathrm{H}$ ), 3.03 (t, $J=7.3 \mathrm{~Hz}, 2 \mathrm{H}$ ), 2.58-2.43 (multiple peaks, 6H), 1.96 (p, $J=7.3 \mathrm{~Hz}, 2 \mathrm{H}$ ), 1.83-1.68 (multiple peaks, 4H). ${ }^{13} \mathbf{C}$ NMR $\left(125 \mathrm{MHz} \mathrm{CDCl}_{3}\right) \delta 200.0,136.9,132.7,128.3,127.9$, 55.6, 53.9, 36.4, 23.5, 23.3. IR $\left(\mathrm{cm}^{-1}\right)$ 3059, 2960, 1683, 1597, 1448, 1350, 1211, 1145. HRMS (ESI) $m / z$ calc for $\left[\mathrm{C}_{14} \mathrm{H}_{19} \mathrm{NOH}\right]^{+} 218.1539$, found 218.1540 .

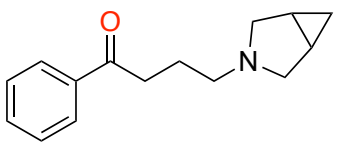

(18a): Elution with $2 \% \mathrm{NEt}_{3} / 10 \%$ EtOAc in hexanes gave the title product as a colorless oil (26 mg, 45\% yield). ${ }^{1} \mathbf{H} \mathbf{N M R}\left(500 \mathrm{MHz}, \mathrm{CDCl}_{3}\right) \delta 7.96(\mathrm{~d}, J=7.5 \mathrm{~Hz}, 2 \mathrm{H}), 7.54$ (t, $J=7.3 \mathrm{~Hz}, 1 \mathrm{H}), 7.45$ (t, $J=7.7 \mathrm{~Hz}, 2 \mathrm{H}$ ), 3.00-2.94 (multiple peaks, 4H), 2.47 (t, $J=7.0 \mathrm{~Hz}$, 2H), 2.29-2.23 (m, 2H), 1.87 (p, $J=7.1 \mathrm{~Hz}, 2 \mathrm{H}), 1.33-1.27$ (m, 2H), 0.59 (q, J=3.8 Hz, 1H), 0.29 $(\mathrm{td}, J=7.7,4.1 \mathrm{~Hz}, 1 \mathrm{H}) .{ }^{13} \mathrm{C}$ NMR $\left(125 \mathrm{MHz}, \mathrm{CDCl}_{3}\right) \delta 200.5,137.4,132.9,128.6,128.2,55.1$, 54.7, 36.3, 23.8, 15.4, 7.0. IR $\left(\mathrm{cm}^{-1}\right)$ 3063, 2784, 1682, 1597, 1447, 1358, 1215, 1170. HRMS (ESI) $m / z$ calc'd for $\left[\mathrm{C}_{15} \mathrm{H}_{19} \mathrm{NOH}\right]^{+} 230.1539$, found 230.1538 . 


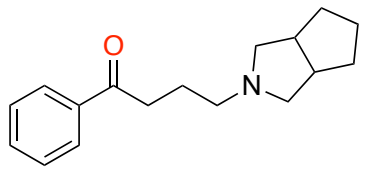

(19a): Elution gradient from $2-4 \% \mathrm{NEt}_{3} / 10 \%$ EtOAc in hexanes gave the title product as a colorless oil (31 mg, 48\% yield). ${ }^{1} \mathbf{H}$ NMR $\left(500 \mathrm{MHz}, \mathrm{CDCl}_{3}\right) \delta 7.97(\mathrm{~d}, J=7.7$ $\mathrm{Hz}, 2 \mathrm{H}), 7.54$ (t, $J=7.3 \mathrm{~Hz}, 1 \mathrm{H}), 7.45(\mathrm{t}, J=7.6 \mathrm{~Hz}, 2 \mathrm{H}), 3.01(\mathrm{t}, J=7.3 \mathrm{~Hz}, 2 \mathrm{H}), 2.72$ (t, $J=7.9$ $\mathrm{Hz}, 2 \mathrm{H}), 2.60-2.50(\mathrm{~m}, 2 \mathrm{H}), 2.42(\mathrm{t}, J=7.2 \mathrm{~Hz}, 2 \mathrm{H}), 2.03(\mathrm{dd}, J=8.9,4.5 \mathrm{~Hz}, 2 \mathrm{H}), 1.92(\mathrm{p}, J=$ $7.2 \mathrm{~Hz}, 2 \mathrm{H}), 1.66-1.30$ (multiple peaks, $6 \mathrm{H}) .{ }^{13} \mathbf{C} \mathbf{N M R}\left(125 \mathrm{MHz}, \mathrm{CDCl}_{3}\right) \delta 200.5,137.3,133.0$, 128.6, 128.2, 61.8, 55.5, 42.5, 36.7, 32.9, 26.1, 23.7. IR $\left(\mathrm{cm}^{-1}\right)$ 2941, 1681, 1597, 1447, 1352, 1216, 1148. HRMS (ESI) $\mathrm{m} / z$ calc for $\left[\mathrm{C}_{17} \mathrm{H}_{23} \mathrm{NOH}\right]^{+} 258.1852$, found: 258.1849 .

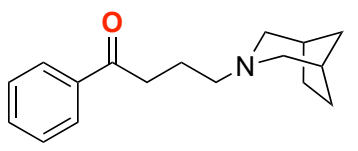

(20a): Eluted with $\mathrm{Et}_{3} \mathrm{~N} / \mathrm{EtOAc} /$ hexanes $(1: 10: 10)$ to give a yellow oil (26 mg, 40\% yield). ${ }^{1} \mathbf{H}$ NMR $\left(500 \mathrm{MHz}, \mathrm{CDCl}_{3}\right) \delta 8.05-7.94(\mathrm{~m}, 2 \mathrm{H}), 7.62-7.52(\mathrm{~m}, 1 \mathrm{H}), 7.46(\mathrm{t}, J=$ $7.7 \mathrm{~Hz}, 2 \mathrm{H}), 3.03$ (t, $J=7.3 \mathrm{~Hz}, 2 \mathrm{H}), 2.69-2.61$ (m, 2H), 2.34 (t, $J=6.8 \mathrm{~Hz}, 2 \mathrm{H}), 2.09$ (br. s, 2H), 1.98 (br. d, $J=10.2 \mathrm{~Hz}, 2 \mathrm{H}), 1.87$ (p, $J=7.0 \mathrm{~Hz}, 2 \mathrm{H}), 1.64-1.49$ (m, 4H), 1.48-1.39 (m, 1H), 1.36$1.30(\mathrm{~m}, 1 \mathrm{H}) .{ }^{13} \mathbf{C}$ NMR $\left(125 \mathrm{MHz}, \mathrm{CDCl}_{3}\right) \delta 200.9,137.4,132.9,128.7,128.2,60.4,57.3,37.9$, 36.2, 35.4, 28.9, 21.9. IR $\left(\mathrm{cm}^{-1}\right) 3039,2932,1680,1596,1448,1362,1210$. HRMS (ESI) $\mathrm{m} / z$ calc for $\left[\mathrm{C}_{17} \mathrm{H}_{23} \mathrm{NOH}\right]^{+}$258.1852, found 258.1853.

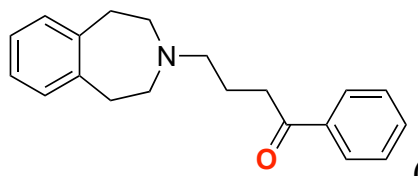

(21a): Eluted with $\mathrm{Et}_{3} \mathrm{~N} / \mathrm{EtOAc} /$ hexanes $(1: 10: 10)$ to give a yellow oil (35 mg, 47\% yield). ${ }^{1} \mathbf{H}$ NMR $\left(400 \mathrm{MHz}, \mathrm{CDCl}_{3}\right) \delta 8.02-7.98(\mathrm{~m}, 2 \mathrm{H}), 7.60-7.51(\mathrm{~m}, 1 \mathrm{H}), 7.46(\mathrm{t}$, $J=7.5 \mathrm{~Hz}, 2 \mathrm{H}), 7.14-7.05$ (multiple peaks, 4H), 3.03 (t, $J=7.1 \mathrm{~Hz}, 2 \mathrm{H}), 2.93-2.83$ (m, 4H), 2.67$2.61(\mathrm{~m}, 4 \mathrm{H}), 2.56(\mathrm{t}, J=7.1 \mathrm{~Hz}, 2 \mathrm{H}), 1.99(\mathrm{p}, J=7.1 \mathrm{~Hz}, 2 \mathrm{H}) .{ }^{13} \mathbf{C} \mathbf{N M R}\left(100 \mathrm{MHz}, \mathrm{CDCl}_{3}\right) \delta$ 200.2, 142.3, 137.3, 132.9, 128.9, 128.6, 128.2, 126.2, 58.2, 55.4, 36.6, 36.4, 22.0. IR ( $\left.\mathrm{cm}^{-1}\right) 3059$, 2932, 1682, 1596, 1493, 1448, 1362, 1210, 1120. HRMS (ESI) $m / z$ calc for $\left[\mathrm{C}_{20} \mathrm{H}_{23} \mathrm{NOH}\right]^{+}$ 294.1852, found 294.1848.

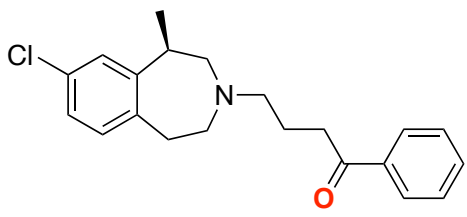

(22a): Elution gradient from $2-5 \% \mathrm{NEt}_{3} / 10 \%$ EtOAc in hexanes gave the title product as a colorless oil $\left(27 \mathrm{mg}, 31 \%\right.$ yield). ${ }^{1} \mathbf{H}$ NMR $\left(500 \mathrm{MHz}, \mathrm{CDCl}_{3}\right) \delta 7.98(\mathrm{~d}$, 
$J=7.2 \mathrm{~Hz}, 2 \mathrm{H}), 7.55(\mathrm{t}, J=7.4 \mathrm{~Hz}, 1 \mathrm{H}), 7.46(\mathrm{t}, J=7.7 \mathrm{~Hz}, 2 \mathrm{H}), 7.11(\mathrm{~d}, J=1.9 \mathrm{~Hz}, 1 \mathrm{H}), 7.06$ (dd, $J=7.9,2.1 \mathrm{~Hz}, 1 \mathrm{H}), 6.98(\mathrm{~d}, J=8.0 \mathrm{~Hz}, 1 \mathrm{H}$ ), 3.05-2.99 (multiple peaks, 3H), 2.94-2.86 (m, 1H), 2.83-2.75 (m, 2H), $2.68(\mathrm{~d}, J=12.2 \mathrm{~Hz}, 1 \mathrm{H}), 2.55-2.46(\mathrm{~m}, 2 \mathrm{H}), 2.40-2.25$ (multiple peaks, 2H), 2.00-1.90 (m, 2H), $1.30(\mathrm{~d}, J=7.2 \mathrm{~Hz}, 3 \mathrm{H}) .{ }^{13} \mathbf{C}$ NMR $\left(125 \mathrm{MHz}, \mathrm{CDCl}_{3}\right) \delta 200.3,147.7$, $140.1,137.3,133.0,131.9,130.6,128.7,128.2,126.0,125.8,62.1,58.4,55.0,38.4,36.3,35.8$, 22.0, 18.5. IR $\left(\mathrm{cm}^{-1}\right) 2935,1682,1596,1448,1364,1211,1120$. HRMS (ESI) $\mathrm{m} / z$ calc'd for $\left[\mathrm{C}_{21} \mathrm{H}_{24} \mathrm{ClNOH}\right]^{+} 342.1619$, found 342.1625 .

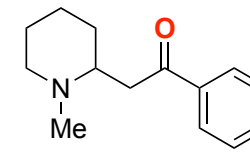

(23a): Eluted with $\mathrm{Et}_{3} \mathrm{~N} / \mathrm{EtOAc} / \mathrm{hexanes}(1: 10: 10)$ to give a clear oil $(26 \mathrm{mg}, 48 \%$ yield). ${ }^{1} \mathbf{H}$ NMR $\left(400 \mathrm{MHz}, \mathrm{CDCl}_{3}\right) \delta$ 7.97-7.92 (m, 2H), $7.55(\mathrm{t}, J=7.4 \mathrm{~Hz}, 1 \mathrm{H}), 7.49-7.41(\mathrm{~m}$, 2H), $3.34(\mathrm{dd}, J=16.1,4.9 \mathrm{~Hz}, 1 \mathrm{H}), 2.86(\mathrm{dd}, J=16.1,7.1 \mathrm{~Hz}, 1 \mathrm{H}), 2.82-2.70$ (multiple peaks, 2H), 2.25 (s, 3H), 2.23-2.14 (m, 1H), 1.74-1.52 (multiple peaks, 4H), 1.40-1.26 (m, 2H). ${ }^{13} \mathbf{C}$ NMR $\left(100 \mathrm{MHz}, \mathrm{CDCl}_{3}\right) \delta 199.5,137.4,133.2,128.8,128.3,59.6,56.4,43.8,42.5,32.5,26.1,23.8$. IR $\left(\mathrm{cm}^{-1}\right) 3059,2932,1681,1596,1447,1372,1201$. HRMS (ESI) $\mathrm{m} / z$ calc'd for $\left[\mathrm{C}_{14} \mathrm{H}_{19} \mathrm{NOH}\right]^{+}$ 218.1539 , found 218.1536 .

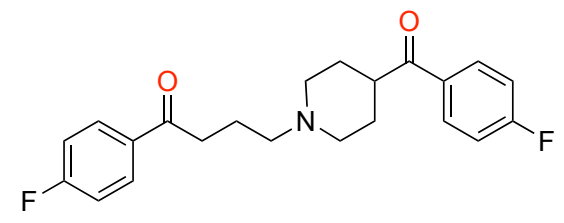

(24a): Synthesized on a $0.125 \mathrm{mmol}$ scale using $10 \mathrm{~mol} \%$ of $\mathrm{FeCl}_{3}$ and ${ }^{t} \mathrm{BuOOH}(0.92 \mathrm{~mL}, 6.8 \mathrm{mmol}, 54$ equiv) with slow addition over $24 \mathrm{~h}$. Total reaction time was 5 days. Crude residue was eluted with a $25-50 \%$ gradient of $\mathrm{Et}_{3} \mathrm{~N} / \mathrm{EtOAc} /$ hexanes (1:10:10) in hexanes to give a white solid (24 mg, 26\% yield, mp $\left.=131-133{ }^{\circ} \mathrm{C}\right) .{ }^{1} \mathbf{H}$ NMR (400 $\mathrm{MHz}, \mathrm{CDCl}_{3}$ ) $\delta 8.15-7.83$ (multiple peaks, 4H), 7.13 (multiple peaks, t, $J=8.8 \mathrm{~Hz}, 4 \mathrm{H}$ ), 3.24-3.11 (m, 1H), 3.06-2.92 (multiple peaks, 4H), $2.44(\mathrm{t}, J=7.0 \mathrm{~Hz}, 2 \mathrm{H}), 2.16-2.05(\mathrm{~m}, 2 \mathrm{H}), 1.95(\mathrm{p}, J=$ $7.1 \mathrm{~Hz}, 2 \mathrm{H}), 1.86-1.70$ (multiple peaks, 4H). ${ }^{13} \mathbf{C}$ NMR $\left(100 \mathrm{MHz}, \mathrm{CDCl}_{3}\right) \delta 201.2,198.8,165.8$ $\left(\mathrm{d}, J_{\mathrm{C}-\mathrm{F}}=254.3 \mathrm{~Hz}\right), 165.8\left(\mathrm{~d}, J_{\mathrm{C}-\mathrm{F}}=254.6 \mathrm{~Hz}\right), 133.2\left(\mathrm{~d}, J_{\mathrm{C}-\mathrm{F}}=114.9 \mathrm{~Hz}\right), 133.1\left(\mathrm{~d}, J_{\mathrm{C}-\mathrm{F}}=115.0\right.$ $\mathrm{Hz}), 131.0\left(\mathrm{~d}, J_{\mathrm{C}-\mathrm{F}}=9.2 \mathrm{~Hz}\right), 130.8\left(\mathrm{~d}, J_{\mathrm{C}-\mathrm{F}}=9.2 \mathrm{~Hz}\right), 115.9\left(\mathrm{~d}, J_{\mathrm{C}-\mathrm{F}}=21.8 \mathrm{~Hz}\right), 115.8\left(\mathrm{~d}, J_{\mathrm{C}-\mathrm{F}}=\right.$ $21.8 \mathrm{~Hz}), 57.9,53.2,43.8,36.3,28.8,21.8 .{ }^{19} \mathbf{F}$ NMR $\left(377 \mathrm{MHz}, \mathrm{CDCl}_{3}\right) \delta-105.6,-105.7$. IR $\left(\mathrm{cm}^{-1}\right)$ 3059, 2805, 1671, 1595, 1505, 1409, 1361, 1203. HRMS (ESI) $\mathrm{m} / z$ calc for $\left[\mathrm{C}_{22} \mathrm{H}_{23} \mathrm{~F}_{2} \mathrm{NO}_{2} \mathrm{H}\right]^{+} 372.1770$, found 372.1767 . 


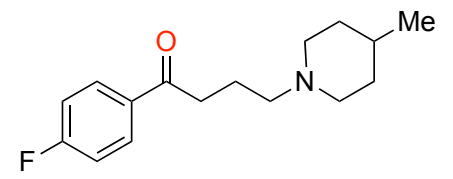

(25a): Synthesized on a $0.125 \mathrm{mmol}$ scale and eluted with $\mathrm{Et}_{3} \mathrm{~N} / \mathrm{EtOAc} /$ hexanes $(1: 10: 10)$ to give a white solid (17 mg, 52\% yield). ${ }^{1} \mathbf{H}$ NMR (400 MHz, $\left.\mathrm{CDCl}_{3}\right) \delta 8.04-7.93(\mathrm{~m}, 2 \mathrm{H}), 7.17-7.06(\mathrm{~m}, 2 \mathrm{H}), 2.95$ (t, $\left.J=7.2 \mathrm{~Hz}, 2 \mathrm{H}\right), 2.84$ (br. d, $J=11.6, \mathrm{~Hz}$, 2H), $2.36(\mathrm{t}, J=7.2 \mathrm{~Hz}, 2 \mathrm{H}), 1.98-1.85$ (multiple peaks, 4H), 1.61-1.53 (m, 2H), 1.40-1.22 (m, 1H), $1.20-1.08(\mathrm{~m}, 2 \mathrm{H}), 0.88(\mathrm{~d}, J=6.5 \mathrm{~Hz}, 3 \mathrm{H}) .{ }^{13} \mathbf{C} \mathbf{N M R}\left(100 \mathrm{MHz}, \mathrm{CDCl}_{3}\right) \delta 198.7,165.7(\mathrm{~d}$, $\left.J_{\mathrm{C}-\mathrm{F}}=254.2 \mathrm{~Hz}\right), 133.8\left(\mathrm{~d}, J_{\mathrm{C}-\mathrm{F}}=3.2 \mathrm{~Hz}\right), 130.8\left(\mathrm{~d}, J_{\mathrm{C}-\mathrm{F}}=9.2 \mathrm{~Hz}\right), 115.7\left(\mathrm{~d}, J_{\mathrm{C}-\mathrm{F}}=21.8 \mathrm{~Hz}\right), 58.3$, 54.1, 36.5, 34.4, 31.0, 22.1, 22.1. ${ }^{19}$ F NMR $\left(377 \mathrm{MHz}, \mathrm{CDCl}_{3}\right) \delta-105.9$. IR $\left(\mathrm{cm}^{-1}\right) 2946,1679$, $1596,1507,1454,1361,1224,1126$. HRMS (ESI) $\mathrm{m} / z$ calc for $\left[\mathrm{C}_{16} \mathrm{H}_{22} \mathrm{FNOH}\right]^{+} 264.1758$, found 264.1753.

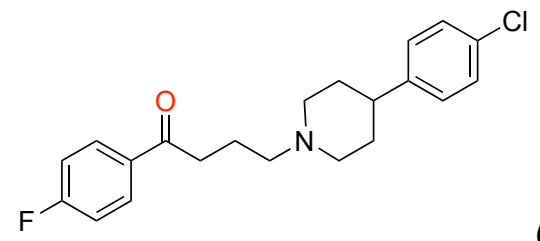

(26a): Synthesized on a $0.125 \mathrm{mmol}$ scale and eluted on a step gradient with $25 \%$ of $\mathrm{Et}_{3} \mathrm{~N} / \mathrm{EtOAc} /$ hexanes $(1: 10: 10)$ in hexanes (6 column volumes $(\mathrm{CV})$ ) and $50 \%$ of $\mathrm{Et}_{3} \mathrm{~N} / \mathrm{EtOAc} /$ hexanes $(1: 10: 10)$ in hexanes $(10 \mathrm{CV})$ to give a white solid $(18 \mathrm{mg}, 41 \%$ yield). ${ }^{1} \mathbf{H}$ NMR (400 MHz, $\left.\mathrm{CDCl}_{3}\right) \delta$ 8.05-7.99 (m, 2H), 7.26-7.22 (m, 2H), 7.16-7.08 (multiple peaks, 4H), 3.07-2.99 (m, 2H), $2.98(\mathrm{t}, J=7.1 \mathrm{~Hz}, 2 \mathrm{H}), 2.44$ (t, $J=7.1 \mathrm{~Hz}, 2 \mathrm{H}), 2.50-2.39$ (m, $1 \mathrm{H}), 2.10-2.00(\mathrm{~m}, 2 \mathrm{H}), 1.97(\mathrm{p}, J=7.1 \mathrm{~Hz}, 2 \mathrm{H}), 1.82-1.71(\mathrm{~m}, 2 \mathrm{H}), 1.71-1.58(\mathrm{~m}, 2 \mathrm{H}) .{ }^{13} \mathbf{C} \mathbf{N M R}$ $\left(100 \mathrm{MHz}, \mathrm{CDCl}_{3}\right) \delta 198.5,165.8\left(\mathrm{~d}, J_{\mathrm{C}-\mathrm{F}}=254.3 \mathrm{~Hz}\right), 145.0,133.8\left(\mathrm{~d}, J_{\mathrm{C}-\mathrm{F}}=3.2 \mathrm{~Hz}\right), 131.8$, $130.8\left(\mathrm{~d}, J_{\mathrm{C}-\mathrm{F}}=9.3 \mathrm{~Hz}\right), 128.6,128.3,115.7\left(\mathrm{~d}, J_{\mathrm{C}-\mathrm{F}}=21.8 \mathrm{~Hz}\right), 58.2,54.3,42.2,36.4,33.5,22.0$. ${ }^{19}$ F NMR $\left(377 \mathrm{MHz}, \mathrm{CDCl}_{3}\right) \delta-105.8$. IR $\left(\mathrm{cm}^{-1}\right)$ 2942, 1676, 1596, 1507, 1412, 1366, 1205. HRMS (ESI) $m / z$ calc for $\left[\mathrm{C}_{21} \mathrm{H}_{23} \mathrm{ClFNOH}\right]^{+} 360.1525$, found 360.1520 .

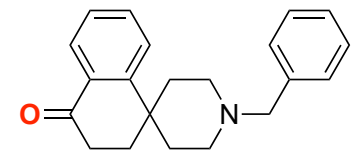

(27a): Reaction was run using a different amount of ${ }^{t} \mathrm{BuOOH}(0.21 \mathrm{~mL}$, 1.5 mmol, 6 equiv) with slow addition over $5 \mathrm{~h}$. Total reaction time was $24 \mathrm{~h}$. Crude residue was eluted with $20 \%$ of $\mathrm{Et}_{3} \mathrm{~N} / \mathrm{EtOAc} /$ hexanes $(1: 10: 10)$ in hexanes to give a clear oil $(47 \mathrm{mg}, 62 \%$ yield). ${ }^{1} \mathbf{H}$ NMR $\left(400 \mathrm{MHz}, \mathrm{CDCl}_{3}\right) \delta 7.93(\mathrm{dd}, J=7.8,1.5 \mathrm{~Hz}, 1 \mathrm{H}), 7.58-7.39(\mathrm{~m}, 2 \mathrm{H}), 7.31-7.06$ (multiple peaks, 6H), $3.50(\mathrm{~s}, 2 \mathrm{H}), 2.73$ (br. d, $J=11.8 \mathrm{~Hz}, 2 \mathrm{H}), 2.62-2.47(\mathrm{~m}, 2 \mathrm{H}), 2.26(\mathrm{td}, J=$ 12.2, $2.1 \mathrm{~Hz}, 2 \mathrm{H}), 2.15-2.05$ (multiple peaks, 4H), 1.68-1.52 (m, 2H). ${ }^{13} \mathbf{C}$ NMR (100 MHz, 
$\left.\mathrm{CDCl}_{3}\right) \delta 198.5,151.9,138.4,134.1,132.1,129.3,128.4,127.5,127.2,126.6,125.5,63.6,49.5$, 36.2, 35.5, 33.9, 28.4. IR $\left(\mathrm{cm}^{-1}\right)$ 3060, 2914, 1682, 1596, 1450, 1287, 1190. HRMS (ESI) $\mathrm{m} / z$ calc for $\left[\mathrm{C}_{21} \mathrm{H}_{23} \mathrm{NOH}\right]^{+} 306.1852$, found 306.1847 . 


\section{References}

(1) Howell, J. M.; Feng, K.; Clark, J. R.; Trzepkowski, L. J.; White, M. C. J. Am. Chem. Soc. 2015, 137, 14590.

(2) Clarke, H. T.; Gillespie, H. B.; Weisshaus, S. Z. J. Am. Chem. Soc. 1933, 55,4571 .

(3) Ward, R. S.; Davies, J.; Hodges, G.; Roberts, D. W. Synthesis 2002, 2002, 2431.

(4) Bosch, J.; Bonjoch, J.; Diez, A.; Linares, A.; Moral, M.; Rubiralta, M. Tetrahedron 1985, 41, 1753.

(5) Gülak, S.; Wu, L.; Liu, Q.; Franke, R.; Jackstell, R.; Beller, M. Angew. Chem. Int. Ed. 2014, 53, 7320.

(6) Chung, F.; Tisné, C.; Lecourt, T.; Dardel, F.; Micouin, L. Angew. Chem. Int. Ed. 2007, 46, 4489. 
IX. ${ }^{1} \mathrm{H}$ NMR, ${ }^{13} \mathrm{C}$ NMR and ${ }^{19}$ F NMR Spectra 
${ }^{1}$ H-NMR and ${ }^{13}$ C-NMR Spectra for compound 2
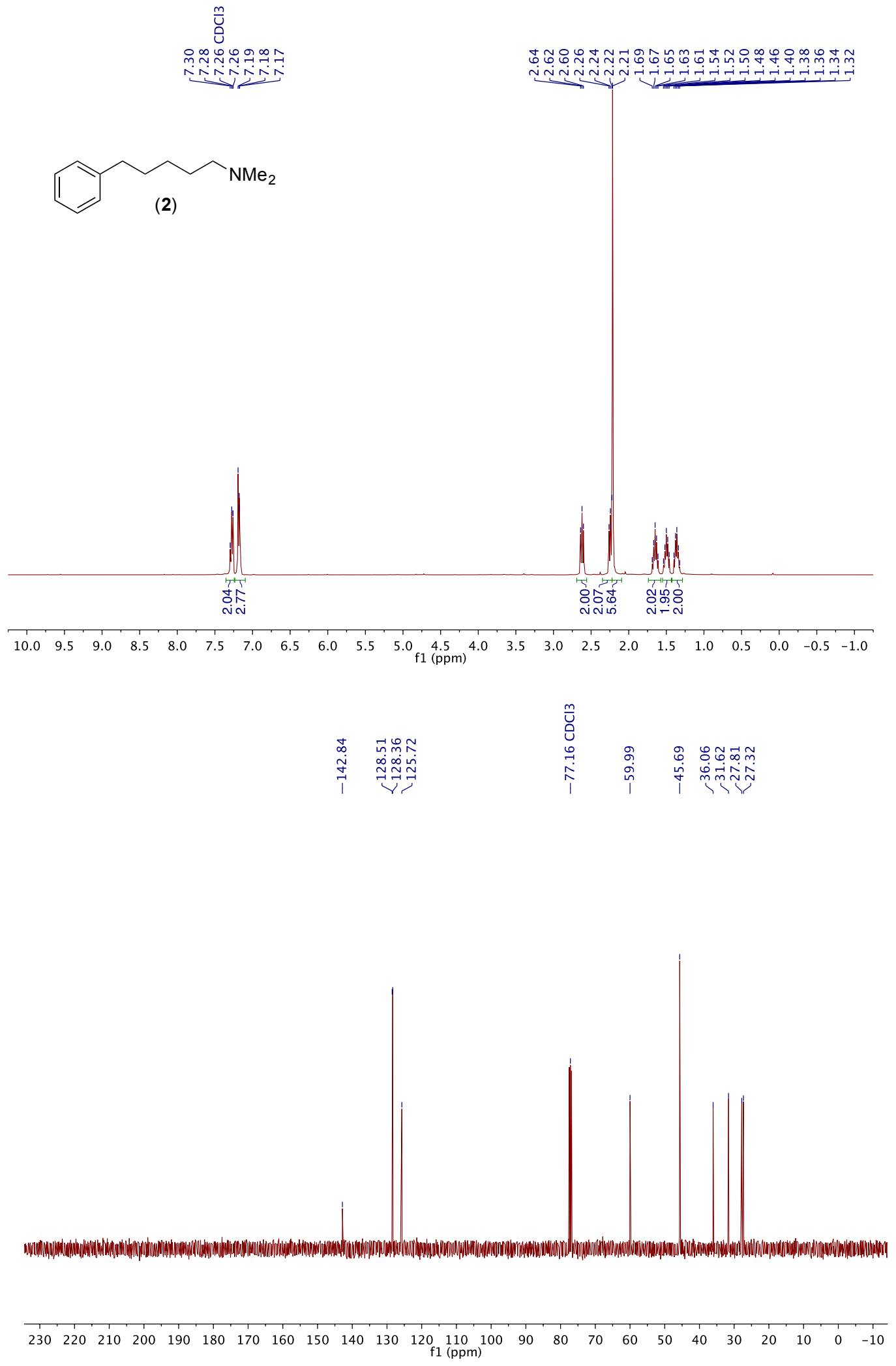
${ }^{1} \mathrm{H}-\mathrm{NMR}$ and ${ }^{13} \mathrm{C}-\mathrm{NMR}$ Spectra for compound 6
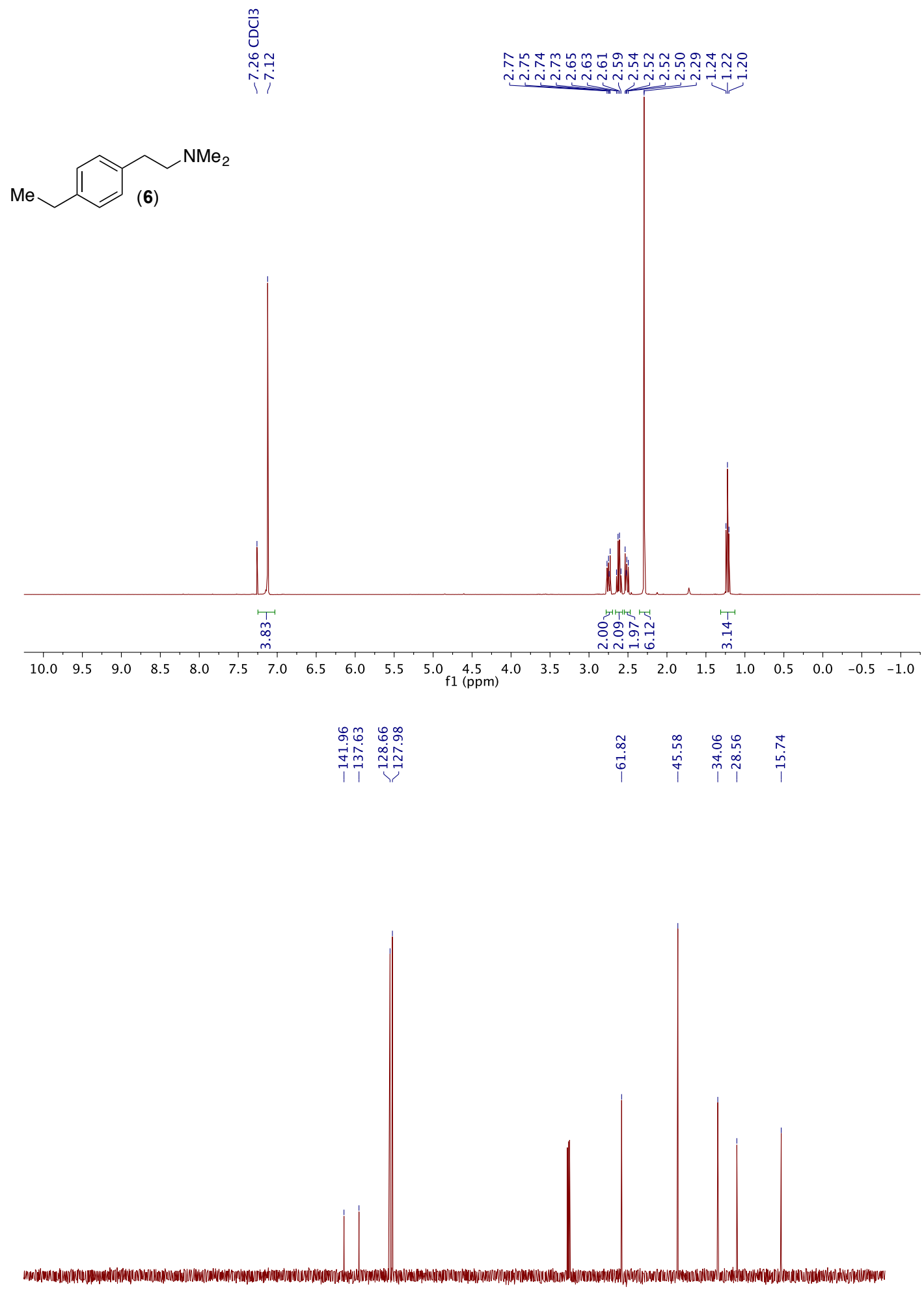

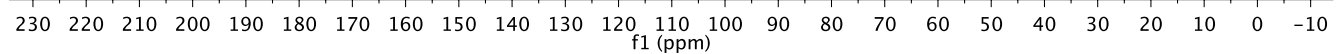




\section{${ }^{1} \mathrm{H}-\mathrm{NMR}$ and ${ }^{13} \mathrm{C}-\mathrm{NMR}$ Spectra for compound 7}

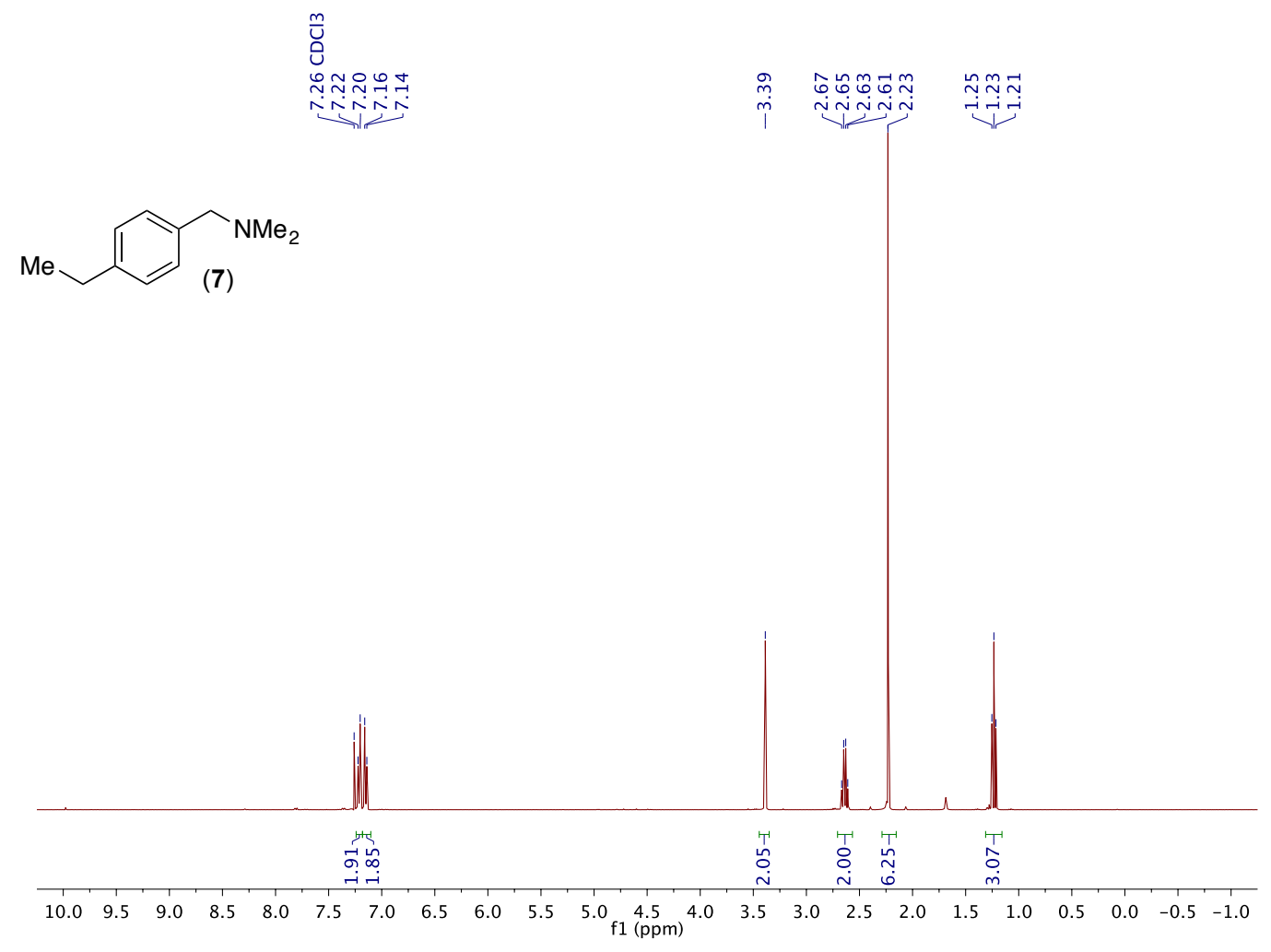

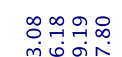

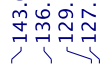
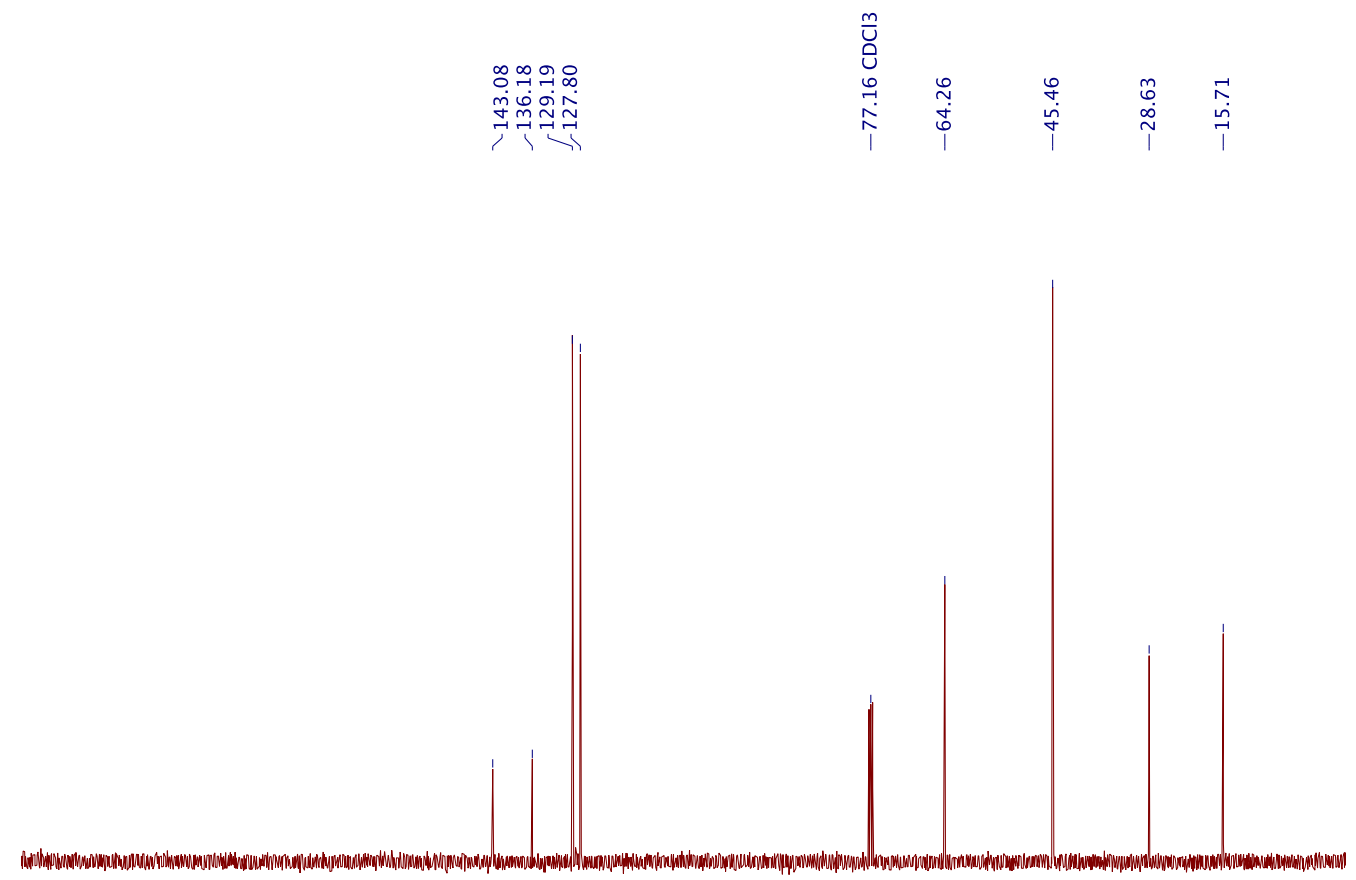

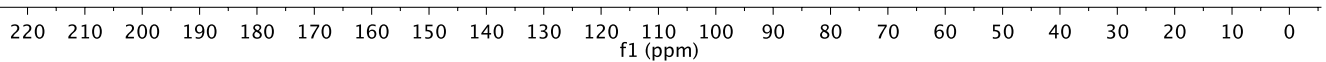


${ }^{1} \mathrm{H}-\mathrm{NMR}$ and ${ }^{13} \mathrm{C}-\mathrm{NMR}$ Spectra for compound 9
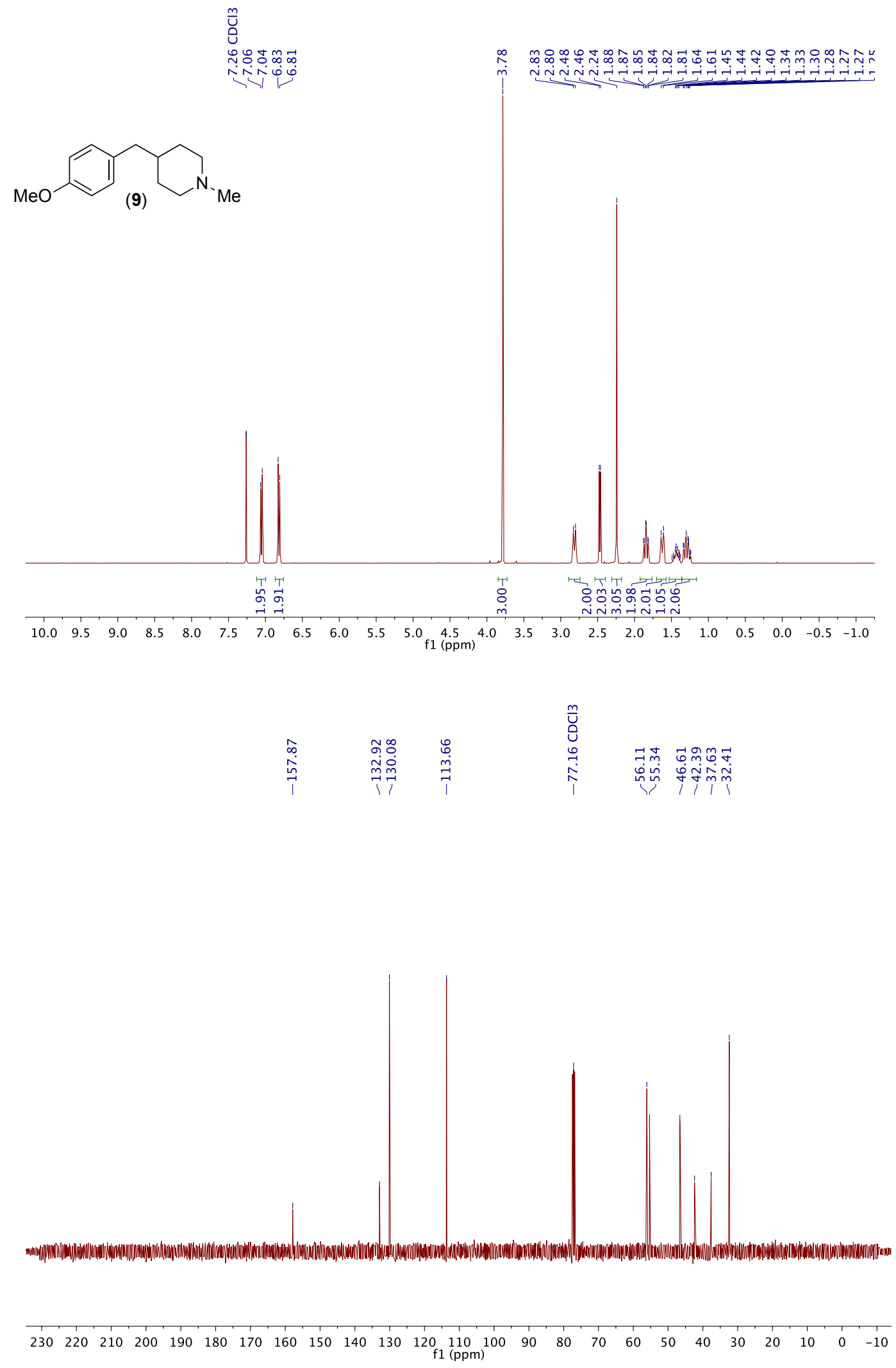
${ }^{1} \mathrm{H}-\mathrm{NMR}$ and ${ }^{13} \mathrm{C}-\mathrm{NMR}$ Spectra for compound $\mathbf{1 0}$
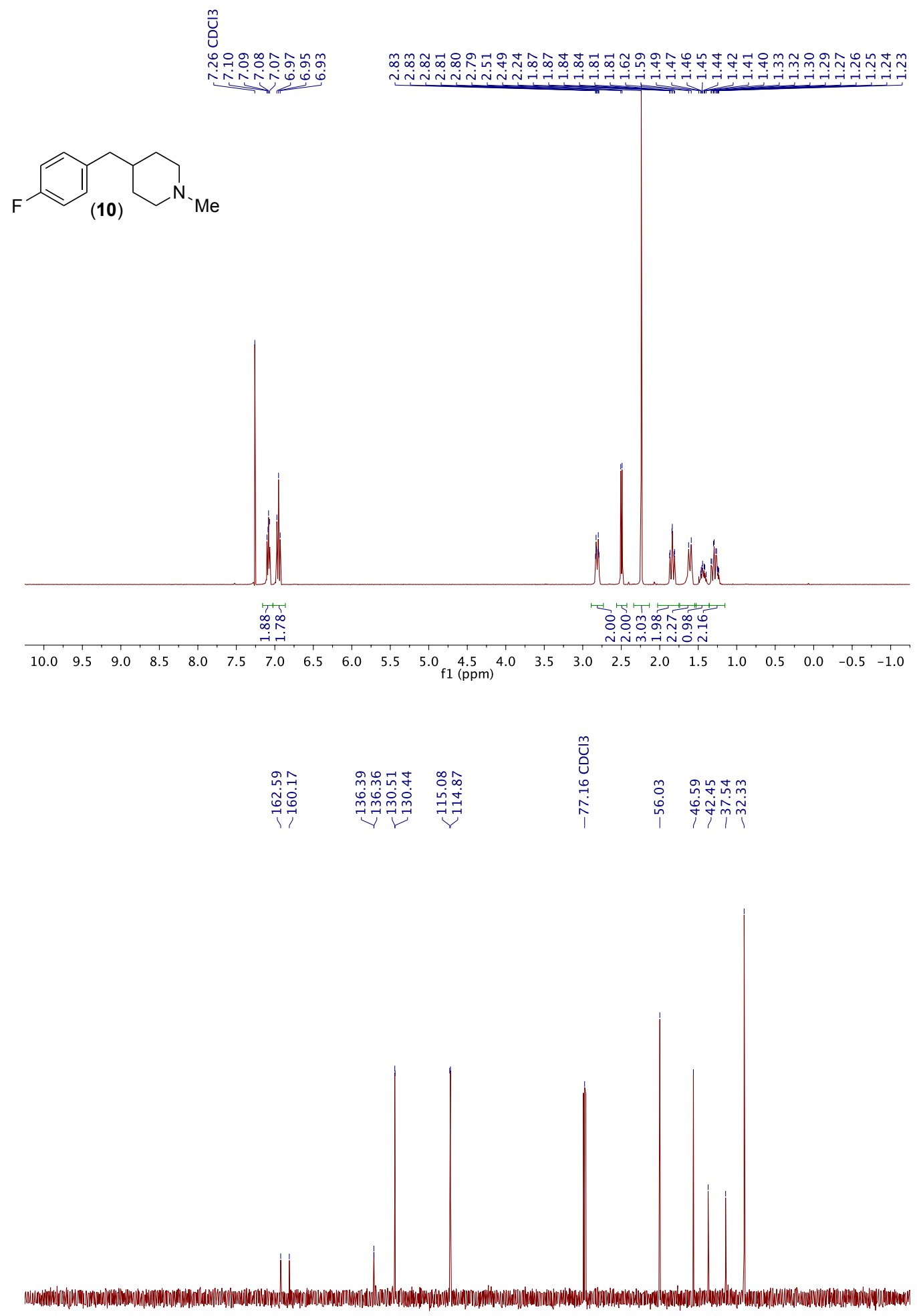

$\begin{array}{lllllllllllllllllllllllllll}230 & 220 & 210 & 200 & 190 & 180 & 170 & 160 & 150 & 140 & 130 & 120 & \mathrm{f} 1 \mathrm{lp}(\mathrm{pm}) & 100 & 90 & 80 & 70 & 60 & 50 & 40 & 30 & 20 & 10 & 0 & -10\end{array}$ 


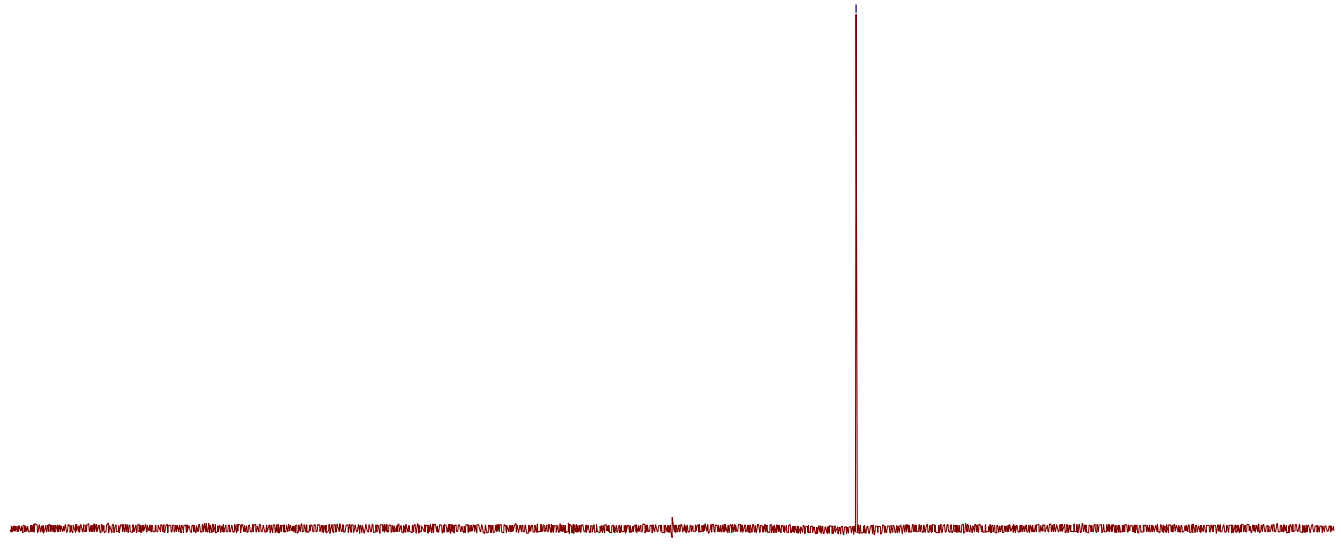

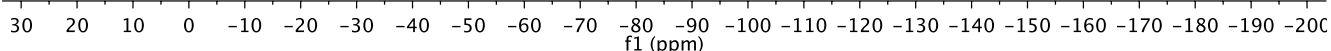

S-33 
${ }^{1} \mathrm{H}-\mathrm{NMR}$ and ${ }^{13} \mathrm{C}-\mathrm{NMR}$ Spectra for compound $\mathbf{1 1}$
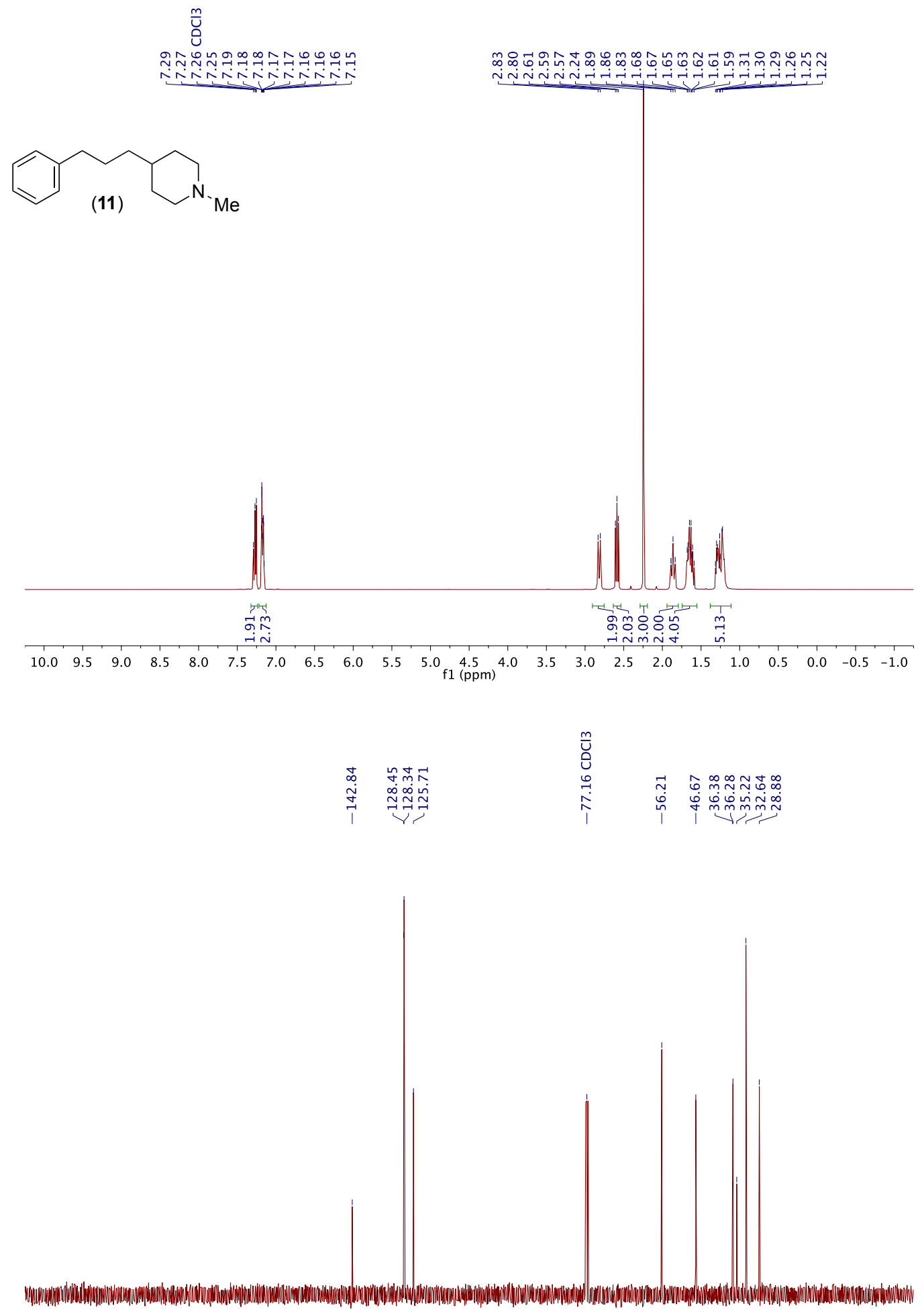

$\begin{array}{llllllllllllllllllllllllllllll}230 & 220 & 210 & 200 & 190 & 180 & 170 & 160 & 150 & 140 & 130 & 120 & 110 & 100 & 90 & 80 & 70 & 60 & 50 & 40 & 30 & 20 & 10 & 0 & -10\end{array}$ 
${ }^{1} \mathrm{H}-\mathrm{NMR}$ and ${ }^{13} \mathrm{C}-\mathrm{NMR}$ Spectra for compound $\mathbf{1 2}$
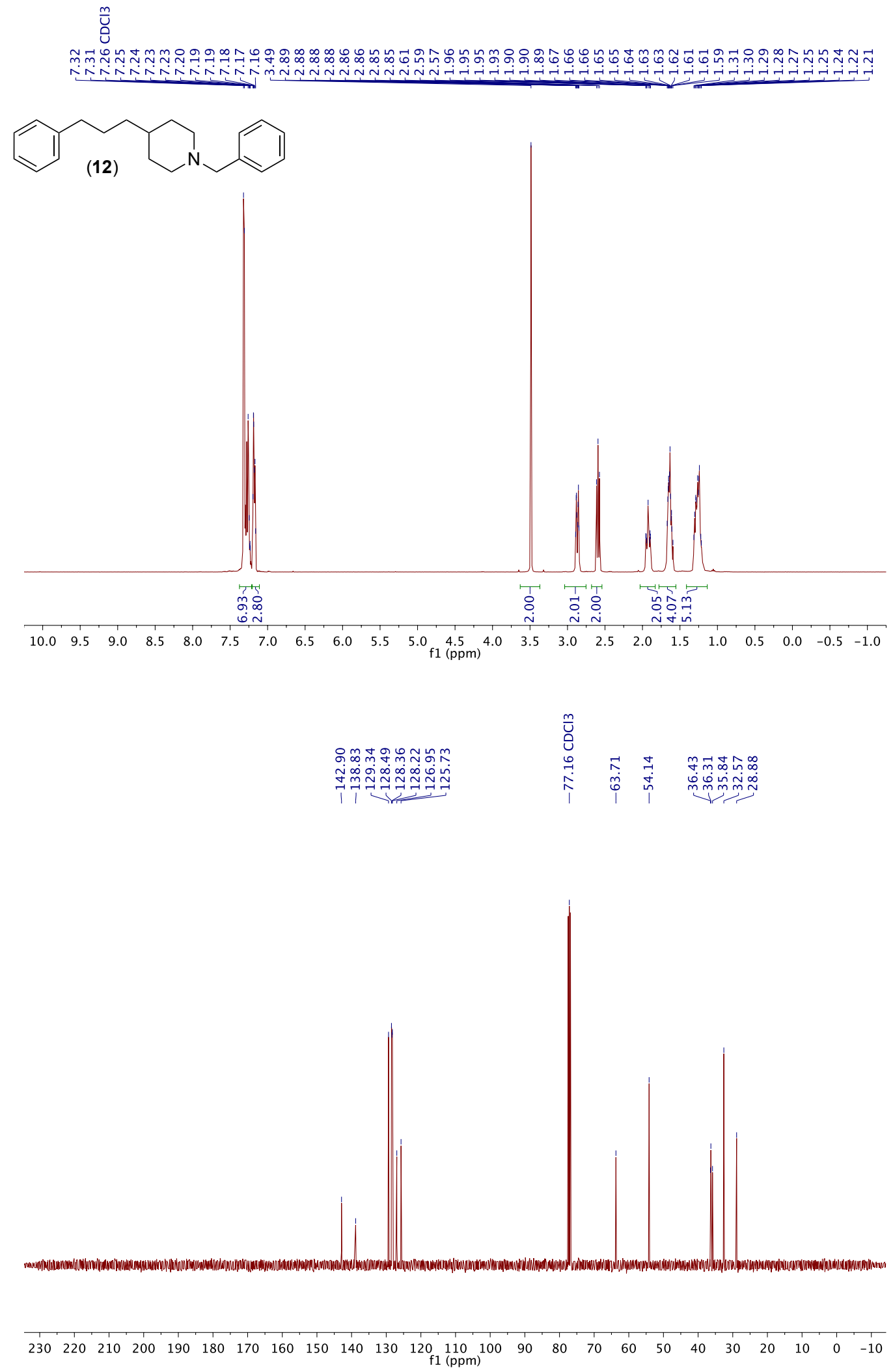
${ }^{1} \mathrm{H}-\mathrm{NMR}$ and ${ }^{13} \mathrm{C}-\mathrm{NMR}$ Spectra for compound $\mathbf{1 3}$
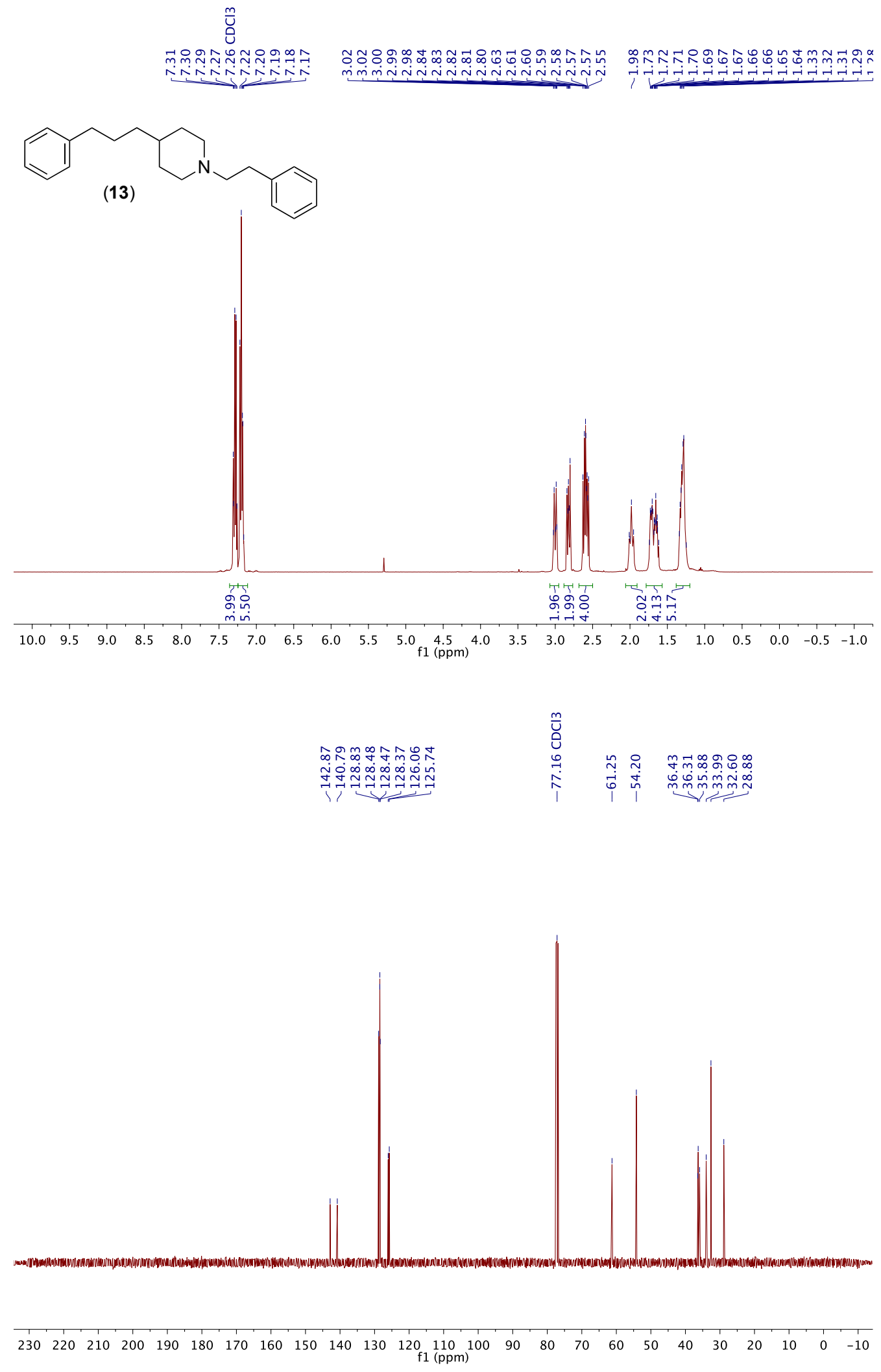
${ }^{1} \mathrm{H}-\mathrm{NMR}$ and ${ }^{13} \mathrm{C}-\mathrm{NMR}$ Spectra for compound $\mathbf{1 4}$
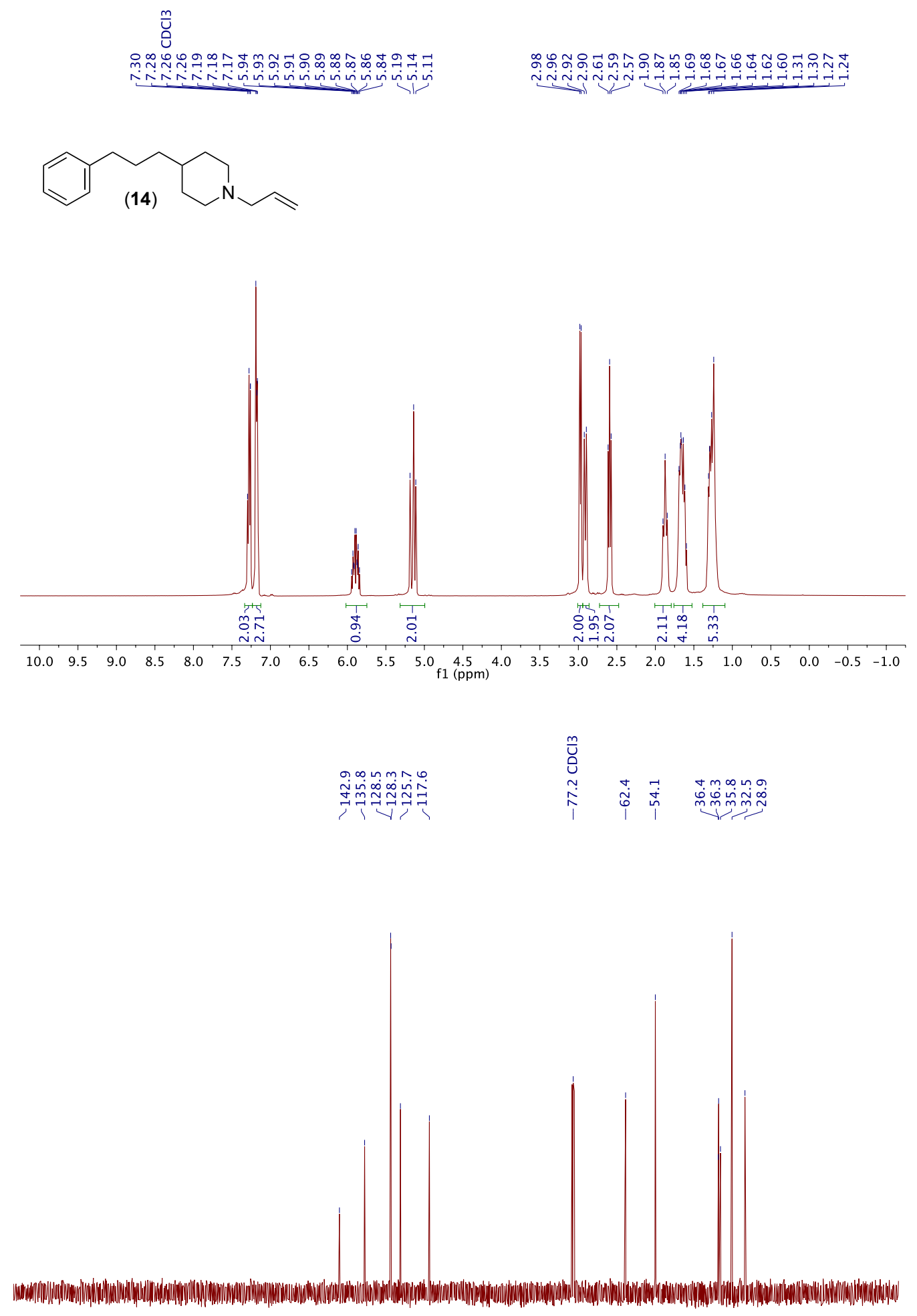

$\begin{array}{llllllllllllllllllllllllllllllllllll}230 & 220 & 210 & 200 & 190 & 180 & 170 & 160 & 150 & 140 & 130 & 120 & 110 & 100 & 90 & 80 & 70 & 60 & 50 & 40 & 30 & 20 & 10 & 0 & -10\end{array}$ 
${ }^{1} \mathrm{H}-\mathrm{NMR}$ and ${ }^{13} \mathrm{C}-\mathrm{NMR}$ Spectra for compound $\mathbf{1 5}$
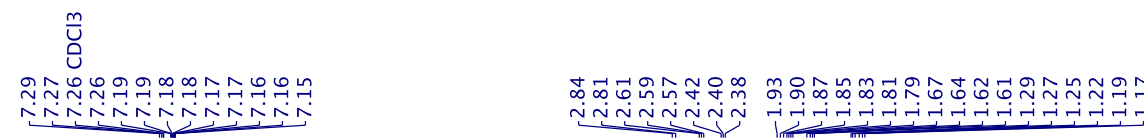

$\triangle(15)$
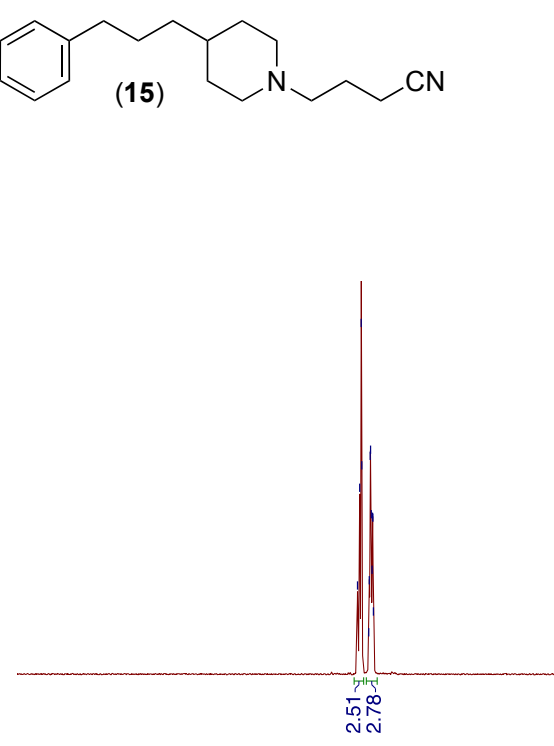

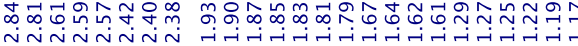

$\begin{array}{lllllllllllllllll}10.0 & 9.5 & 9.0 & 8.5 & 8.0 & 7.5 & 7.0 & 6.5 & 6.0 & 5.5 & 5.0 & 4.5 & 4.0 \\ \mathrm{f} 1(\mathrm{ppm}) & & & \end{array}$
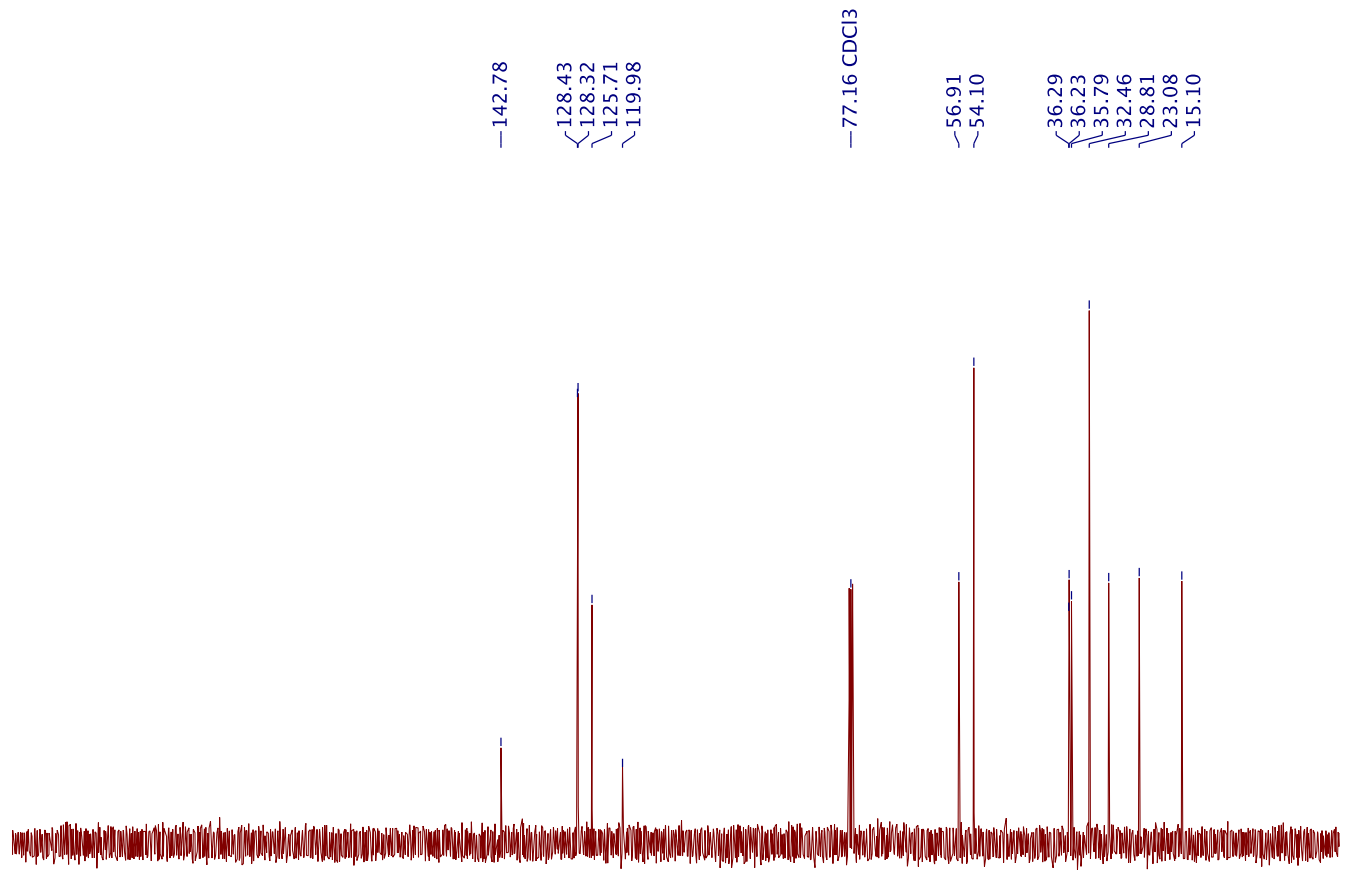

$\begin{array}{lllllllllllllllllllllllllllllllllll}230 & 220 & 210 & 200 & 190 & 180 & 170 & 160 & 150 & 140 & 130 & 120 & 110 & 100 & 90 & 80 & 70 & 60 & 50 & 40 & 30 & 20 & 10 & 0 & -10\end{array}$ 
${ }^{1} \mathrm{H}-\mathrm{NMR}$ and ${ }^{13} \mathrm{C}-\mathrm{NMR}$ Spectra for compound $\mathbf{1 6}$
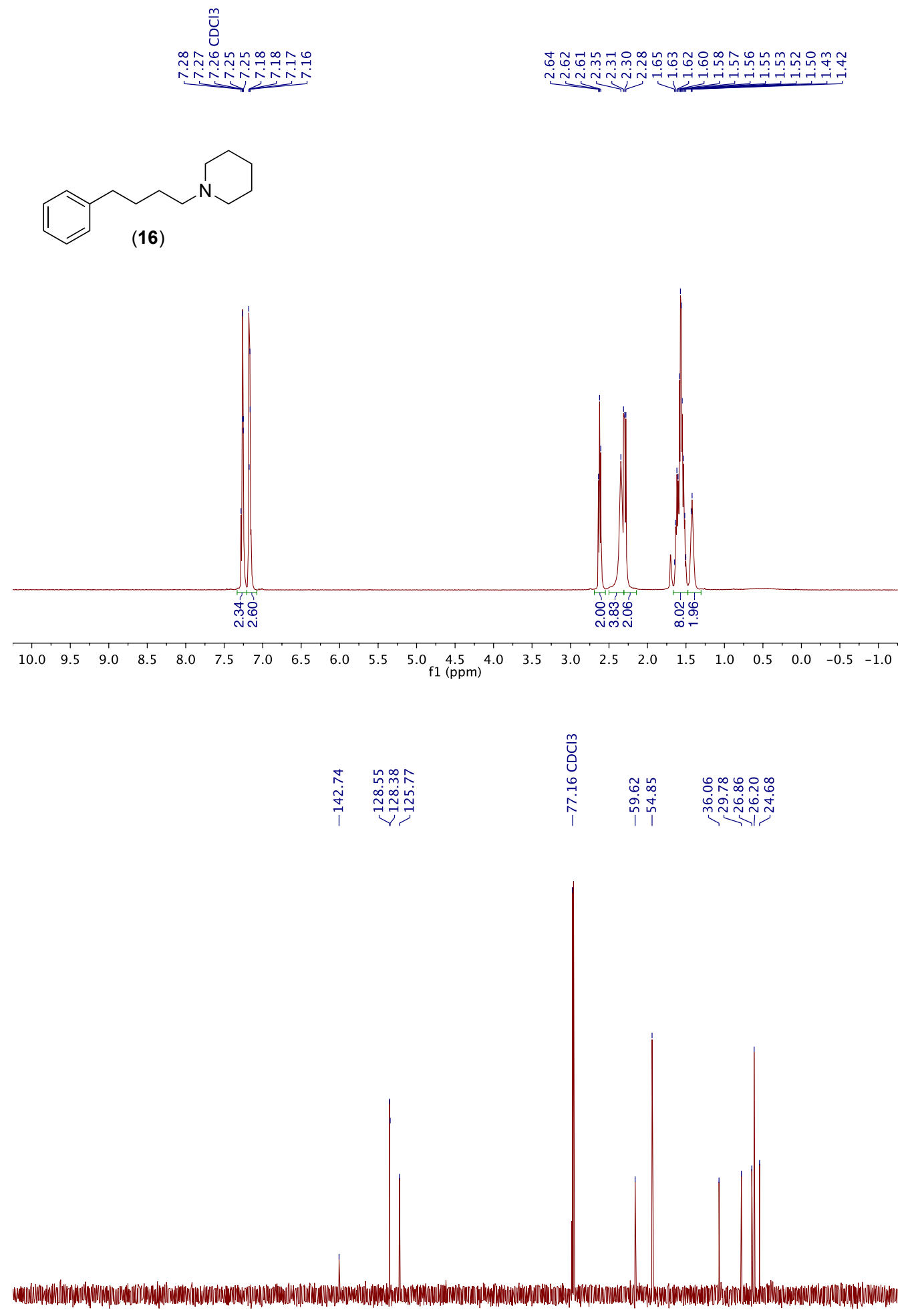

$\begin{array}{rlllllllllllllllllllllllllllll}230 & 220 & 210 & 200 & 190 & 180 & 170 & 160 & 150 & 140 & 130 & 120 & 110 & 100 & 90 & 80 & 70 & 60 & 50 & 40 & 30 & 20 & 10 & 0 & -10\end{array}$ 
${ }^{1} \mathrm{H}-\mathrm{NMR}$ and ${ }^{13} \mathrm{C}-\mathrm{NMR}$ Spectra for compound $\mathbf{1 7}$
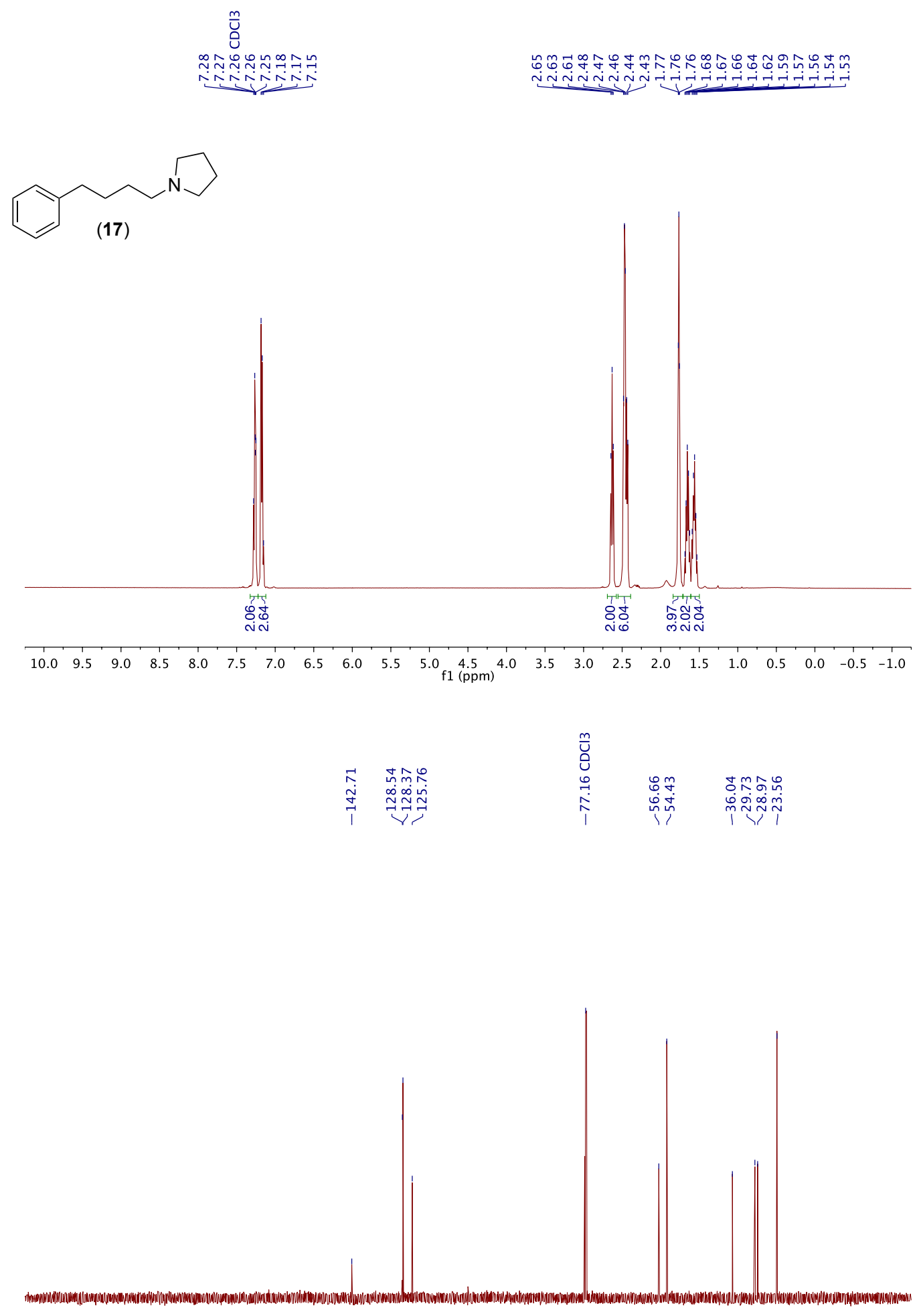

$\begin{array}{llllllllllllllllllllllllllllllll}230 & 220 & 210 & 200 & 190 & 180 & 170 & 160 & 150 & 140 & 130 & 120 & 110 & 100 & 90 & 80 & 70 & 60 & 50 & 40 & 30 & 20 & 10 & 0 & -10\end{array}$ 
${ }^{1} \mathrm{H}-\mathrm{NMR}$ and ${ }^{13} \mathrm{C}-\mathrm{NMR}$ Spectra for compound $\mathbf{1 8}$

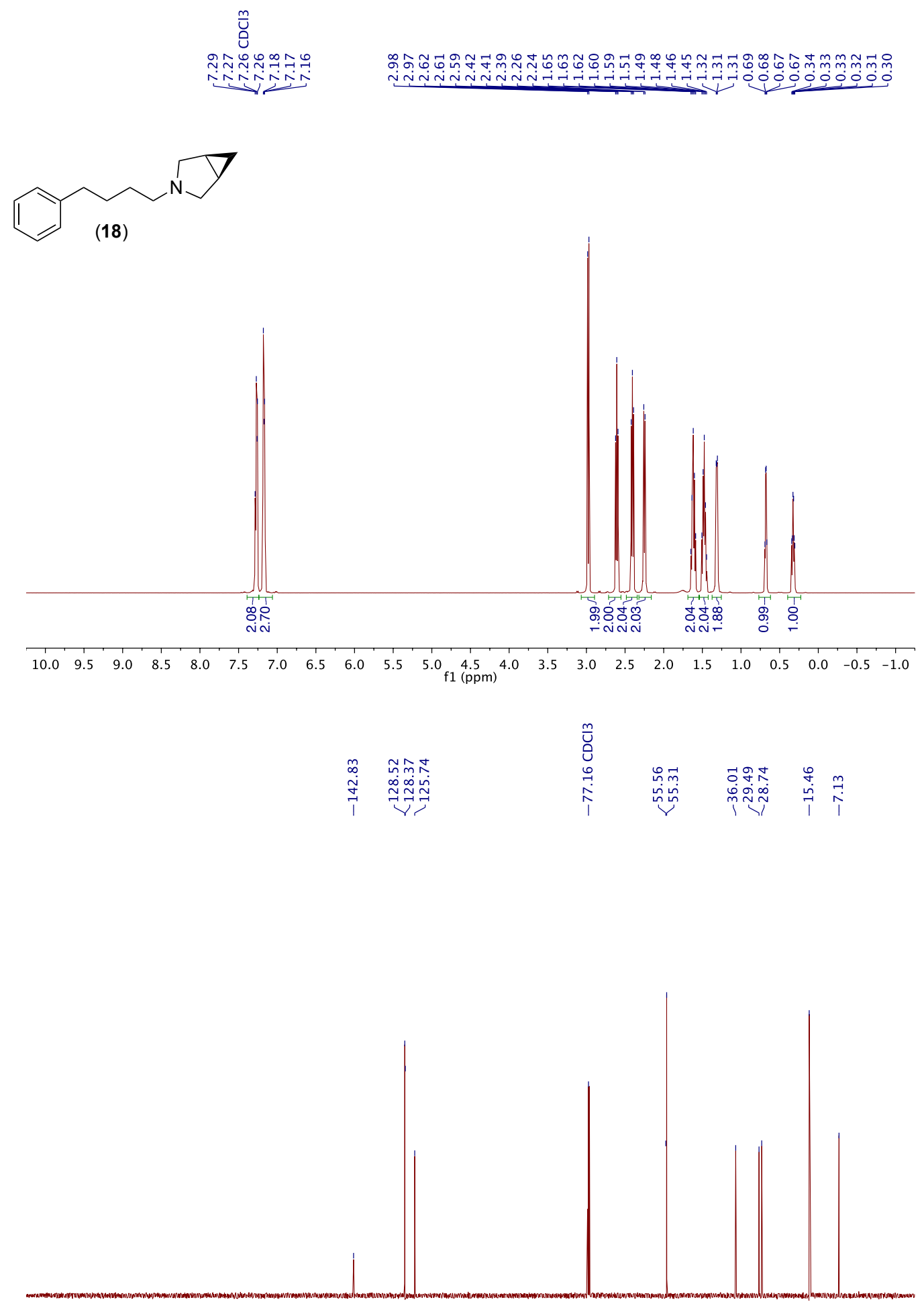

$\begin{array}{rlllllllllllllllllllllllllllll}230 & 220 & 210 & 200 & 190 & 180 & 170 & 160 & 150 & 140 & 130 & 120 & 110 & 100 & 90 & 80 & 70 & 60 & 50 & 40 & 30 & 20 & 10 & 0 & -10\end{array}$ 
${ }^{1} \mathrm{H}-\mathrm{NMR}$ and ${ }^{13} \mathrm{C}-\mathrm{NMR}$ Spectra for compound 19
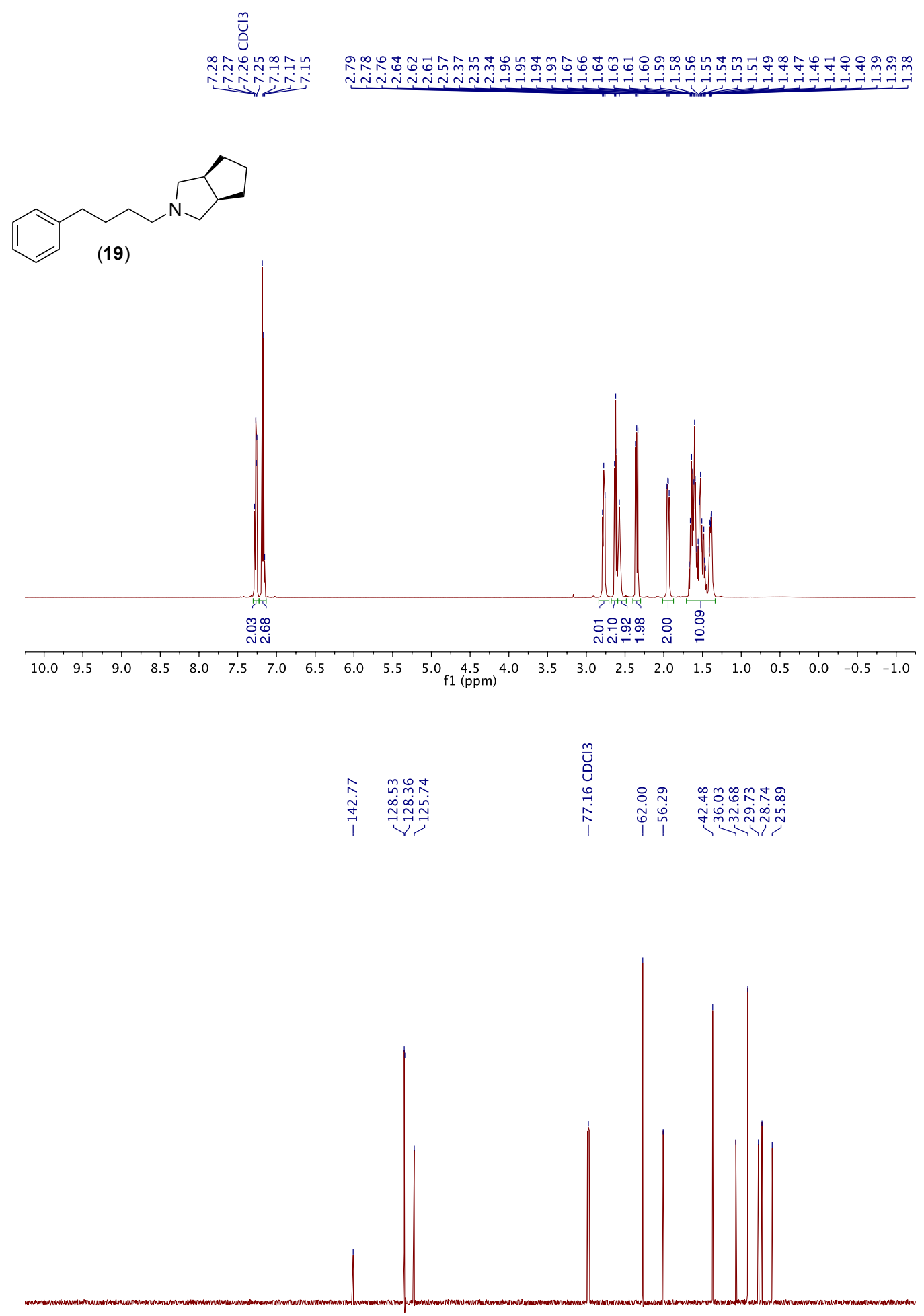

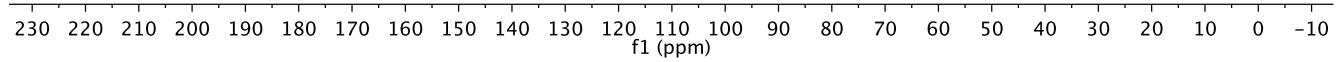


${ }^{1} \mathrm{H}-\mathrm{NMR}$ and ${ }^{13} \mathrm{C}-\mathrm{NMR}$ Spectra for compound $\mathbf{2 0}$
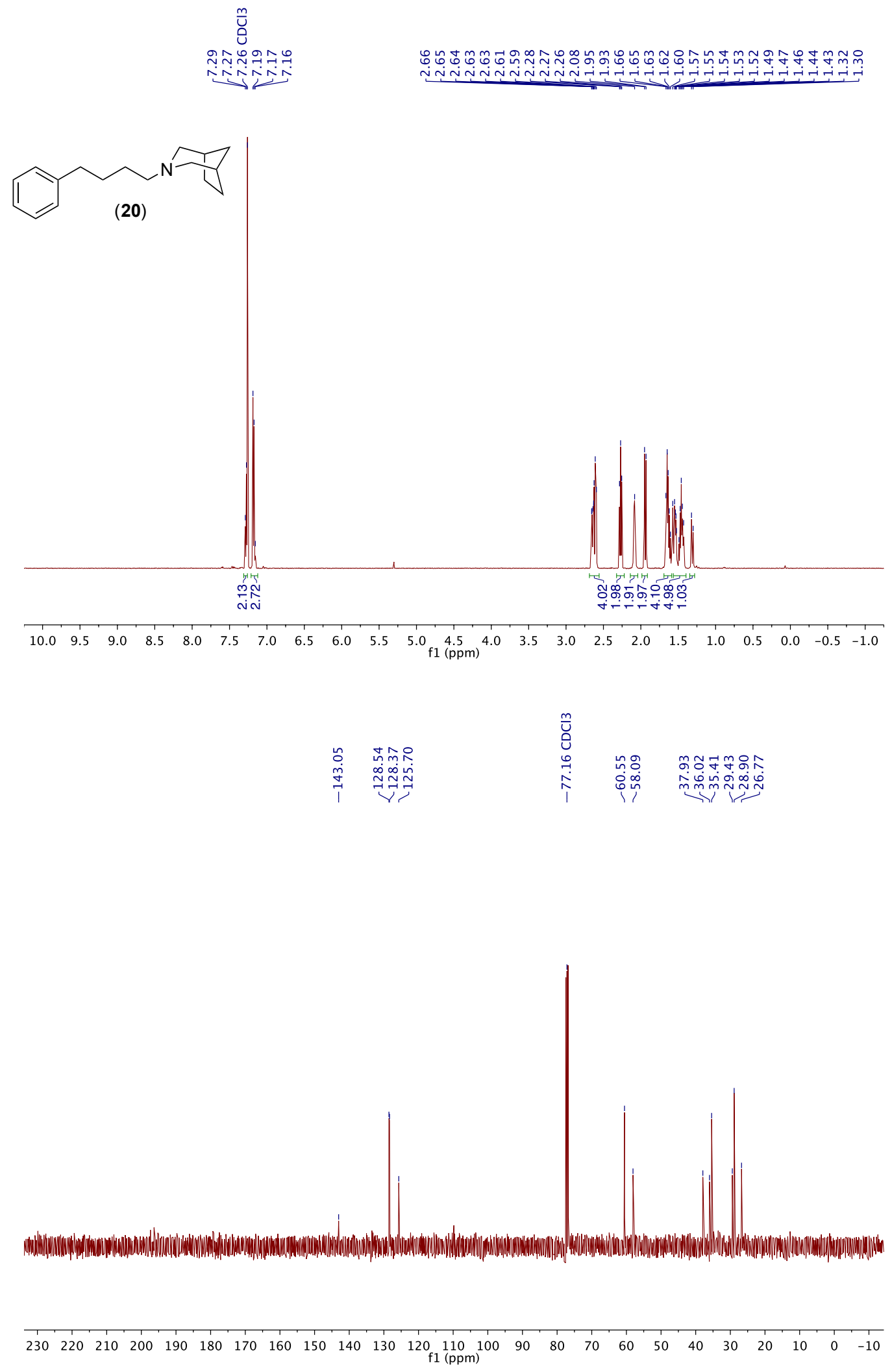
${ }^{1} \mathrm{H}-\mathrm{NMR}$ and ${ }^{13} \mathrm{C}-\mathrm{NMR}$ Spectra for compound 21
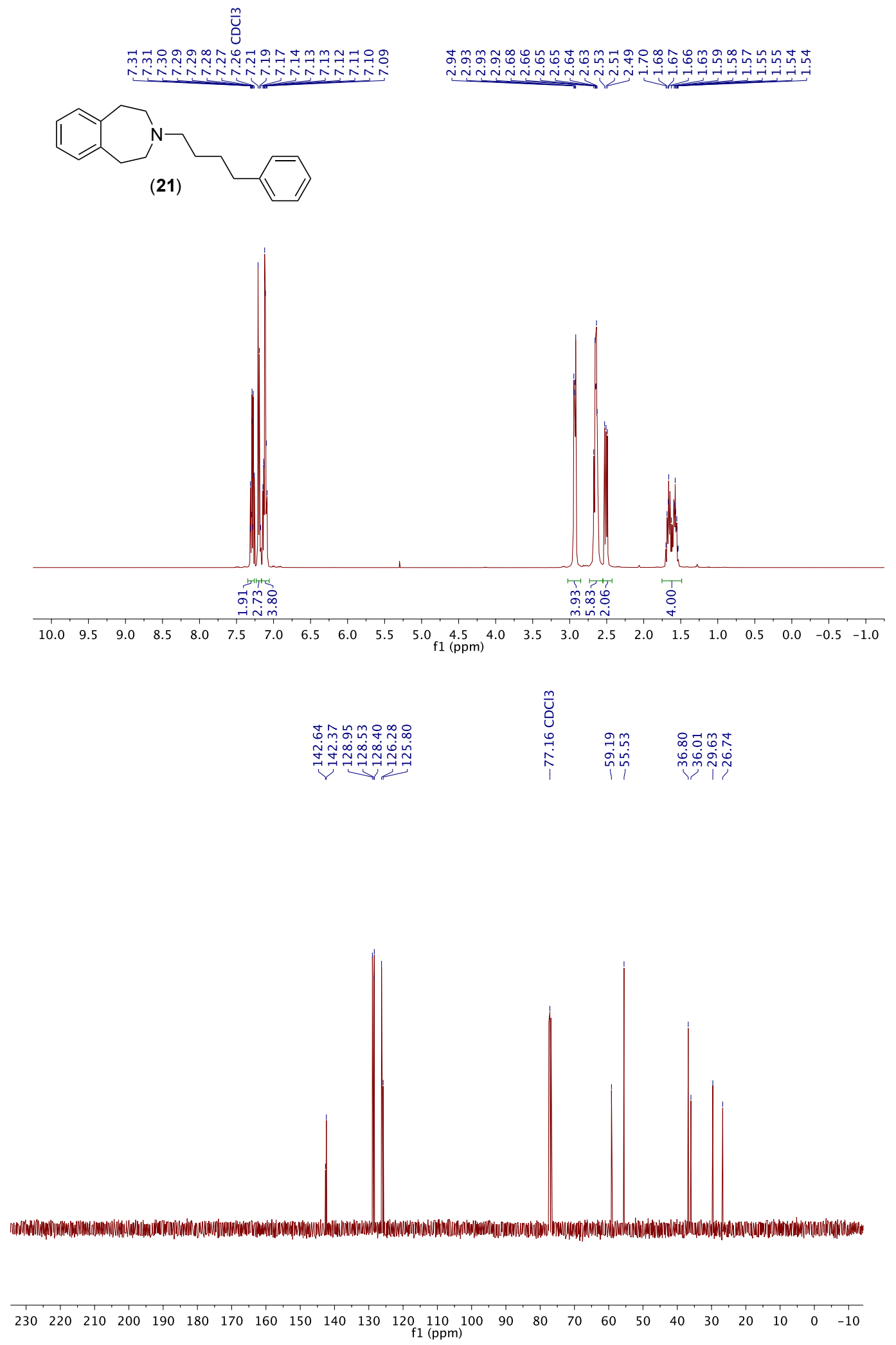
${ }^{1} \mathrm{H}-\mathrm{NMR}$ and ${ }^{13} \mathrm{C}-\mathrm{NMR}$ Spectra for compound $\mathbf{2 2}$

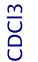

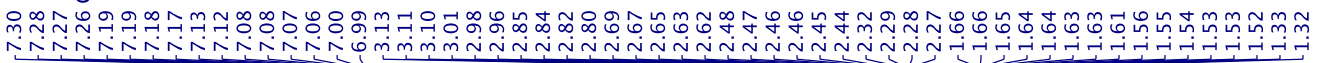

(22)

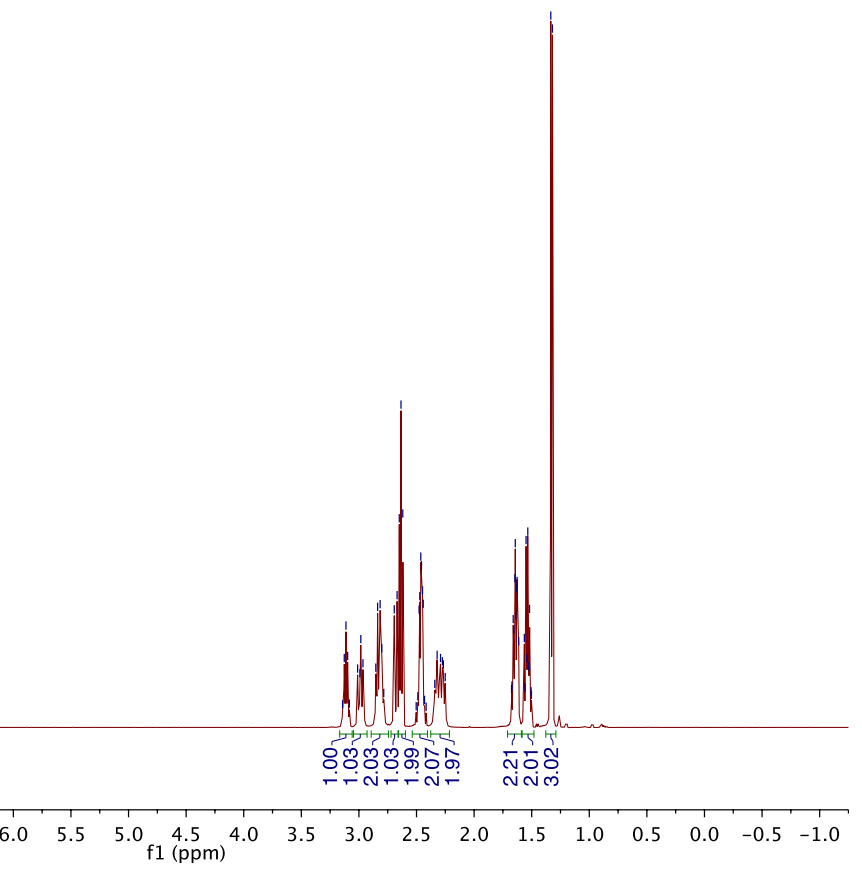

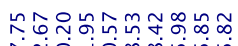

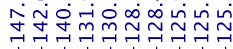
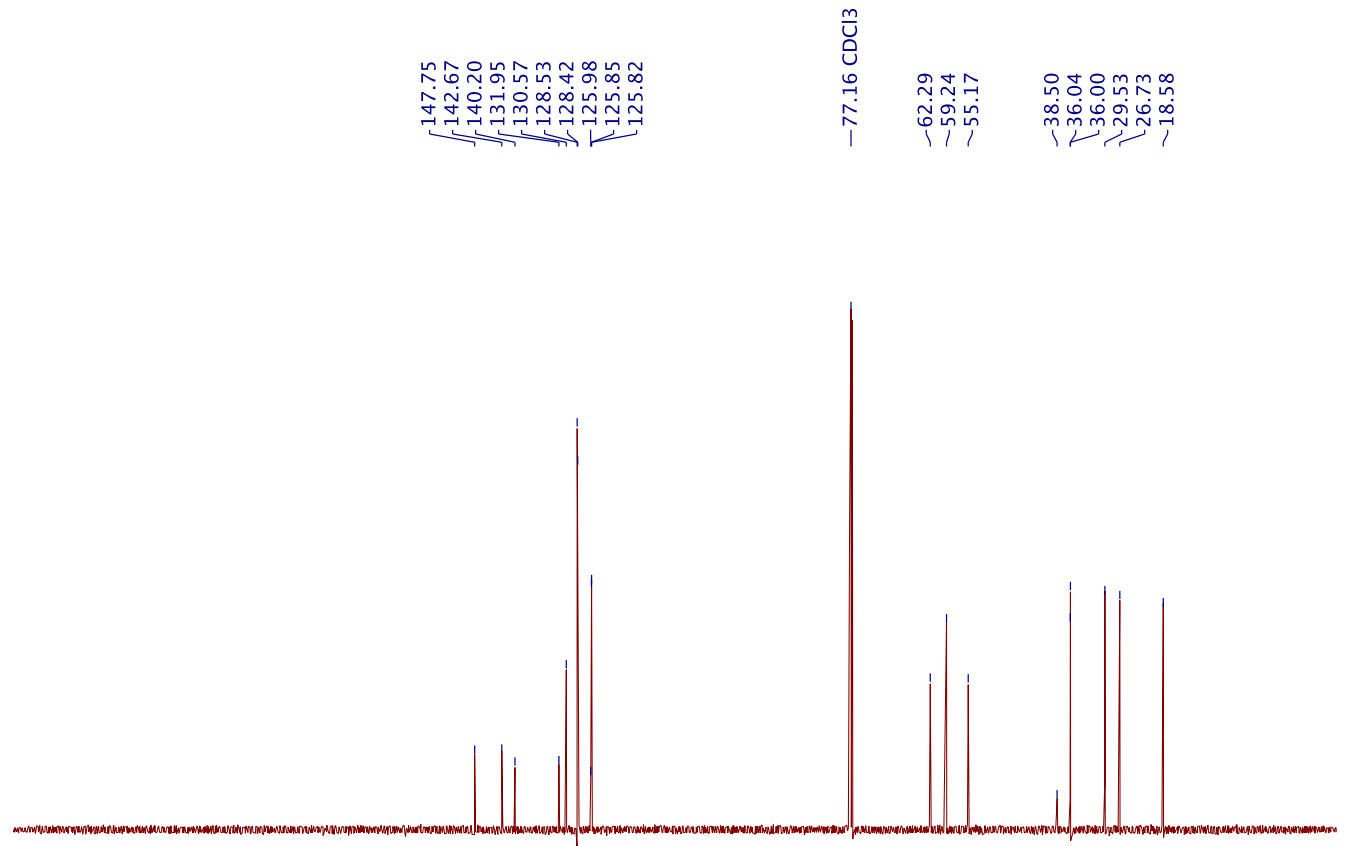

$\begin{array}{lllllllllllllllllllllllllllllllllllll}230 & 220 & 210 & 200 & 190 & 180 & 170 & 160 & 150 & 140 & 130 & 120 & 110 & 100 & 90 & 80 & 70 & 60 & 50 & 40 & 30 & 20 & 10 & 0 & -10\end{array}$ 
${ }^{1} \mathrm{H}-\mathrm{NMR}$ and ${ }^{13} \mathrm{C}-\mathrm{NMR}$ Spectra for compound $\mathbf{2 3}$

บับ

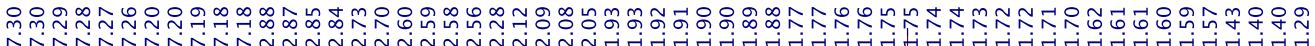

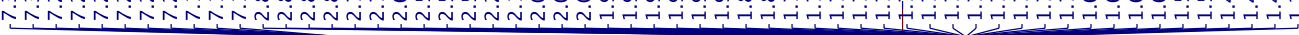
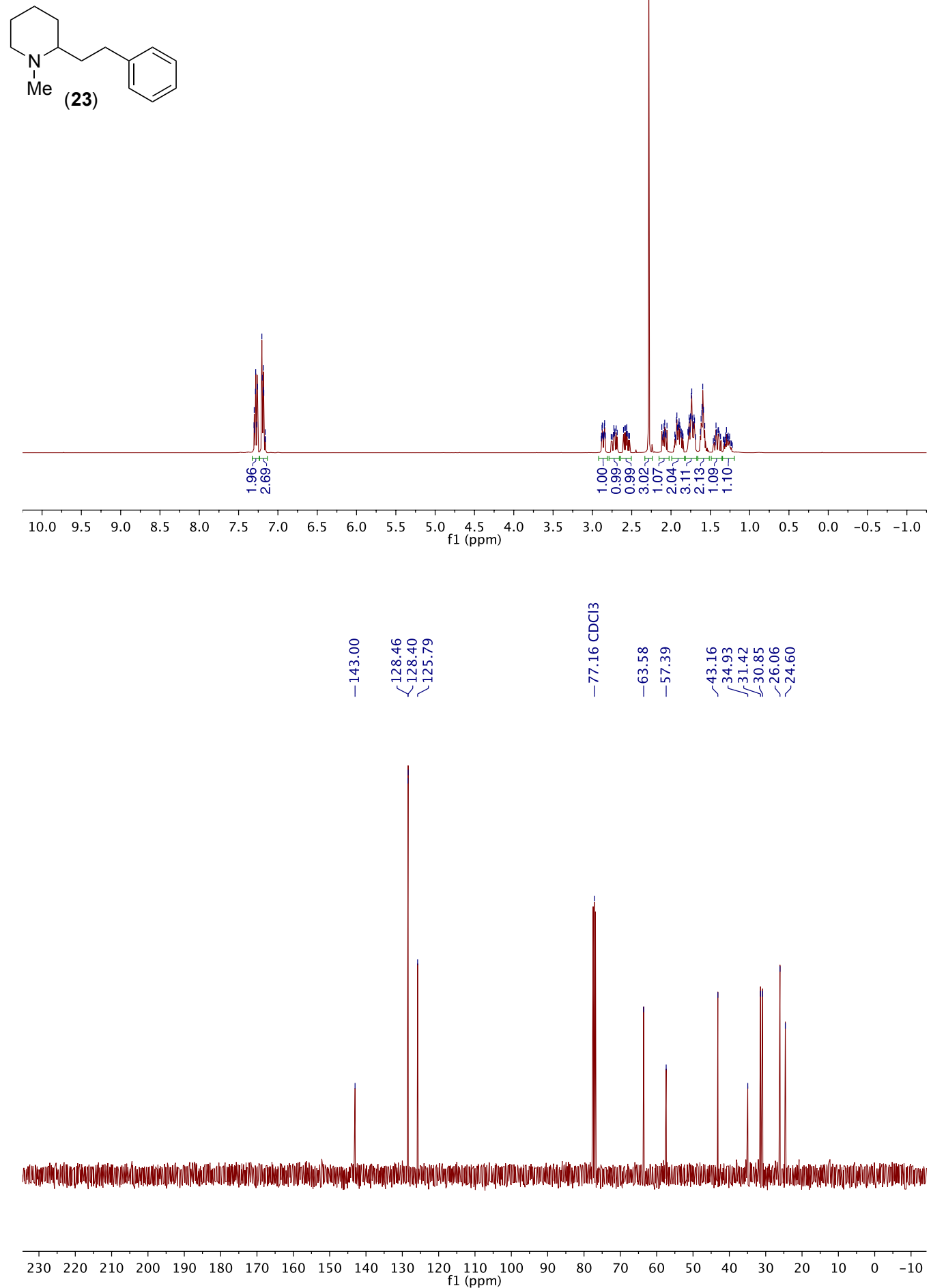
${ }^{1} \mathrm{H}-\mathrm{NMR}$ and ${ }^{13} \mathrm{C}-\mathrm{NMR}$ and ${ }^{19} \mathrm{~F}$-NMR Spectra for compound $\mathbf{2 4}$

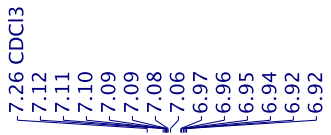

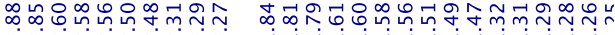

Ninitiviogogog

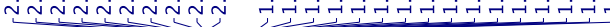
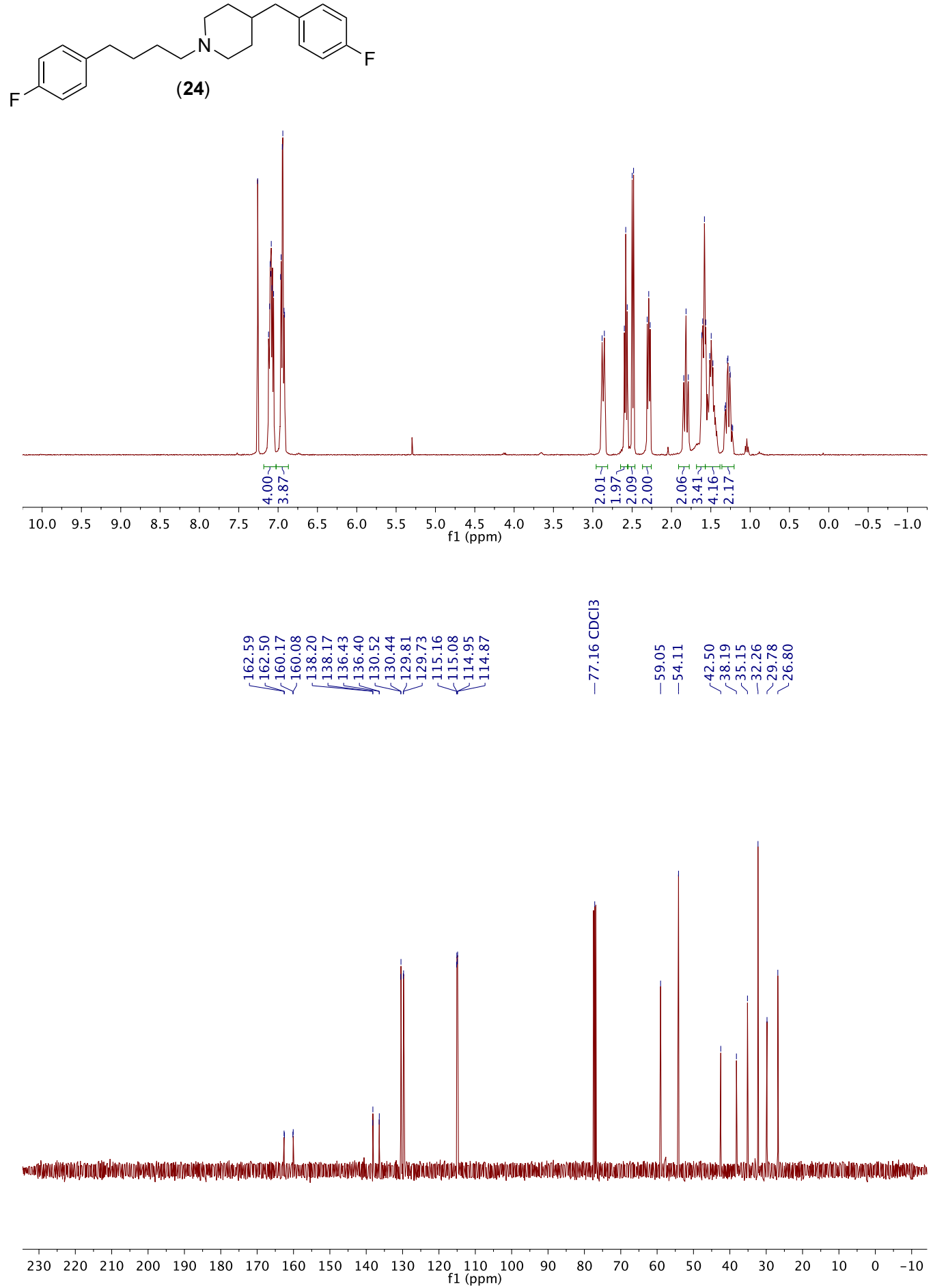


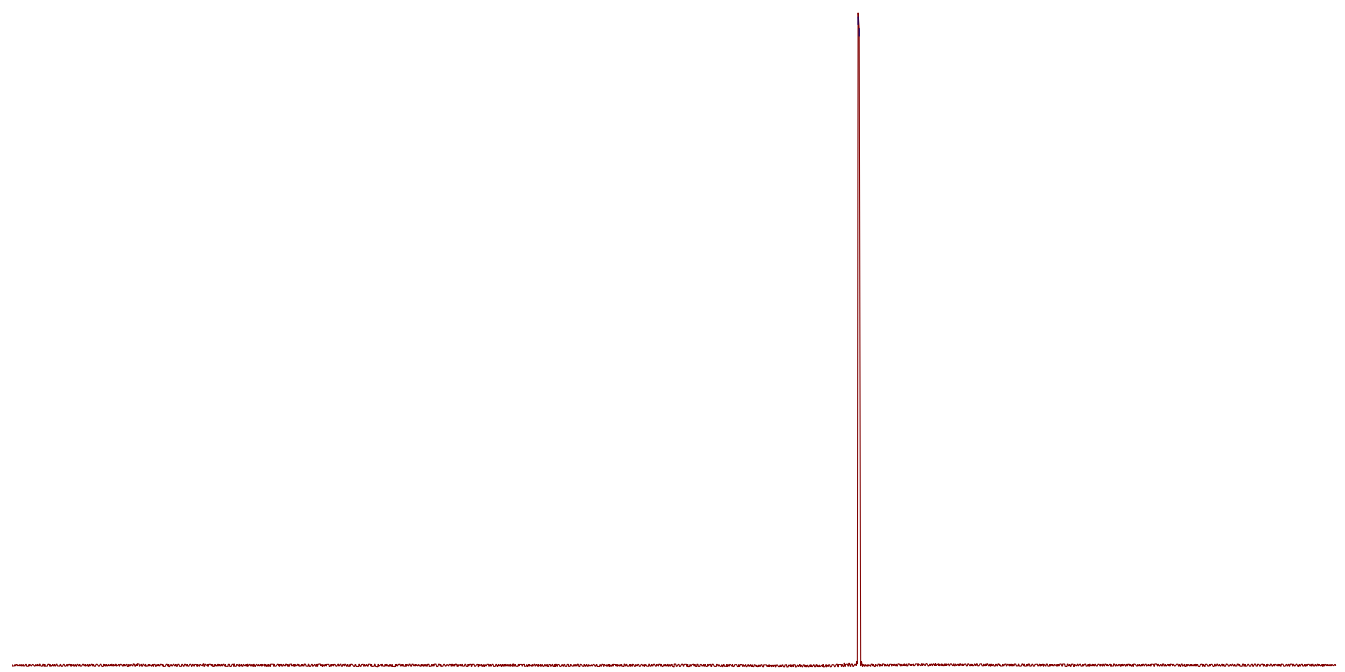

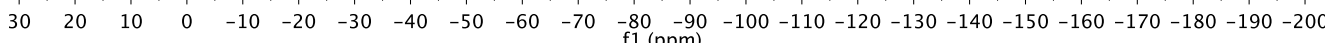


${ }^{1} \mathrm{H}-\mathrm{NMR}$ and ${ }^{13} \mathrm{C}-\mathrm{NMR}$ Spectra and ${ }^{19}$ F-NMR for compound $\mathbf{2 5}$

రับ

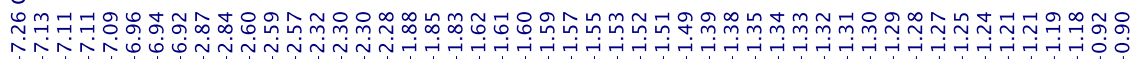<smiles>CC1CCN(CCCCc2ccc(F)cc2)CC1</smiles>
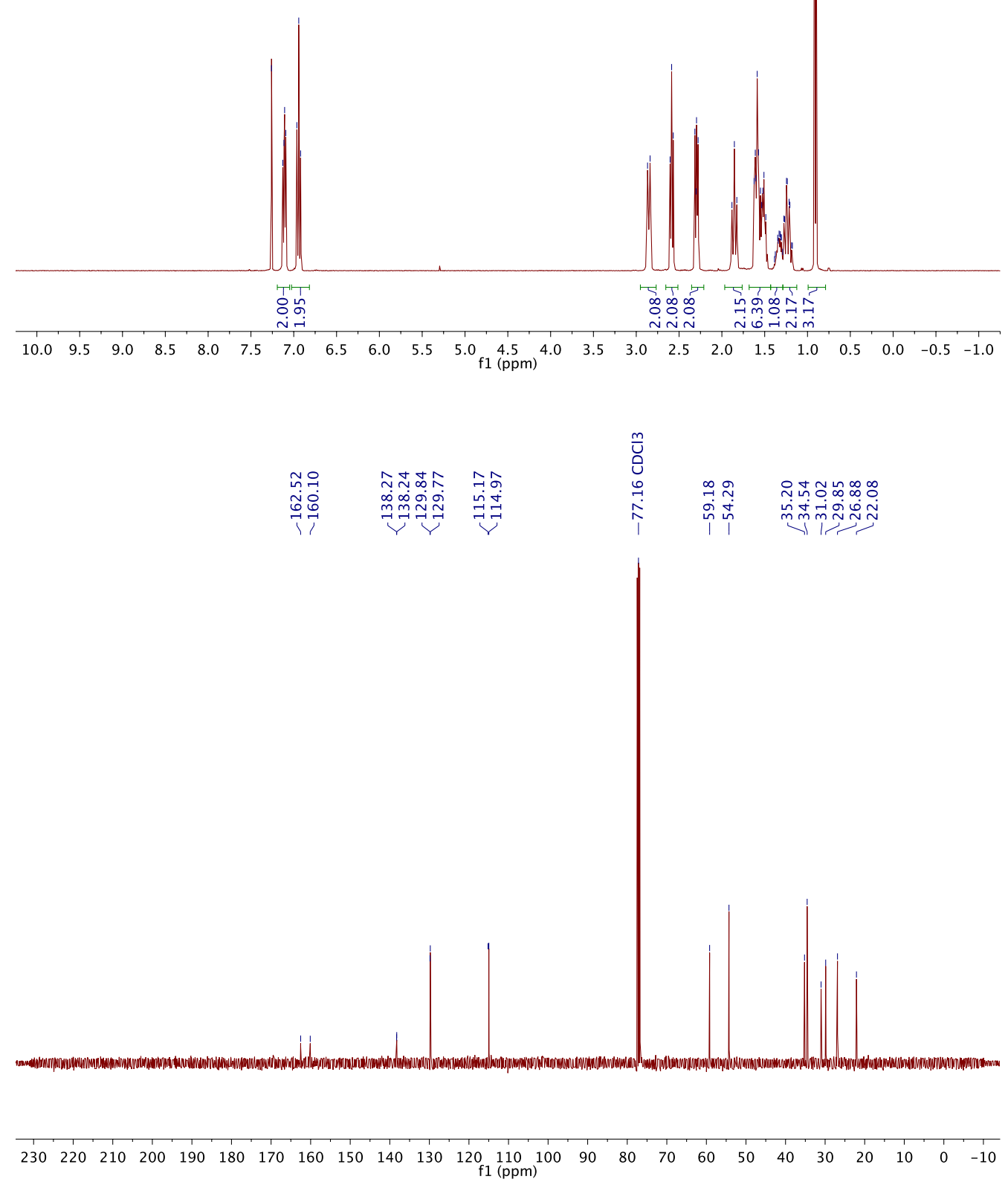


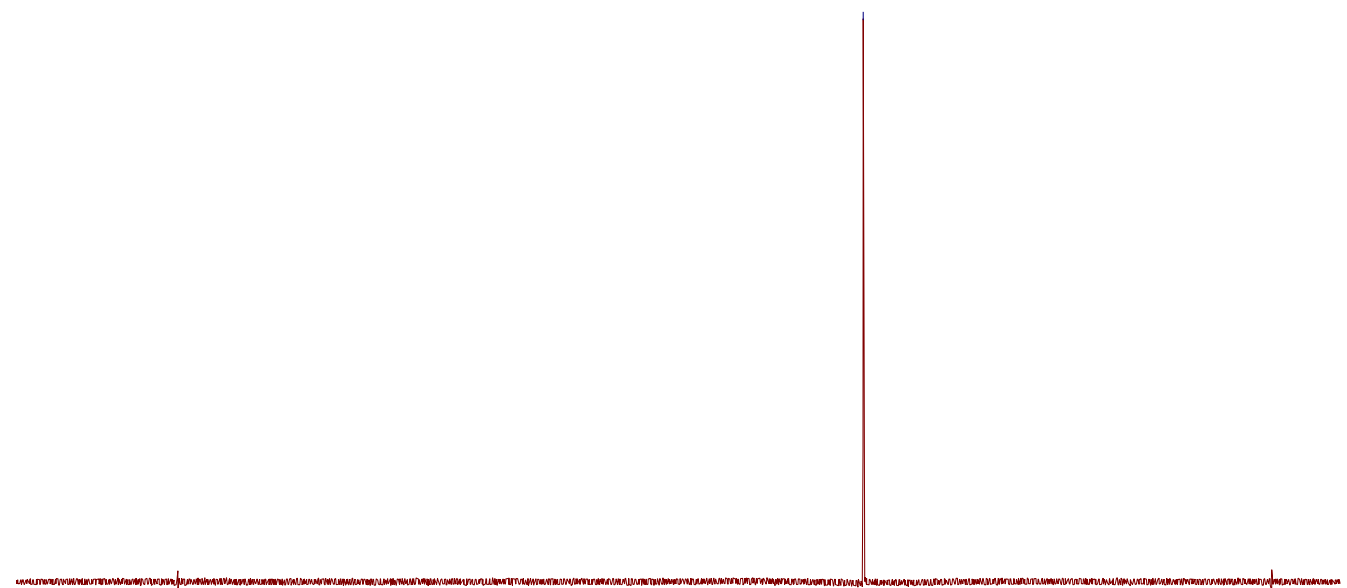

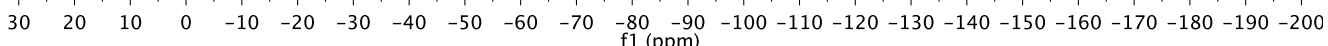


${ }^{1} \mathrm{H}-\mathrm{NMR},{ }^{13} \mathrm{C}-\mathrm{NMR}$ and ${ }^{19} \mathrm{~F}-\mathrm{NMR}$ Spectra for compound $\mathbf{2 6}$

$\stackrel{\mathrm{O}}{\mathrm{Q}}$

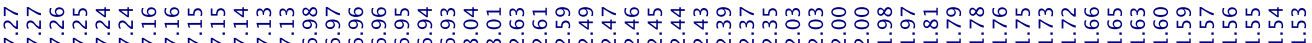

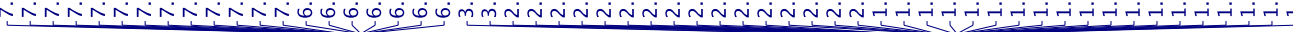
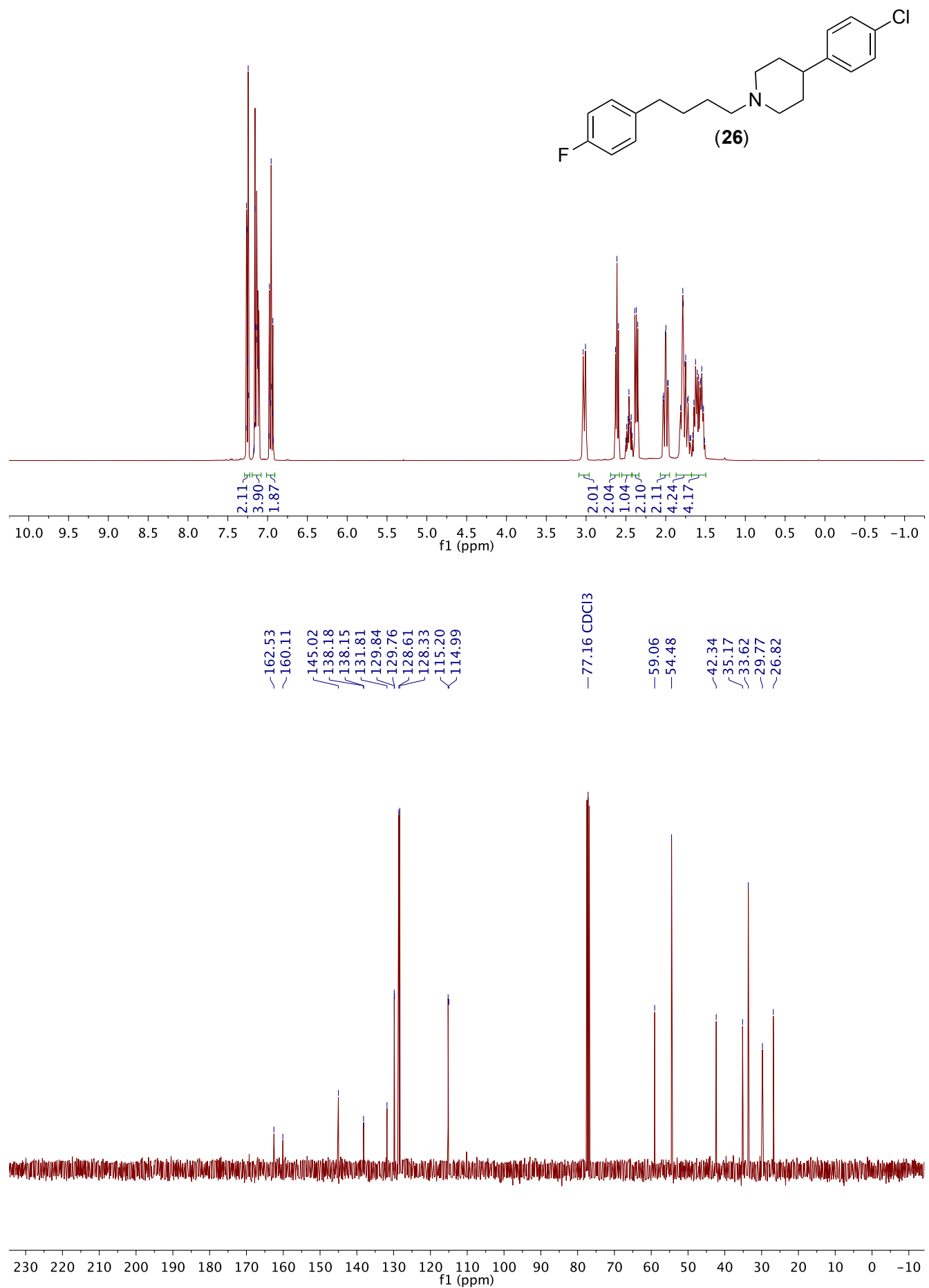


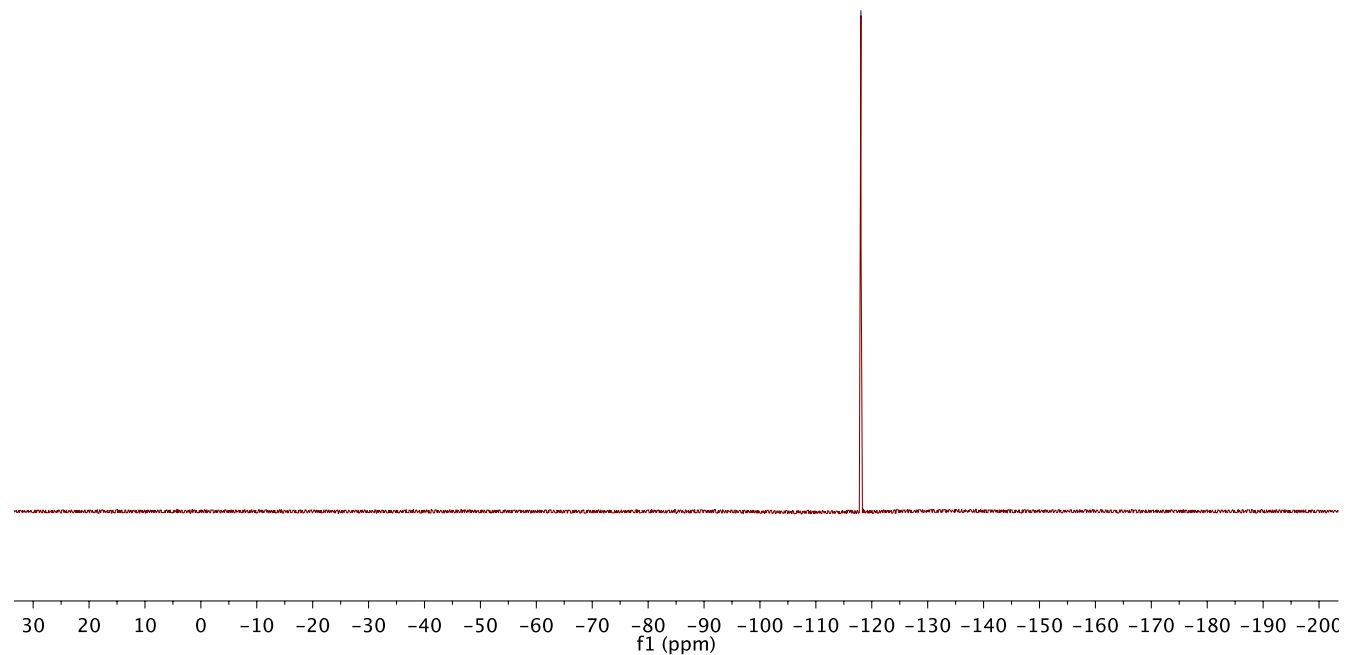

S-52 


\section{${ }^{1}$ H-NMR and ${ }^{13}$ C-NMR Spectra for compound 27}

$\stackrel{m}{\mathrm{U}}$

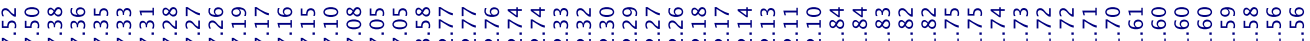

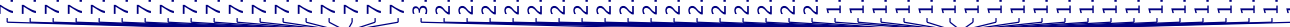

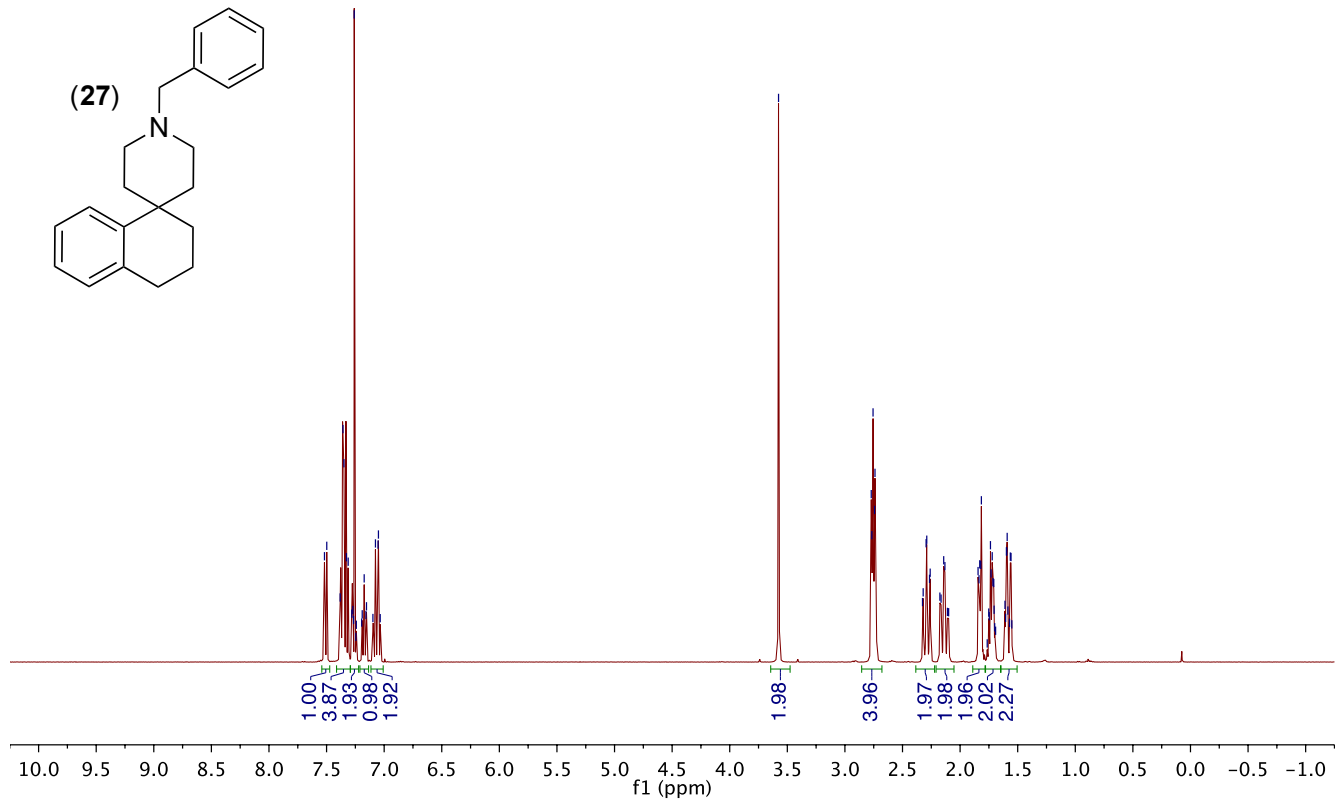

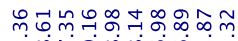
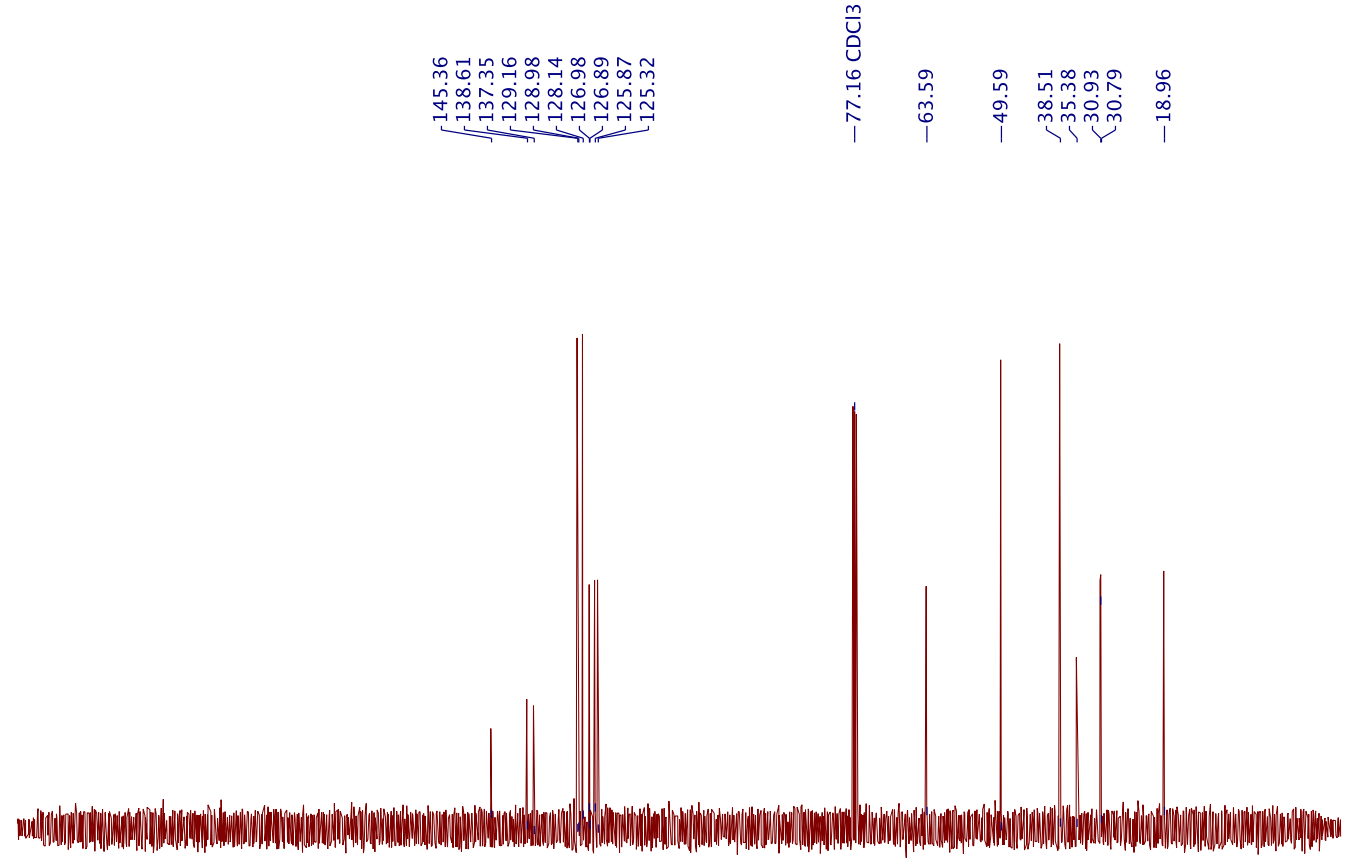

$\begin{array}{lllllllllllllllllllllllll}230 & 220 & 210 & 200 & 190 & 180 & 170 & 160 & 150 & 140 & 130 & 120 & 110 & 100 & 90 & 80 & 70 & 60 & 50 & 40 & 30 & 20 & 10 & 0 & -10\end{array}$ 


\section{${ }^{1} \mathrm{H}-\mathrm{NMR}$ and ${ }^{13} \mathrm{C}-\mathrm{NMR}$ Spectra for compound $\mathbf{1 a}$}
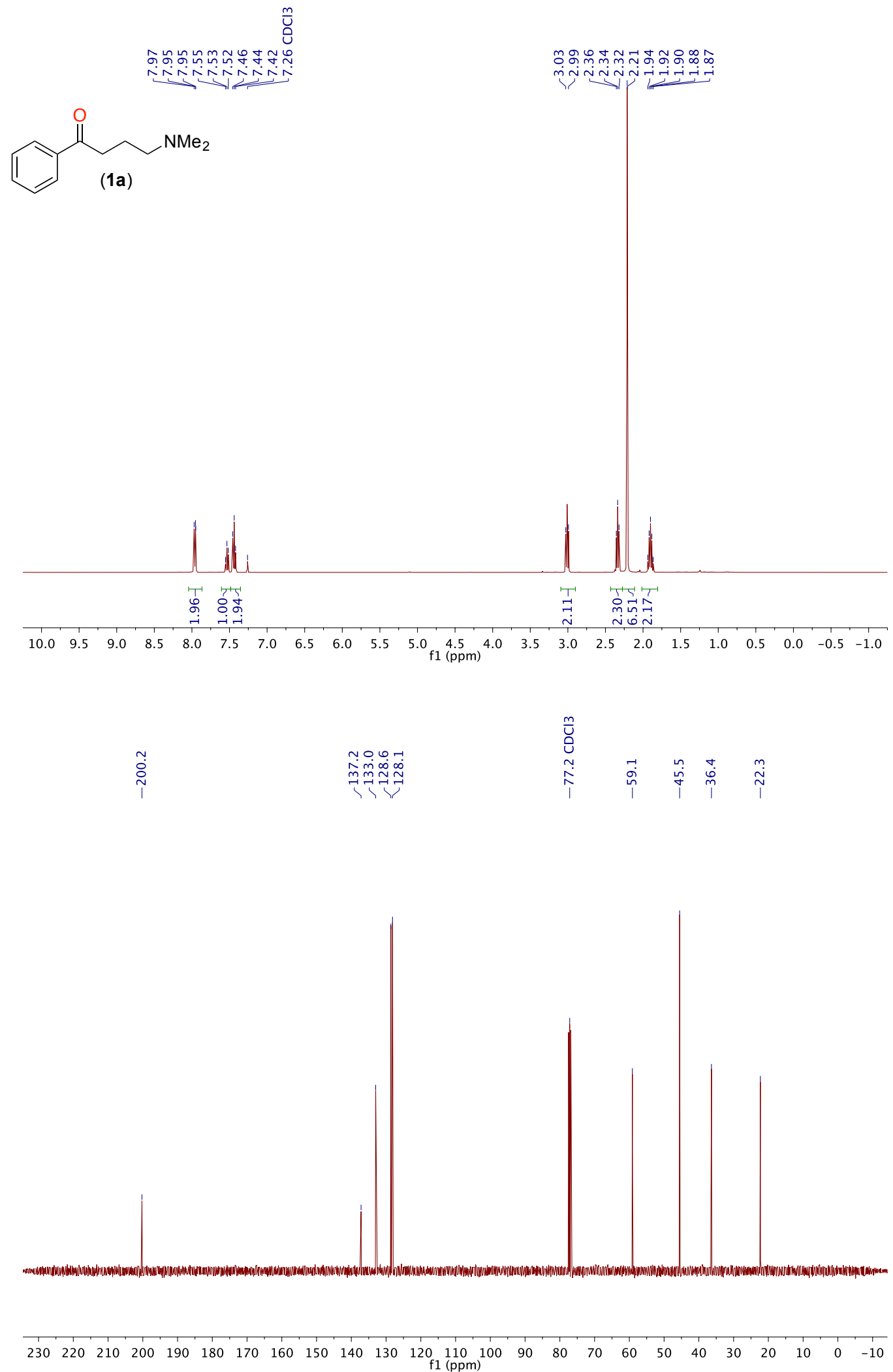
${ }^{1} \mathrm{H}-\mathrm{NMR}$ and ${ }^{13} \mathrm{C}-\mathrm{NMR}$ Spectra for compound 2a
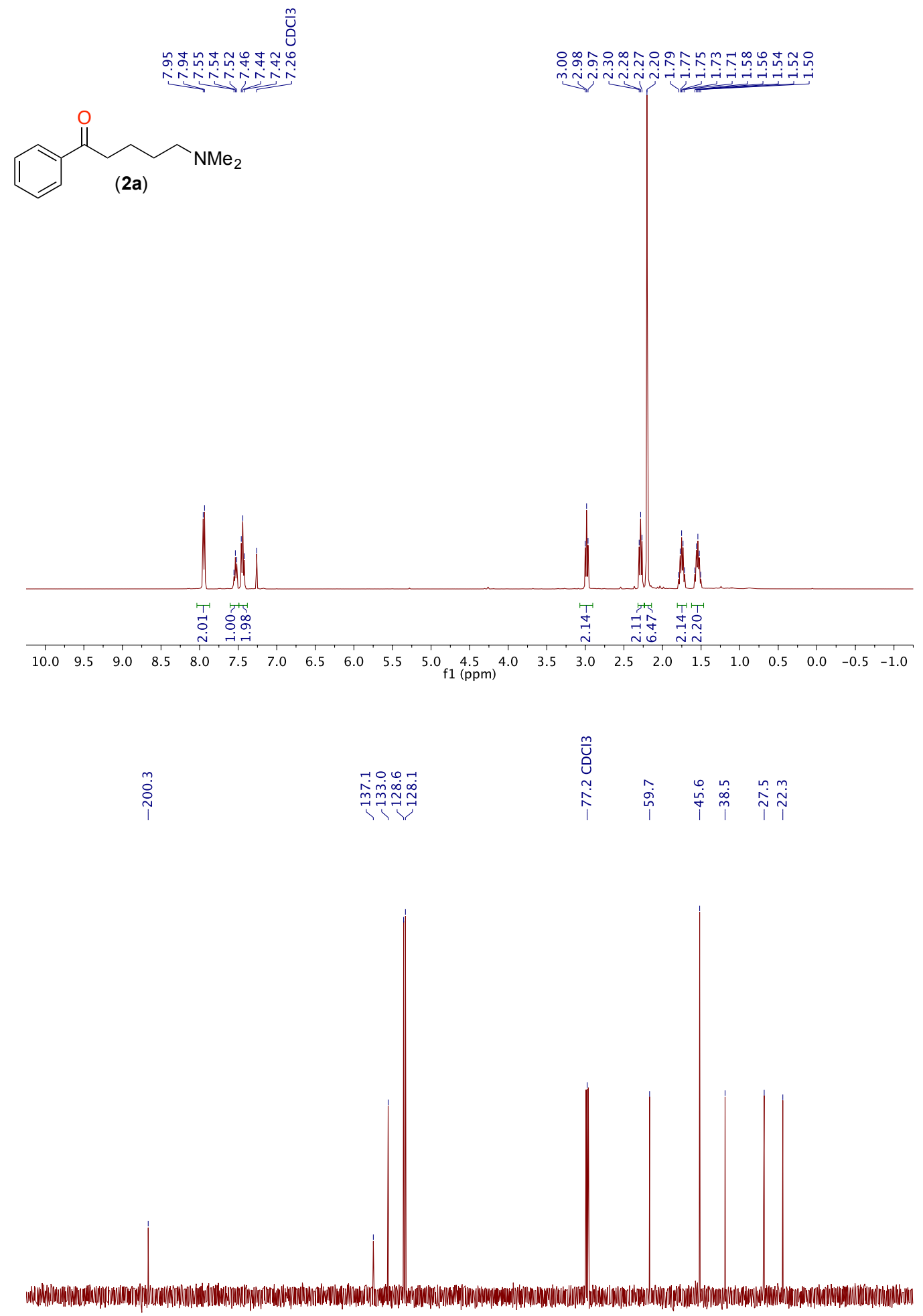

$\begin{array}{llllllllllllllllllllllllllll}230 & 220 & 210 & 200 & 190 & 180 & 170 & 160 & 150 & 140 & 130 & 120 & \mathrm{f} 1 \mathrm{lp}(\mathrm{pm}) & 100 & 90 & 80 & 70 & 60 & 50 & 40 & 30 & 20 & 10 & 0 & -10\end{array}$ 
${ }^{1} \mathrm{H}-\mathrm{NMR}$ and ${ }^{13} \mathrm{C}-\mathrm{NMR}$ Spectra for compound 6a

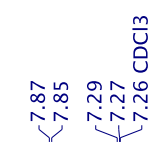

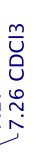

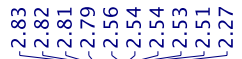

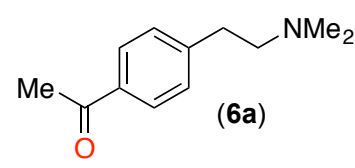
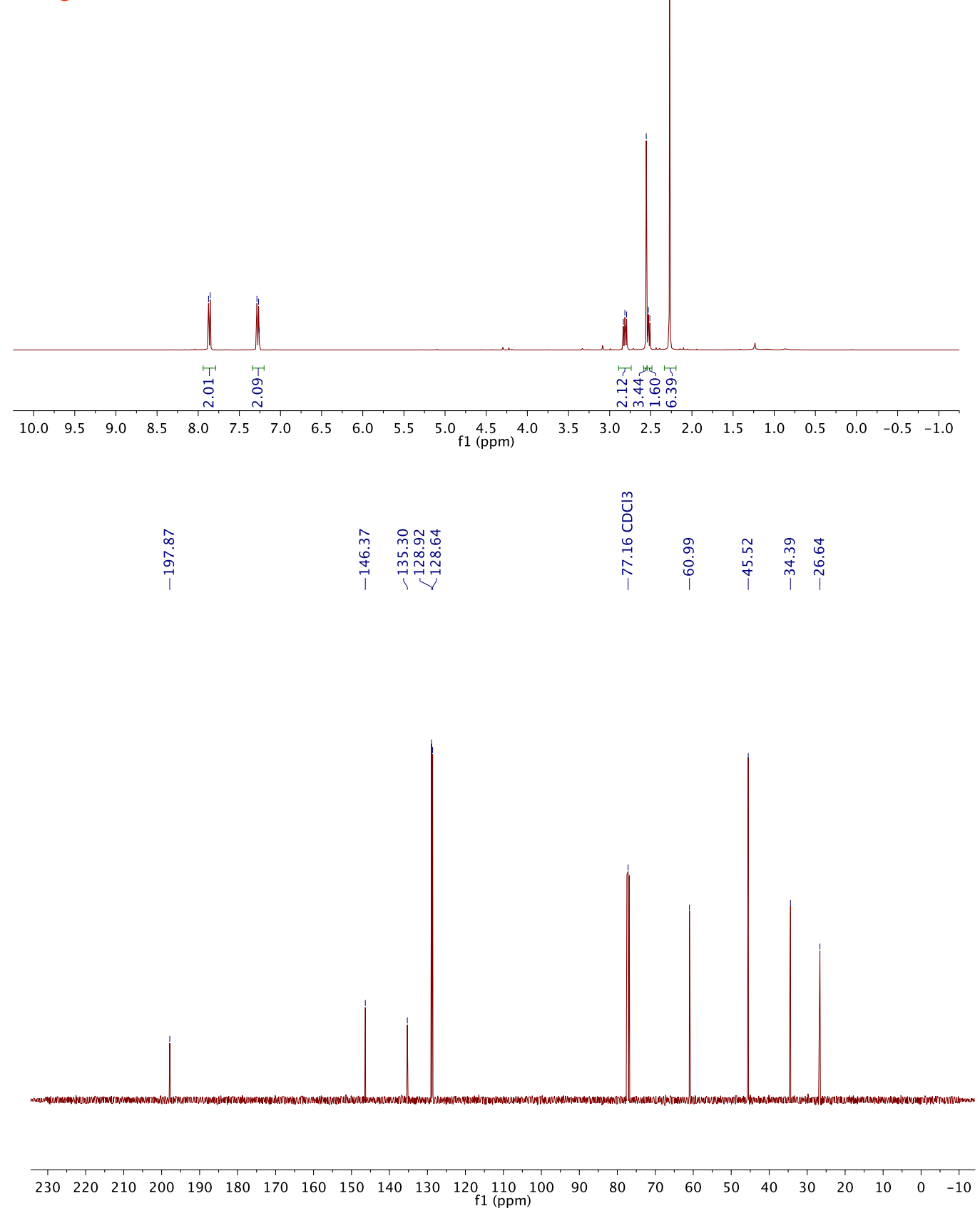
${ }^{1} \mathrm{H}-\mathrm{NMR}$ and ${ }^{13} \mathrm{C}-\mathrm{NMR}$ Spectra for compound $\mathbf{7 a}$
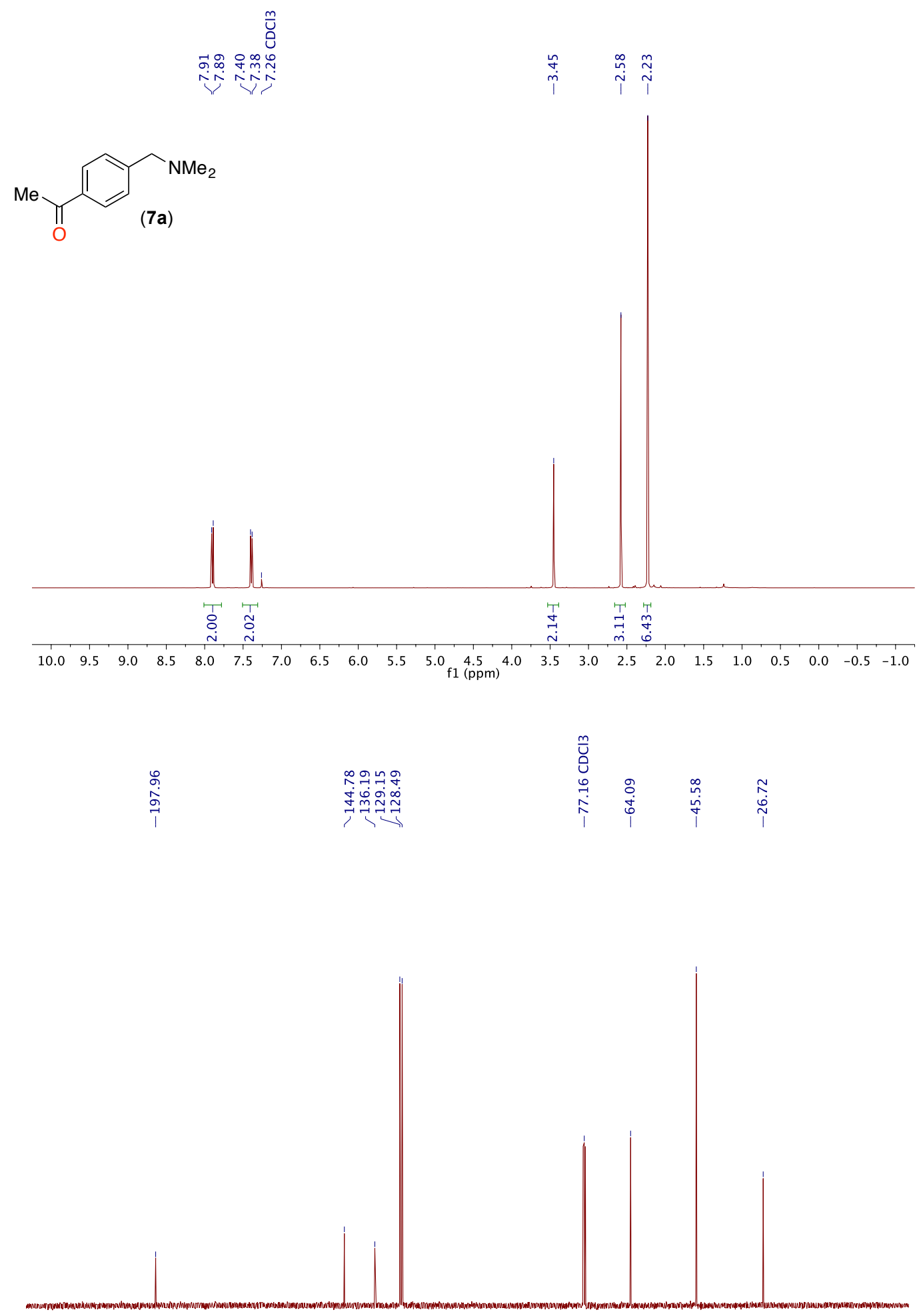

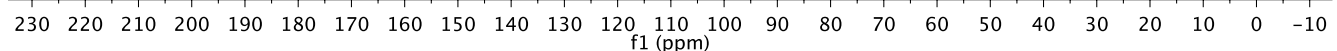


${ }^{1} \mathrm{H}-\mathrm{NMR}$ and ${ }^{13} \mathrm{C}-\mathrm{NMR}$ Spectra for compound 8a
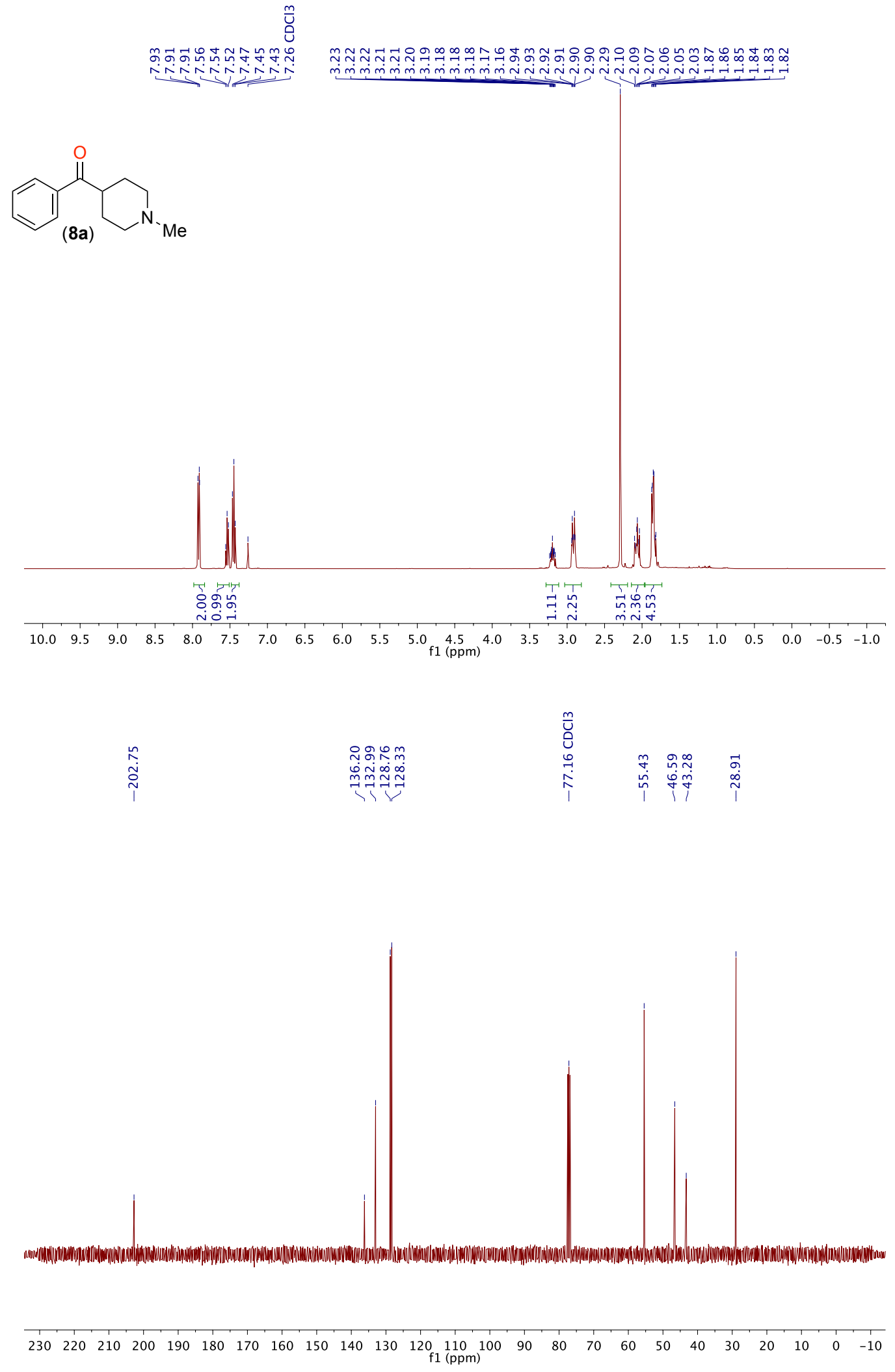
${ }^{1} \mathrm{H}-\mathrm{NMR}$ and ${ }^{13} \mathrm{C}-\mathrm{NMR}$ Spectra for compound 9a
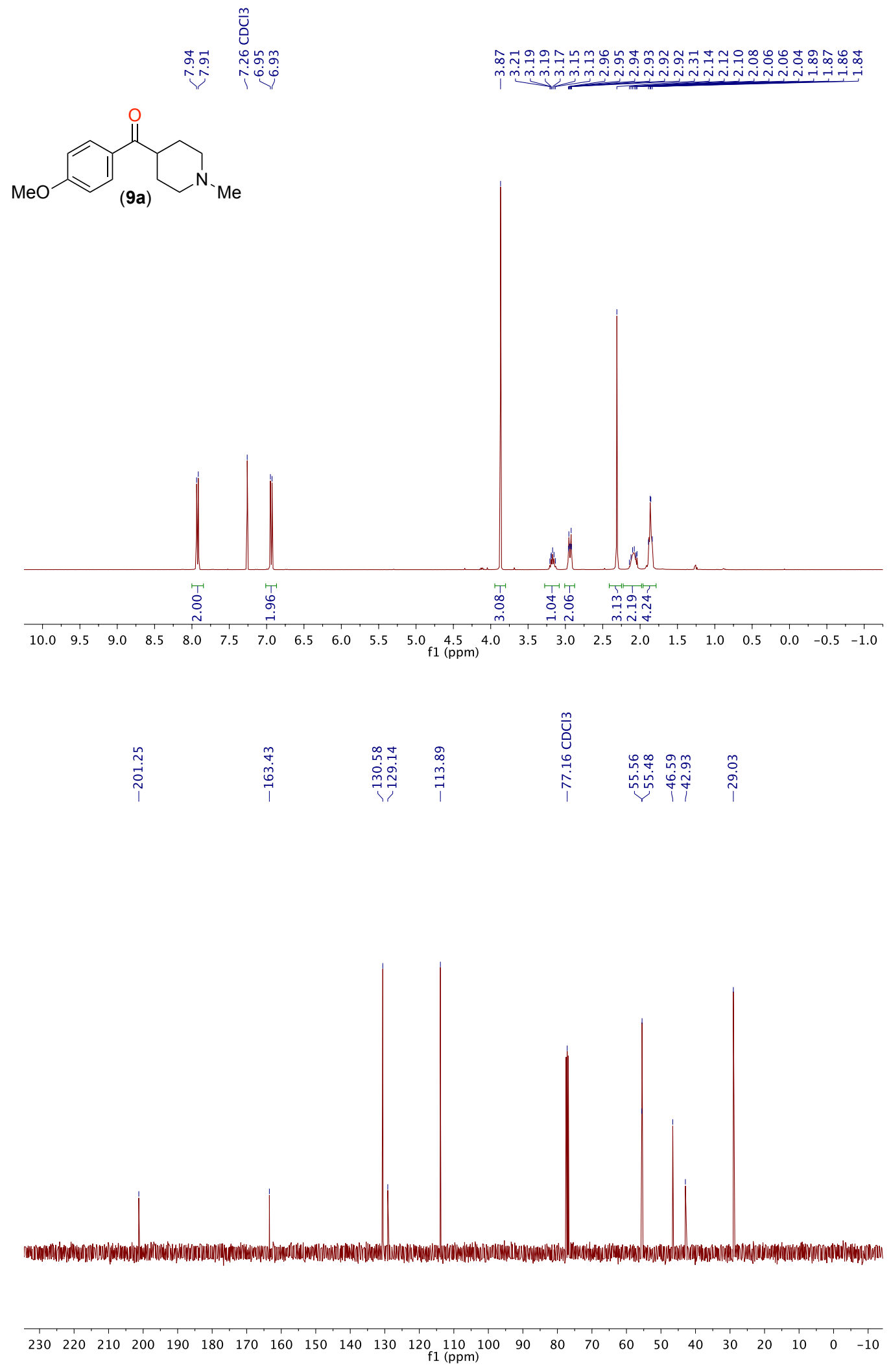
${ }^{1} \mathrm{H}-\mathrm{NMR}$ and ${ }^{13} \mathrm{C}-\mathrm{NMR}$ and ${ }^{19} \mathrm{~F}-\mathrm{NMR}$ Spectra for compound 10a

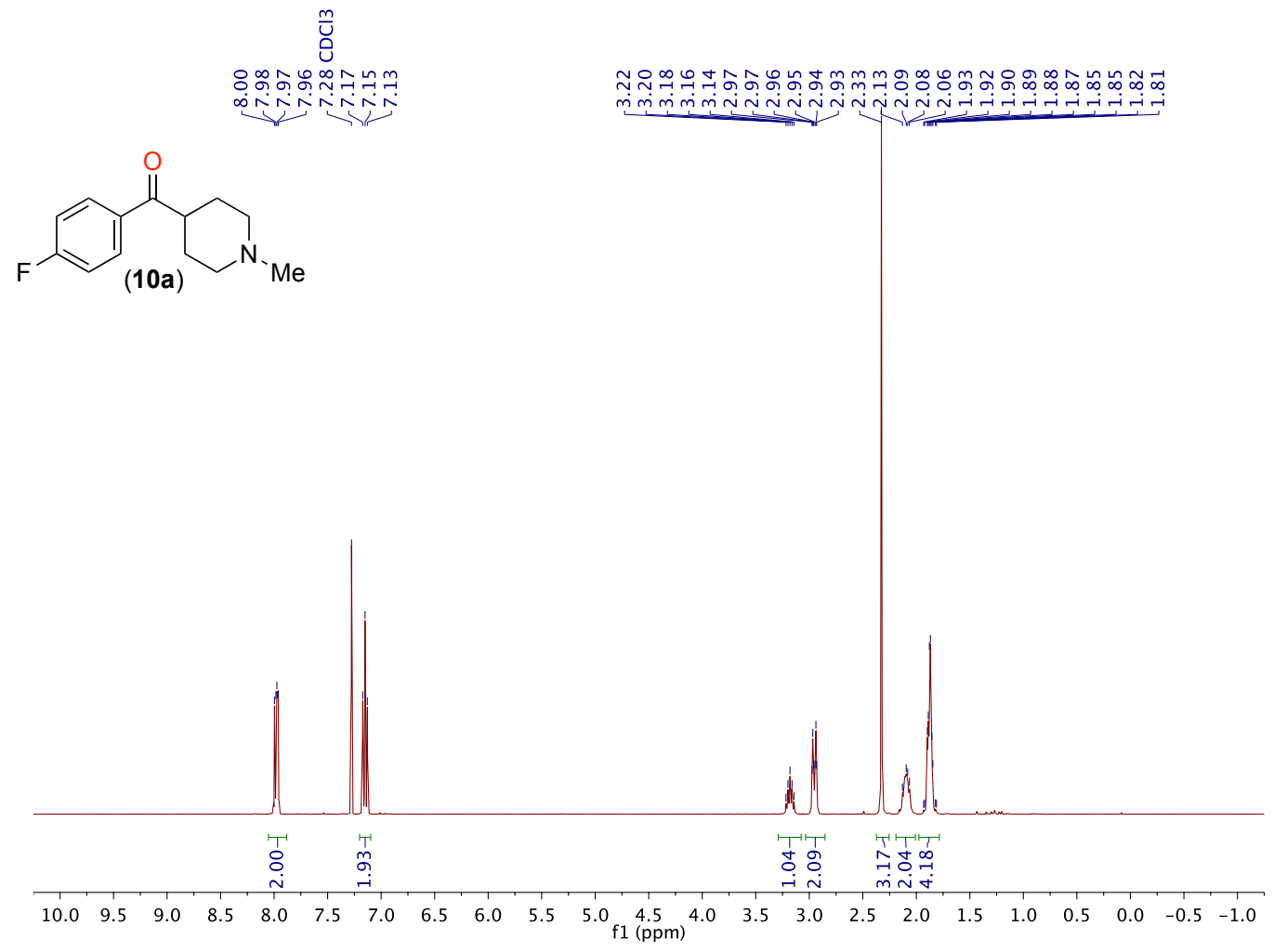

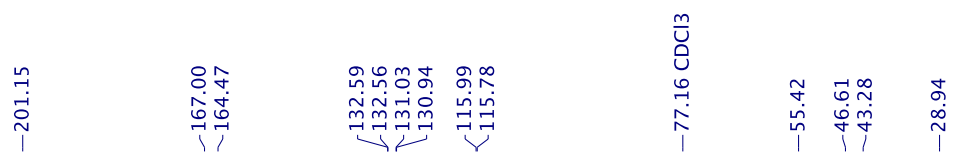

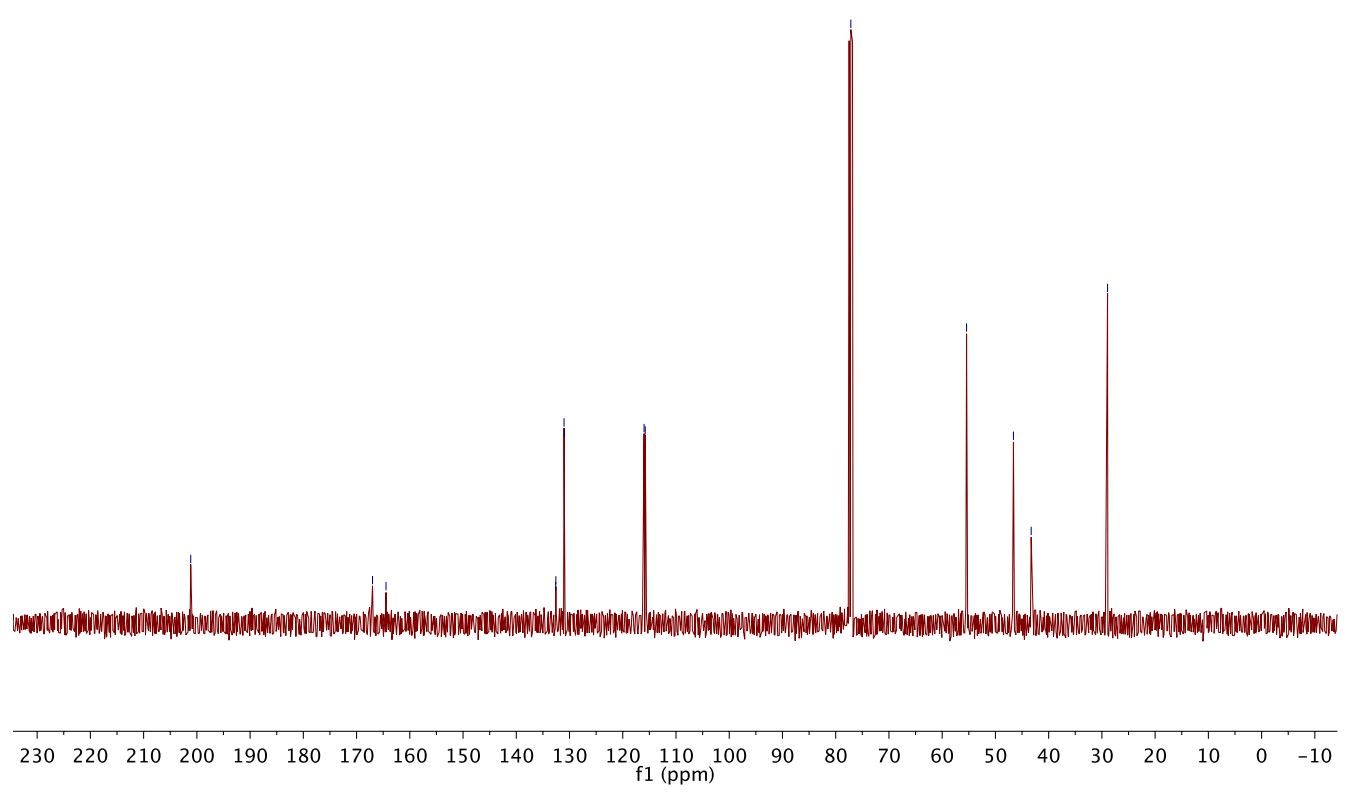




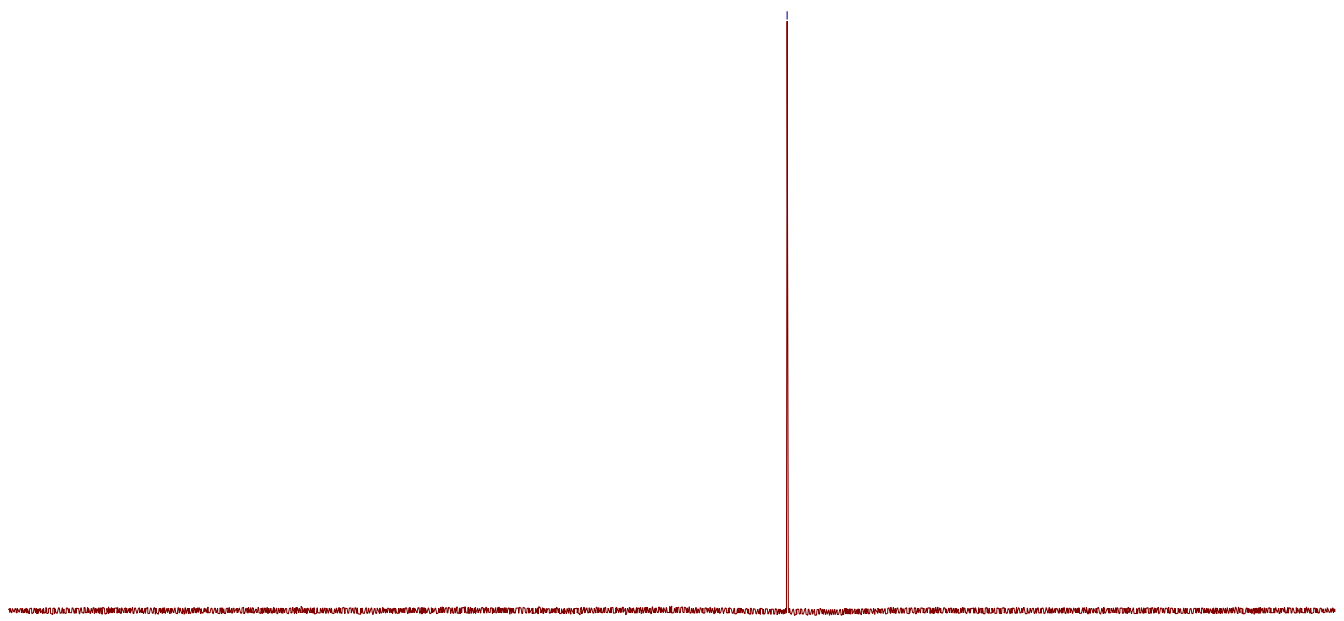

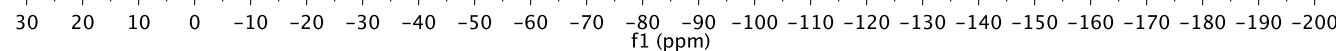


${ }^{1} \mathrm{H}-\mathrm{NMR}$ and ${ }^{13} \mathrm{C}-\mathrm{NMR}$ Spectra for compound $\mathbf{1 1 a}$

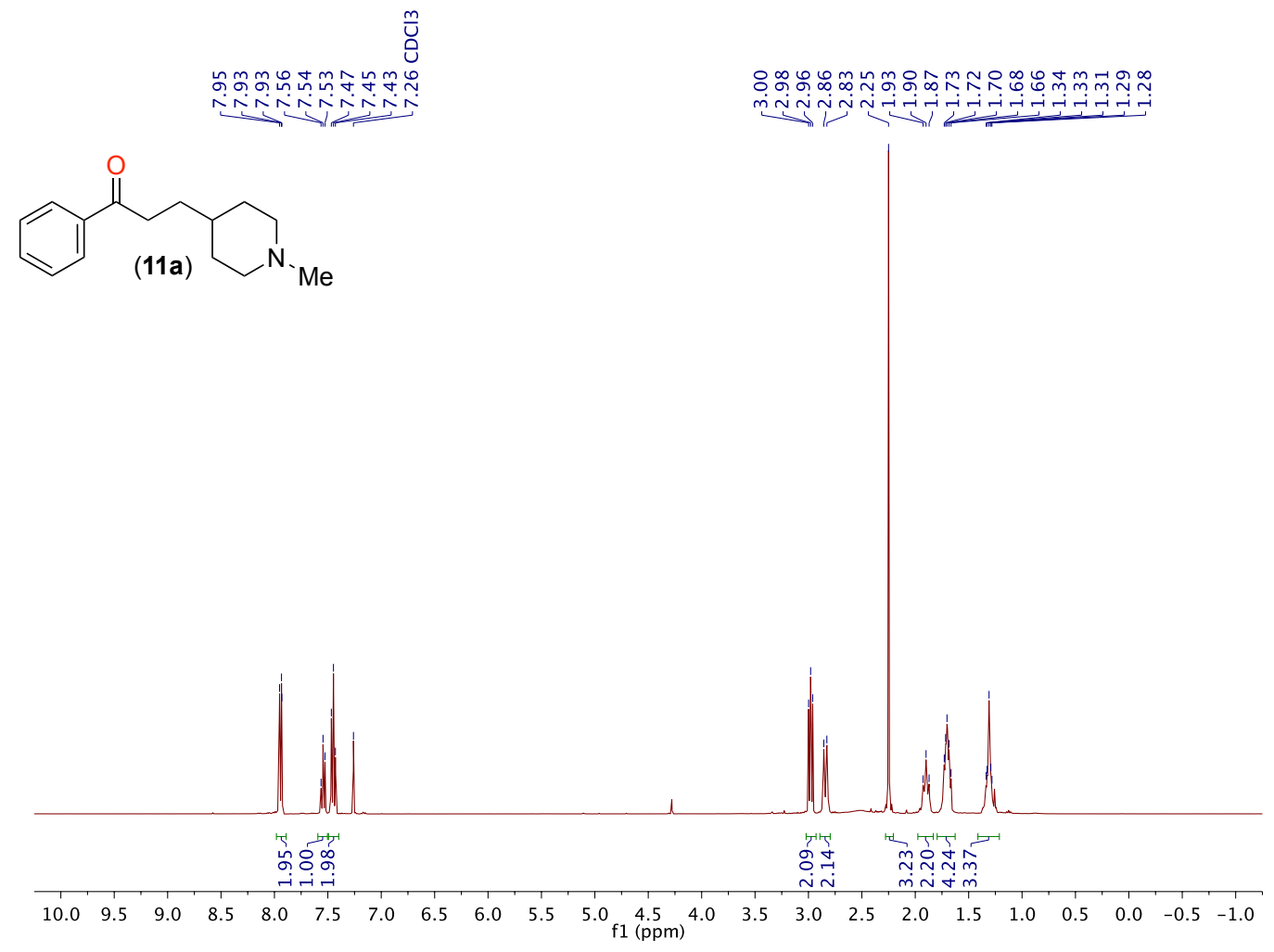

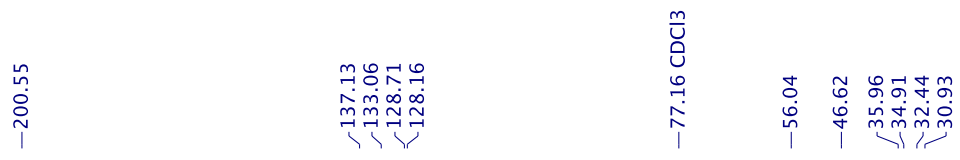

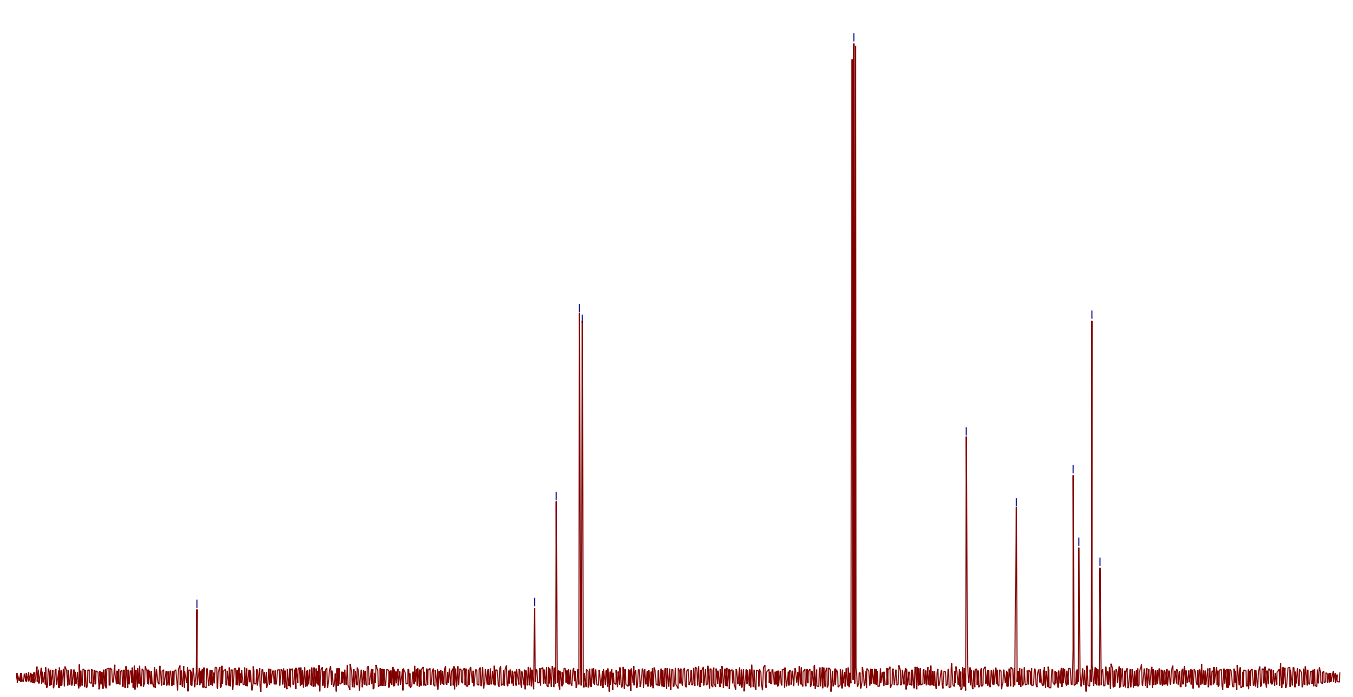

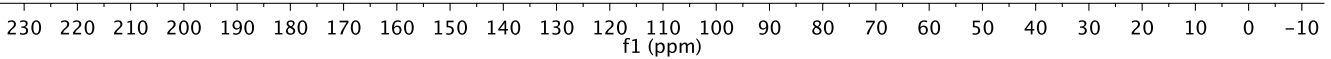


${ }^{1} \mathrm{H}-\mathrm{NMR}$ and ${ }^{13} \mathrm{C}-\mathrm{NMR}$ Spectra for compound 12a
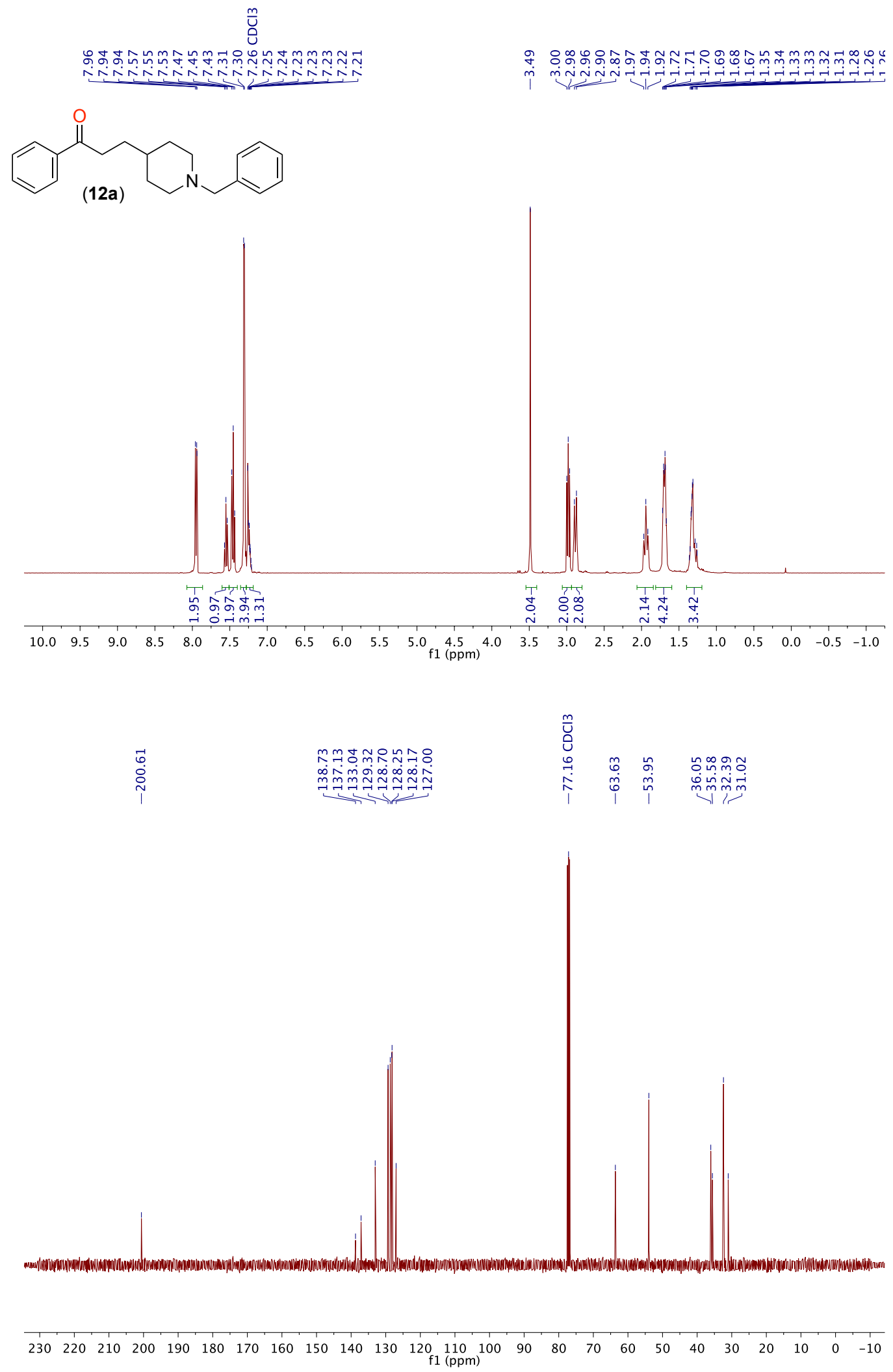
${ }^{1} \mathrm{H}-\mathrm{NMR}$ and ${ }^{13} \mathrm{C}-\mathrm{NMR}$ Spectra for compound 13a
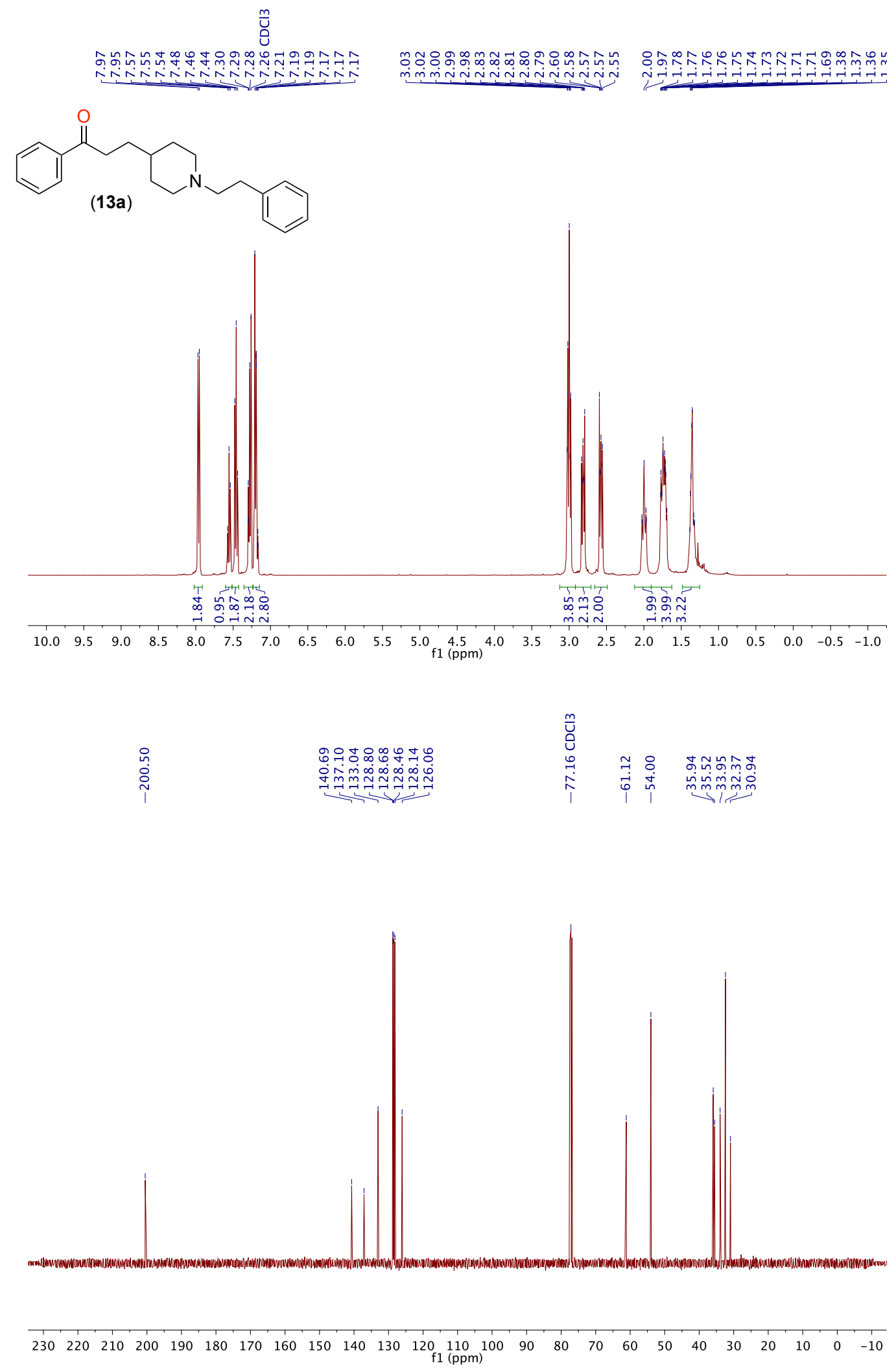

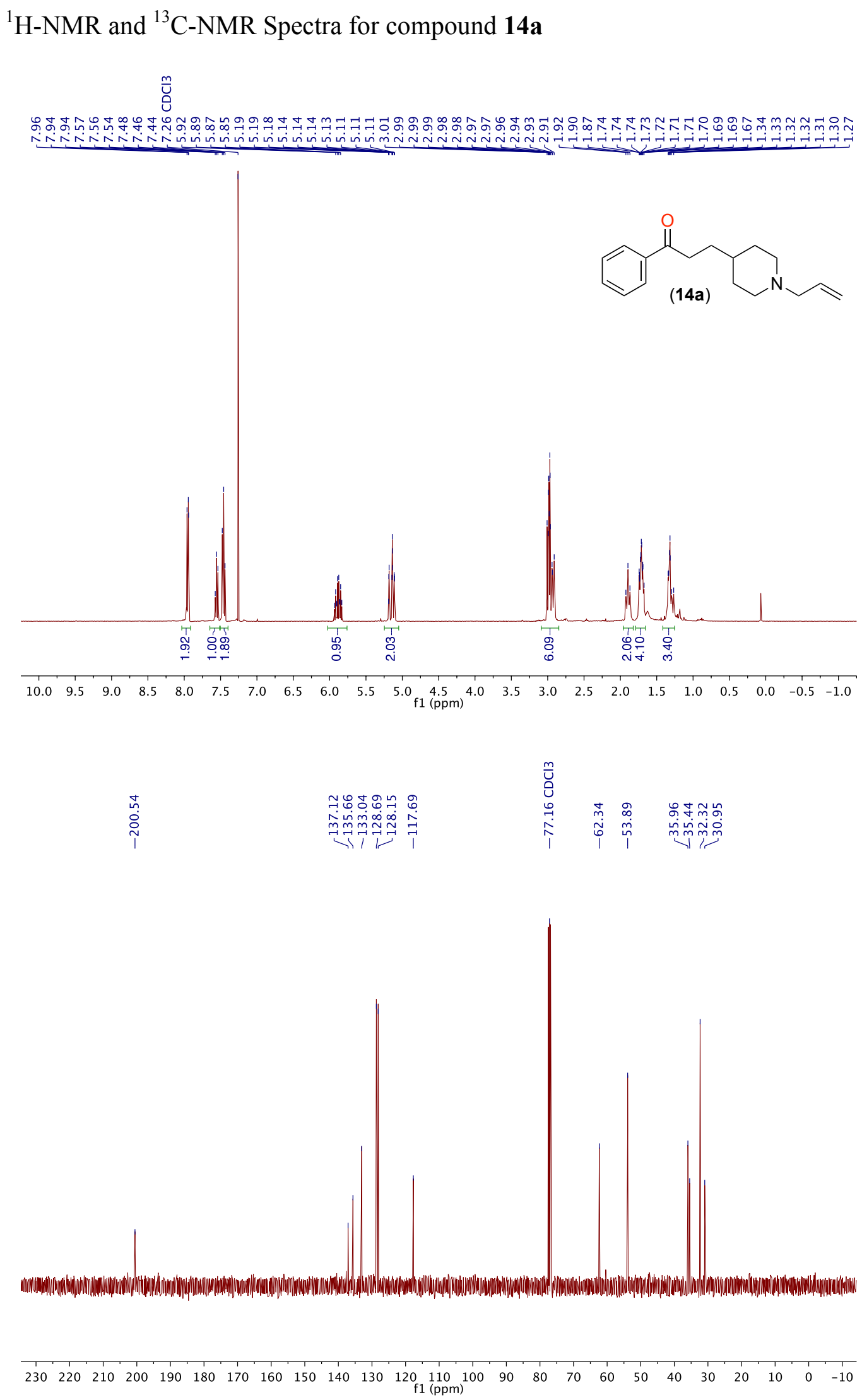
${ }^{1} \mathrm{H}-\mathrm{NMR}$ and ${ }^{13} \mathrm{C}-\mathrm{NMR}$ Spectra for compound 15a
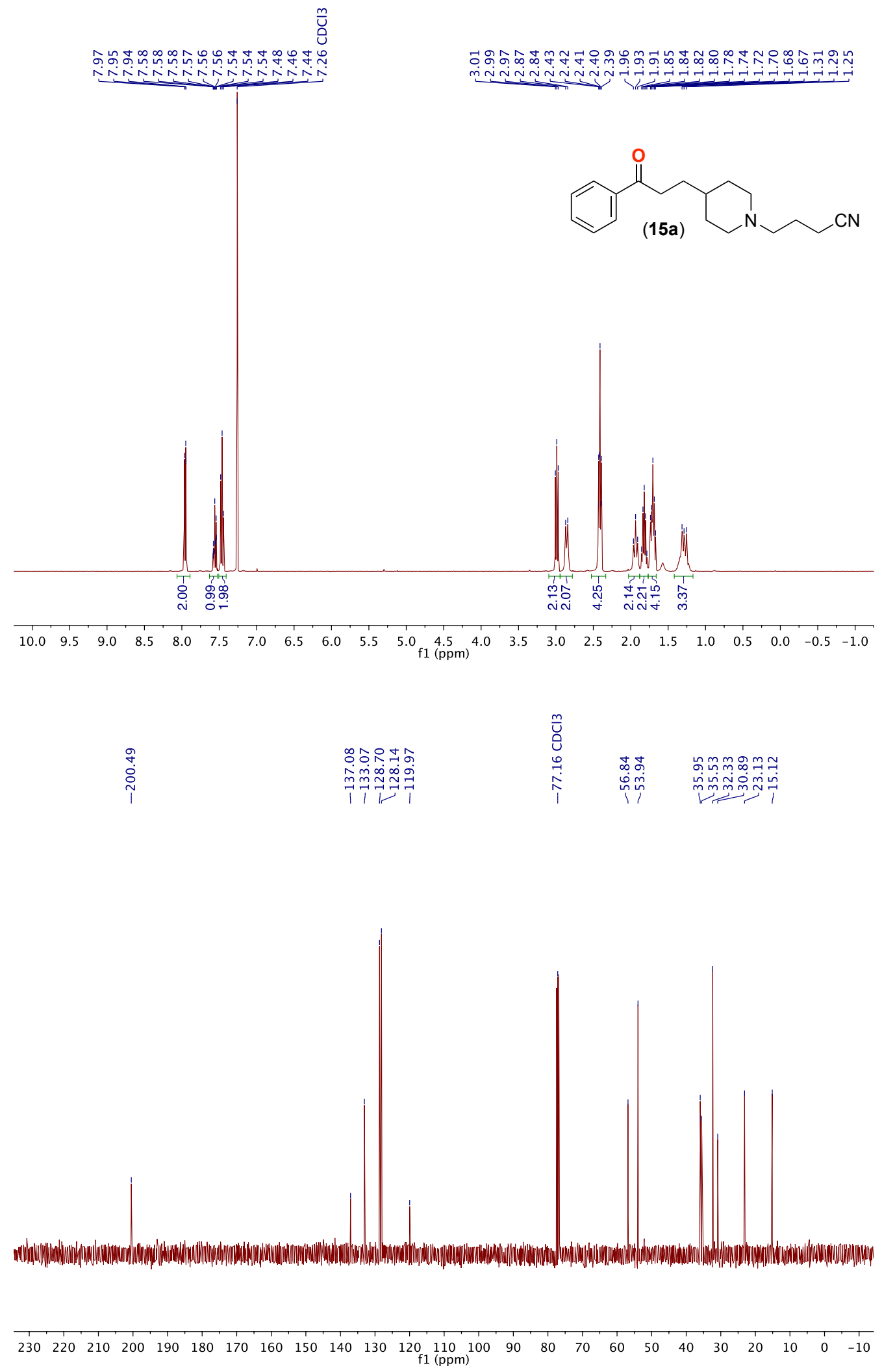
${ }^{1} \mathrm{H}-\mathrm{NMR}$ and ${ }^{13} \mathrm{C}-\mathrm{NMR}$ Spectra for compound 16a
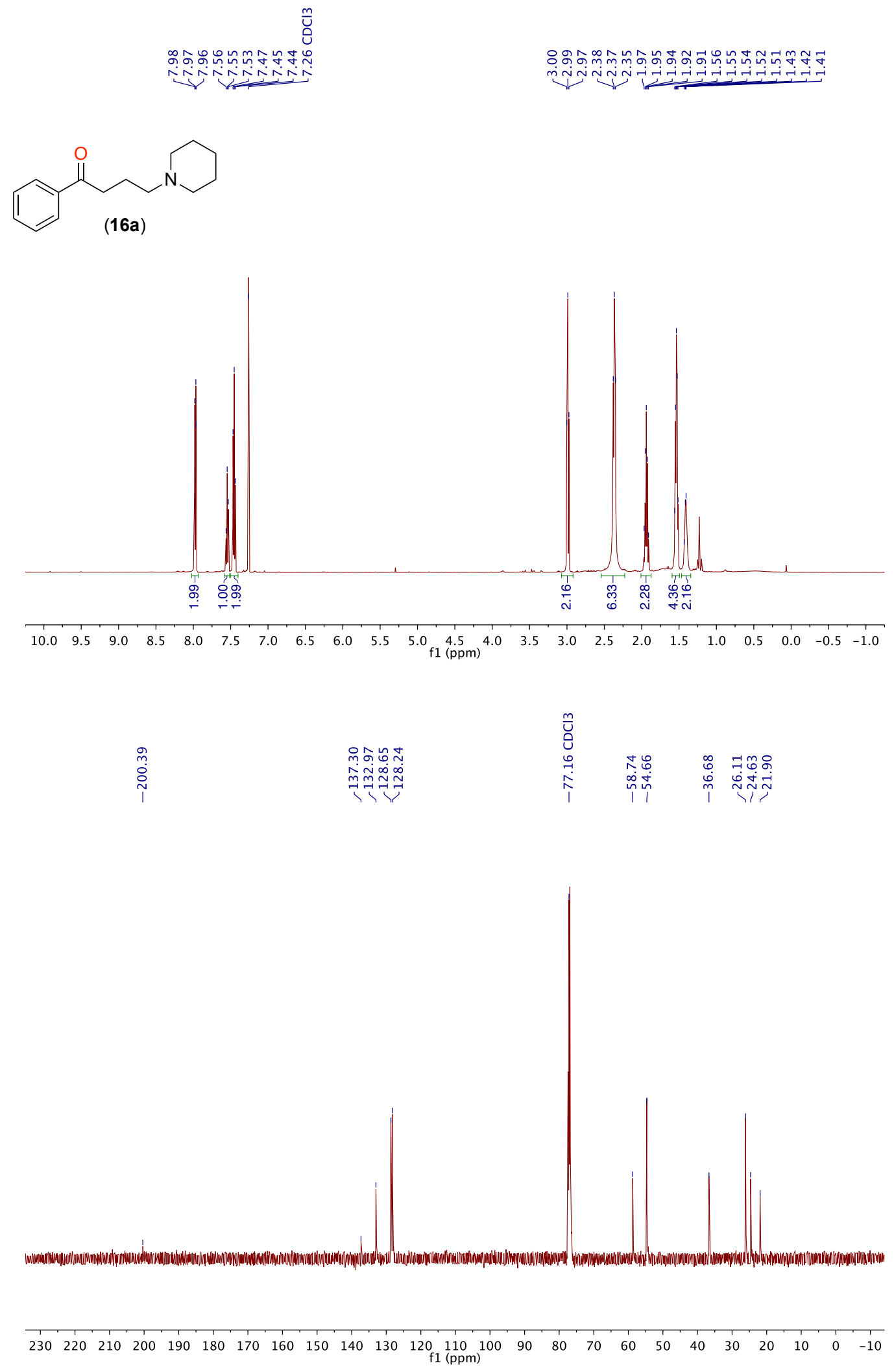
${ }^{1} \mathrm{H}-\mathrm{NMR}$ and ${ }^{13} \mathrm{C}-\mathrm{NMR}$ Spectra for compound 17a

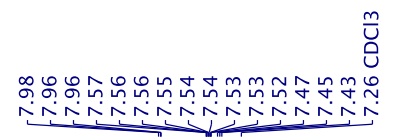

$\overbrace{(17 a)}^{O} N$

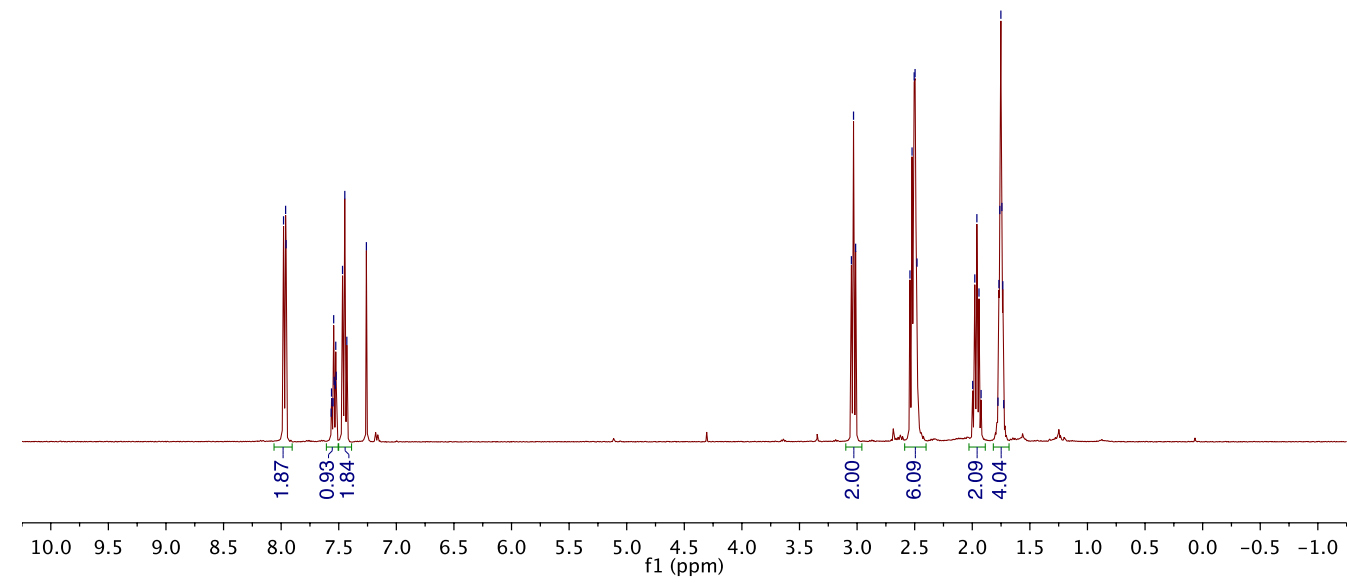

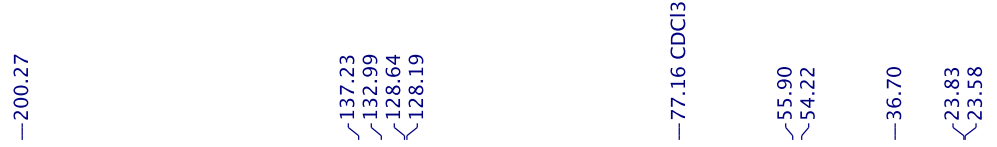

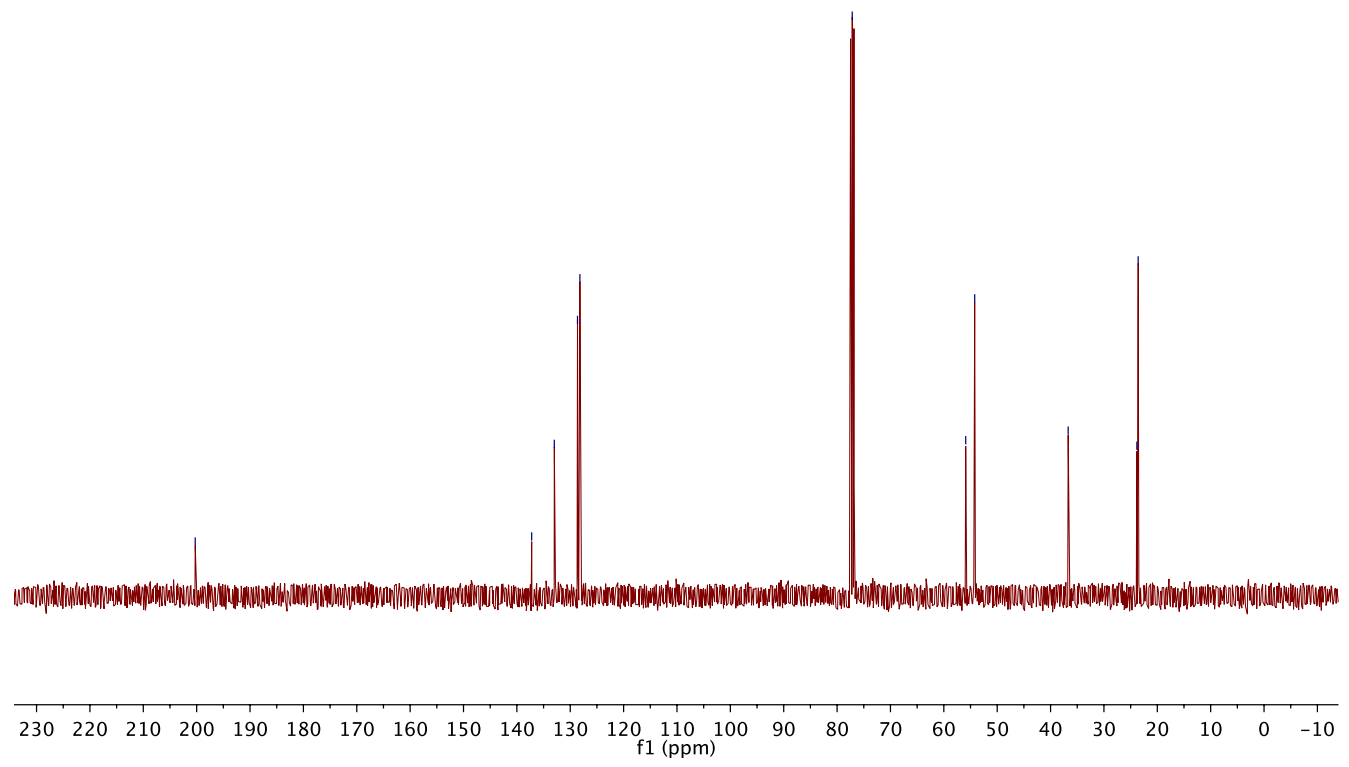


${ }^{1} \mathrm{H}-\mathrm{NMR}$ and ${ }^{13} \mathrm{C}-\mathrm{NMR}$ Spectra for compound 18a

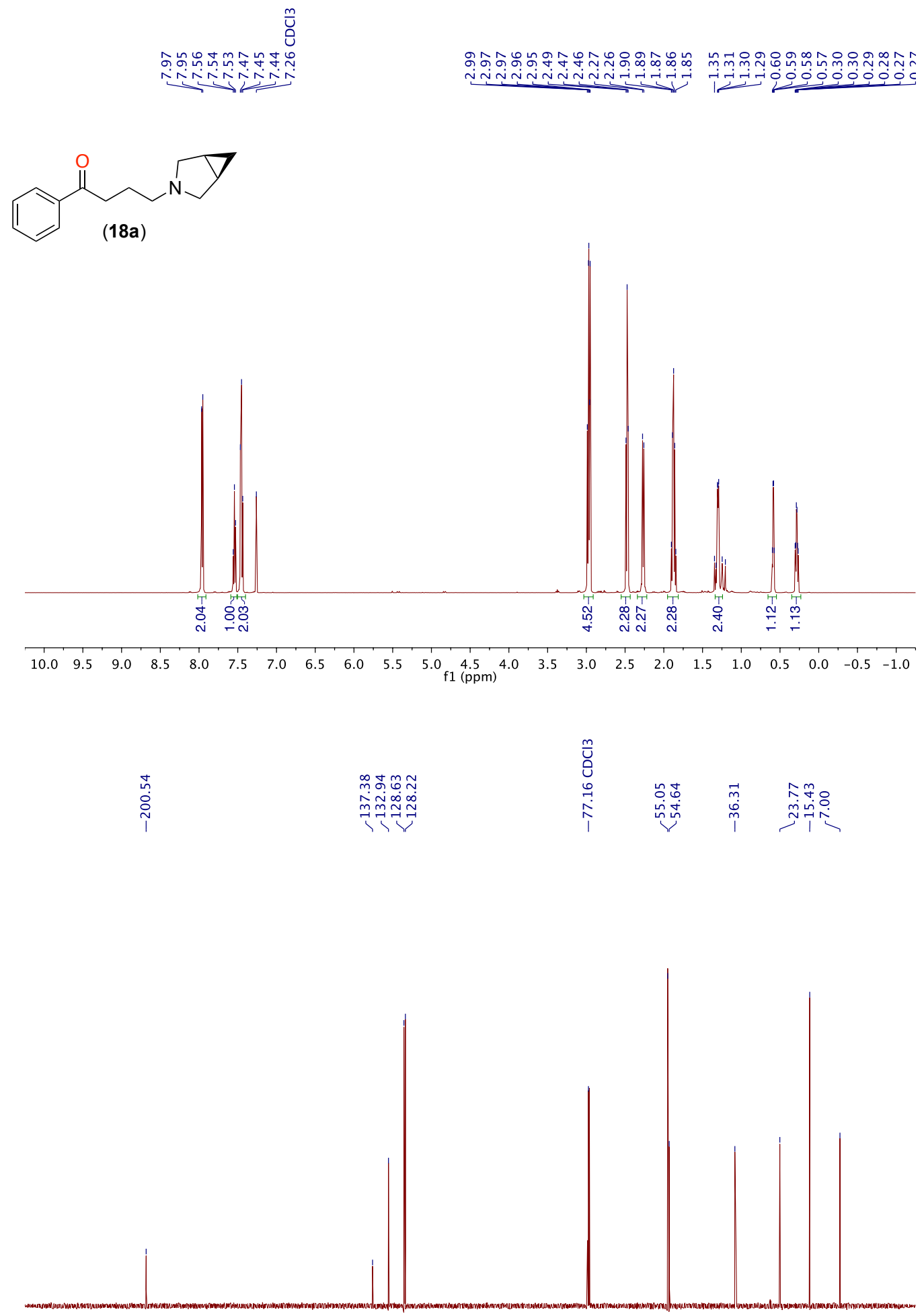

$\begin{array}{lllllllllllllllllllllllllll}230 & 220 & 210 & 200 & 190 & 180 & 170 & 160 & 150 & 140 & 130 & 120 & 110 & 100 & 90 & 80 & 70 & 60 & 50 & 40 & 30 & 20 & 10 & 0 & -10\end{array}$ 
${ }^{1} \mathrm{H}-\mathrm{NMR}$ and ${ }^{13} \mathrm{C}-\mathrm{NMR}$ Spectra for compound 19a
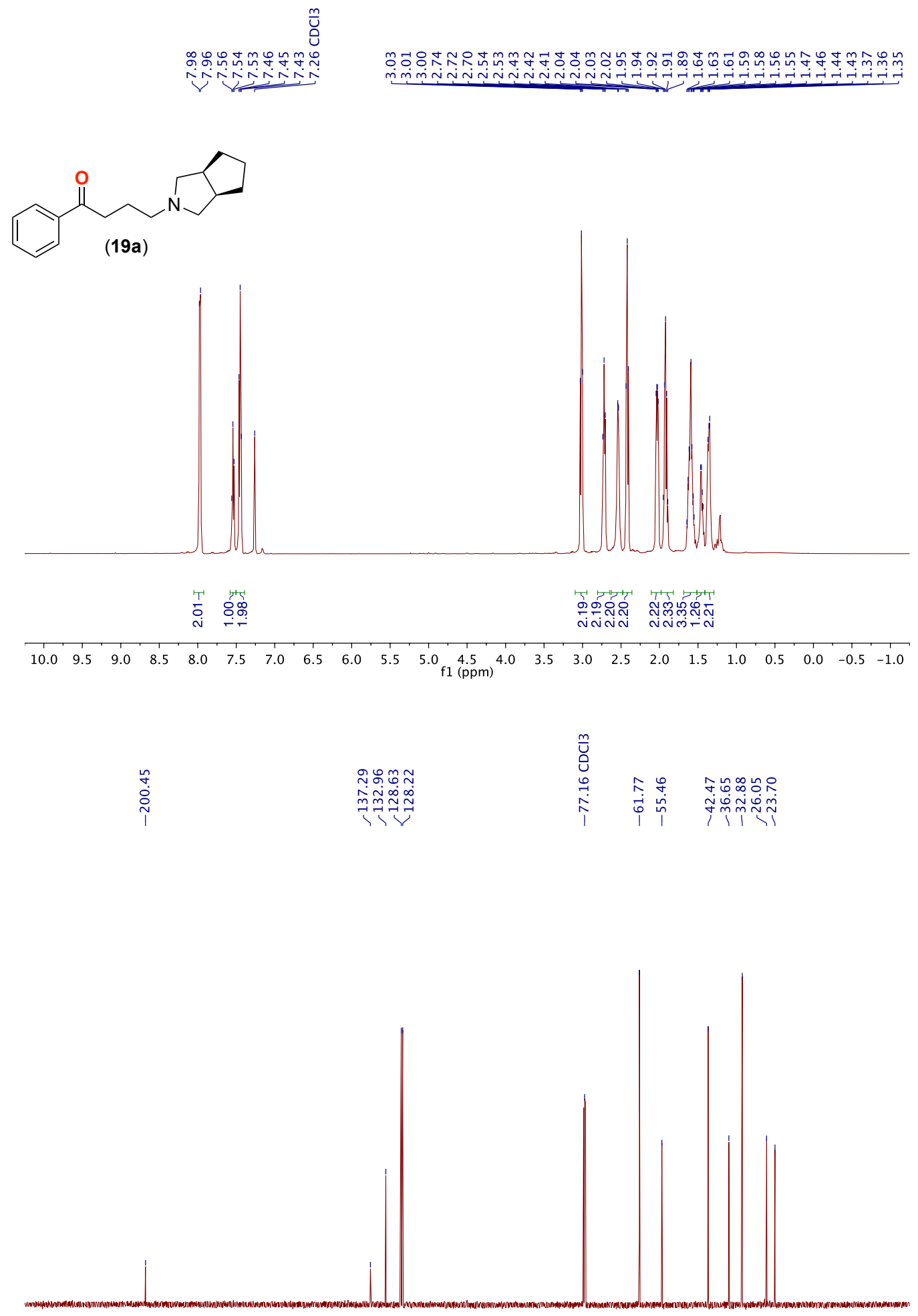

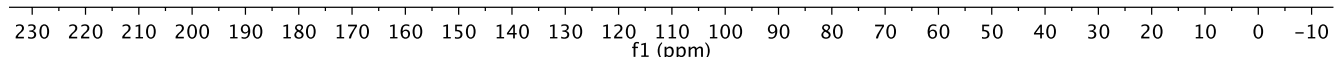


${ }^{1} \mathrm{H}-\mathrm{NMR}$ and ${ }^{13} \mathrm{C}-\mathrm{NMR}$ Spectra for compound 20a

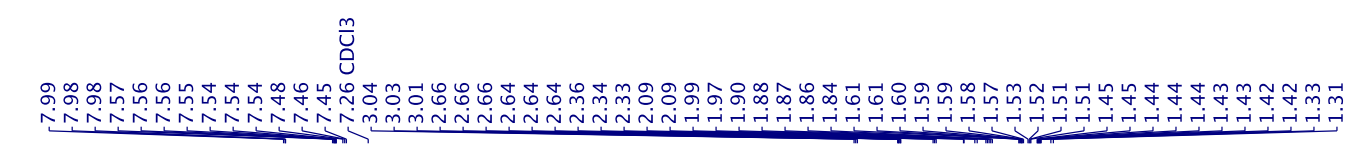

$\underbrace{O}_{(20 a)}$
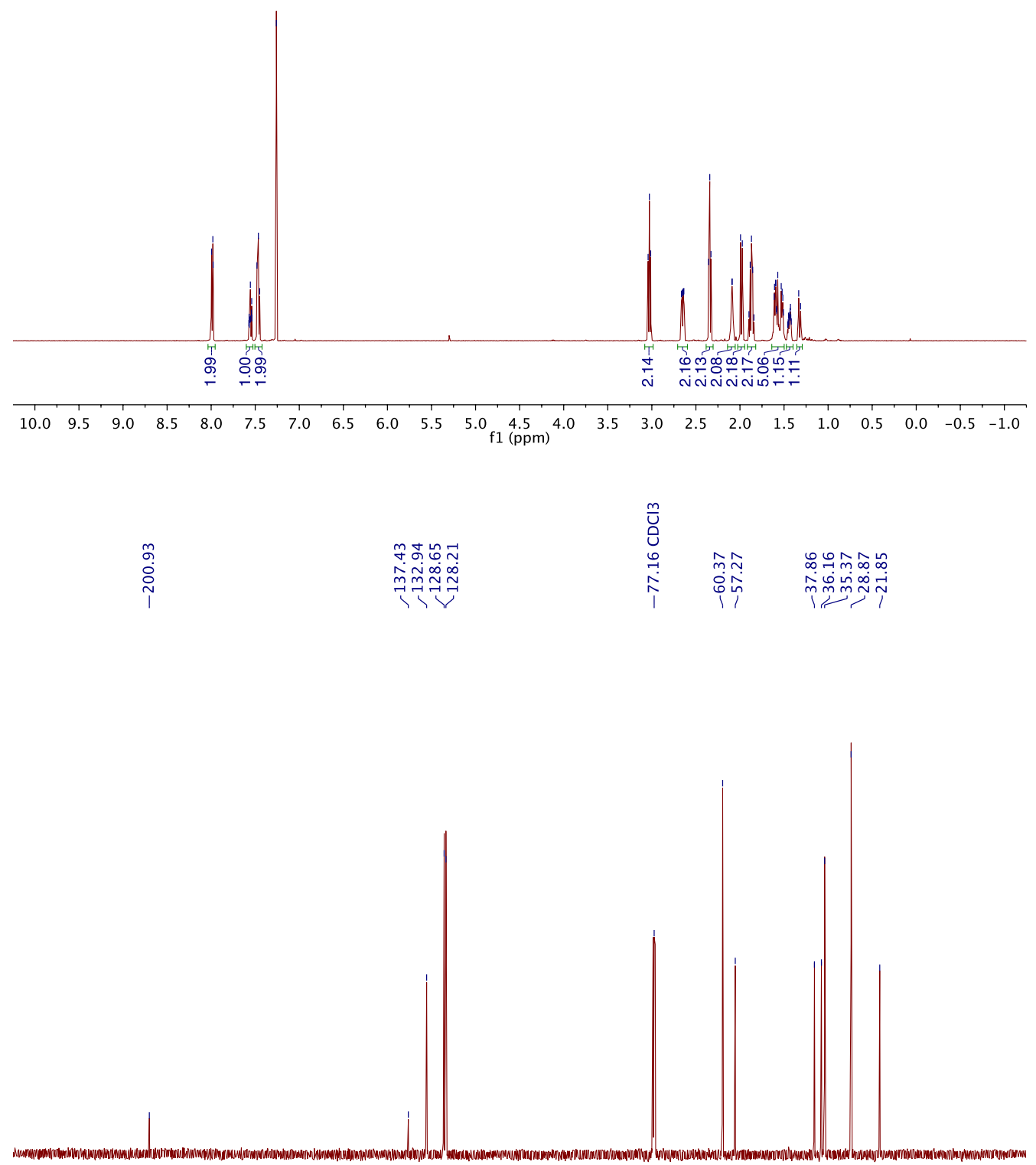

$\begin{array}{lllllllllllllllllllllllllllllll}230 & 220 & 210 & 200 & 190 & 180 & 170 & 160 & 150 & 140 & 130 & 120 & 110 & 100 & 90 & 80 & 70 & 60 & 50 & 40 & 30 & 20 & 10 & 0 & -10\end{array}$ 
${ }^{1} \mathrm{H}-\mathrm{NMR}$ and ${ }^{13} \mathrm{C}-\mathrm{NMR}$ Spectra for compound 21a

$\stackrel{m}{\breve{~}}$

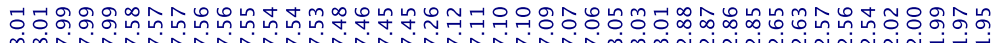

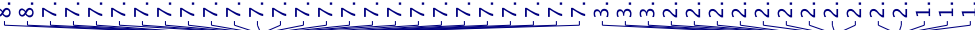
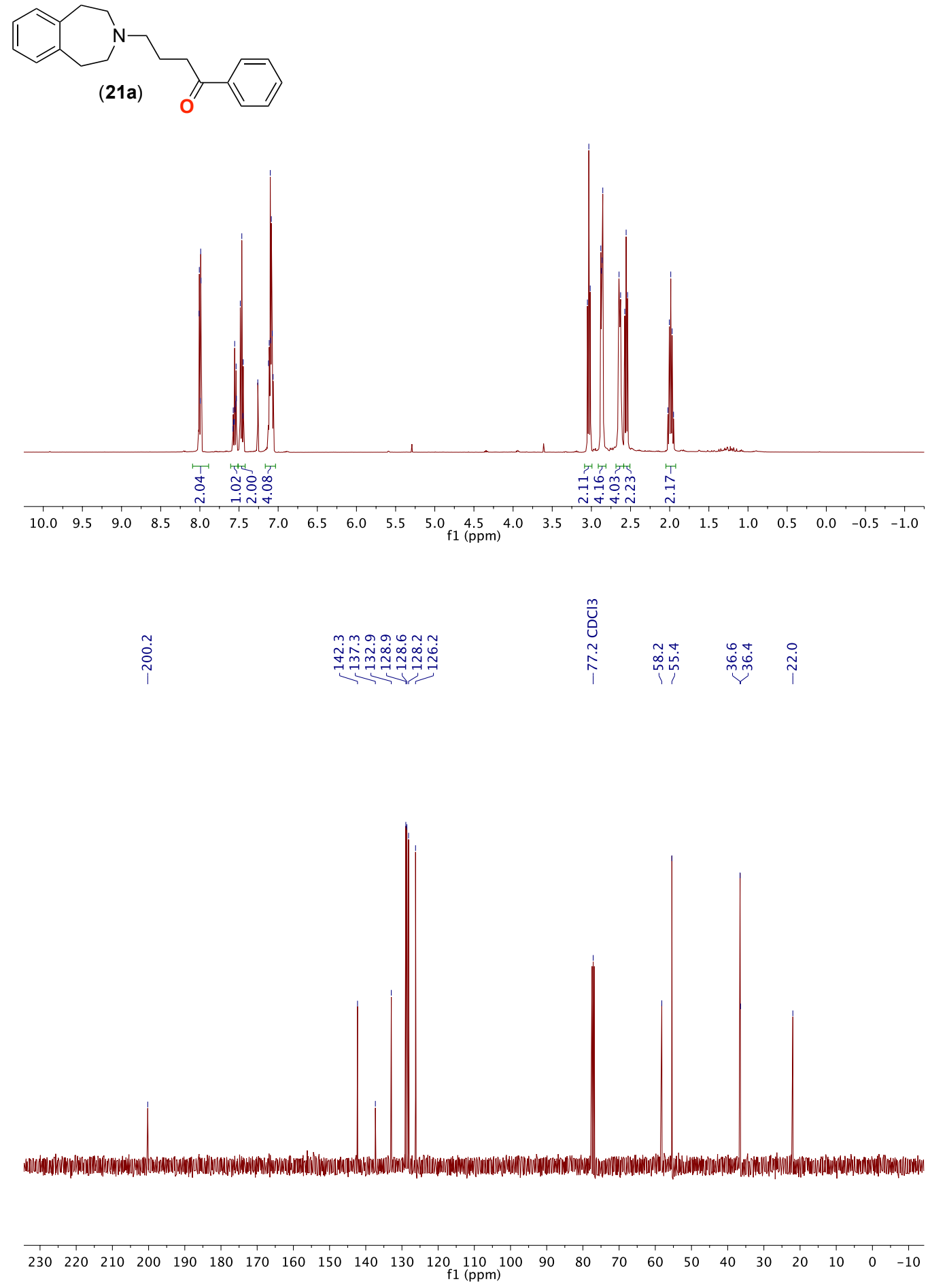
${ }^{1} \mathrm{H}-\mathrm{NMR}$ and ${ }^{13} \mathrm{C}-\mathrm{NMR}$ Spectra for compound 22a
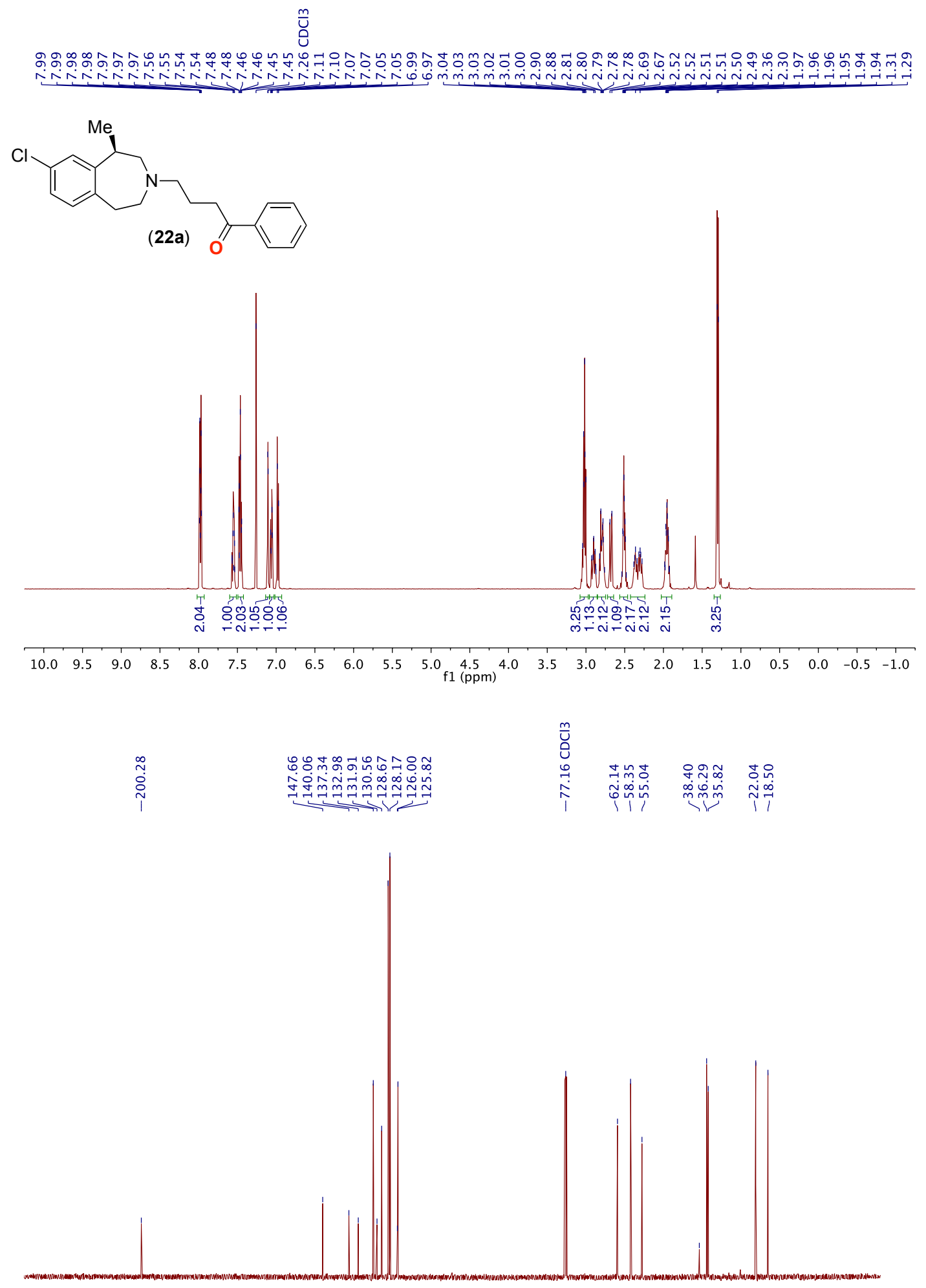

$\begin{array}{llllllllllllllllllllllllllllll}230 & 220 & 210 & 200 & 190 & 180 & 170 & 160 & 150 & 140 & 130 & 120 & 110 & 100 & 90 & 80 & 70 & 60 & 50 & 40 & 30 & 20 & 10 & 0 & -10\end{array}$ 
${ }^{1} \mathrm{H}-\mathrm{NMR}$ and ${ }^{13} \mathrm{C}-\mathrm{NMR}$ Spectra for compound 23a
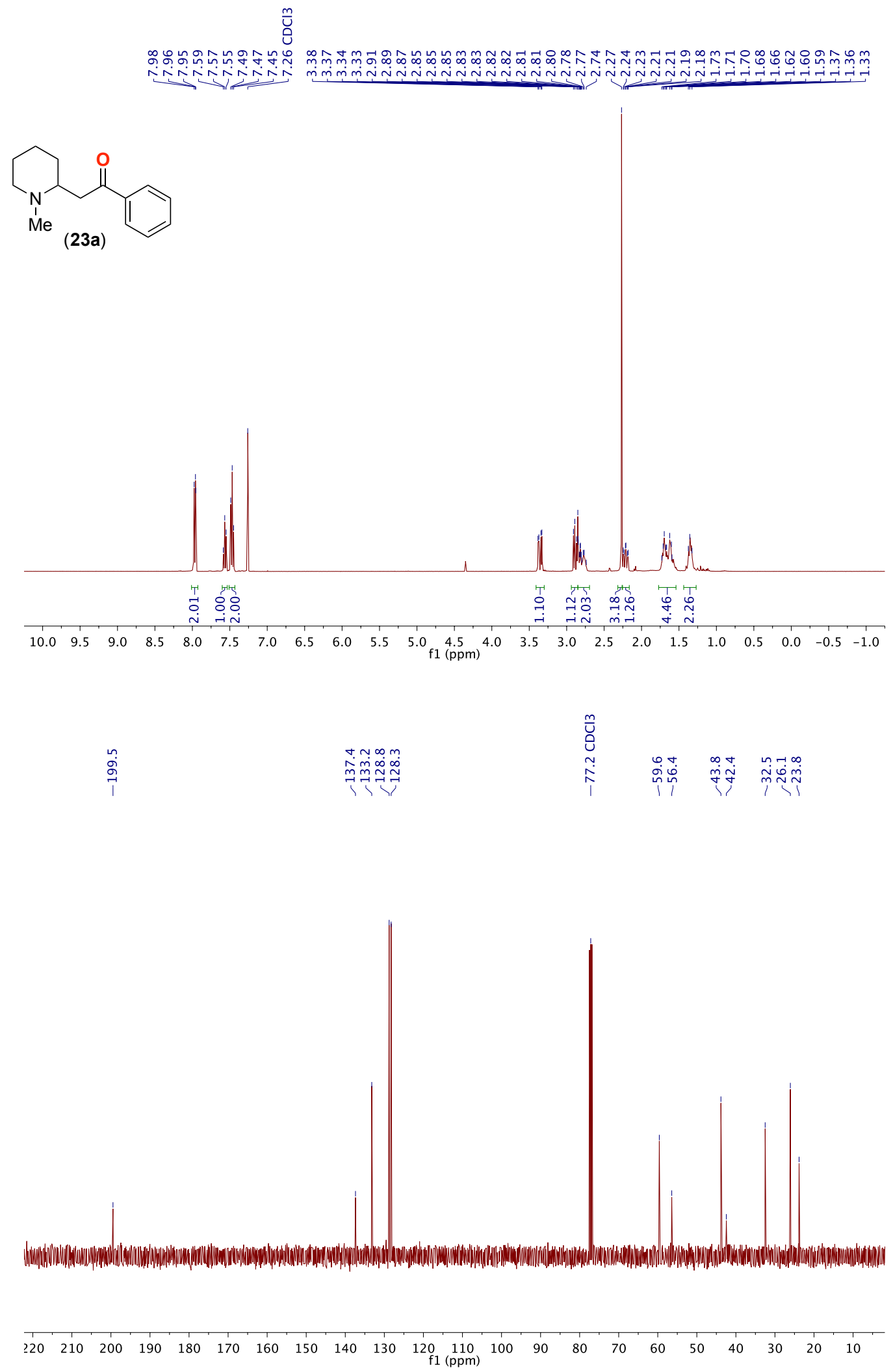
${ }^{1}$ H-NMR, ${ }^{13} \mathrm{C}$-NMR and ${ }^{19}$ F-NMR Spectra for compound 24a
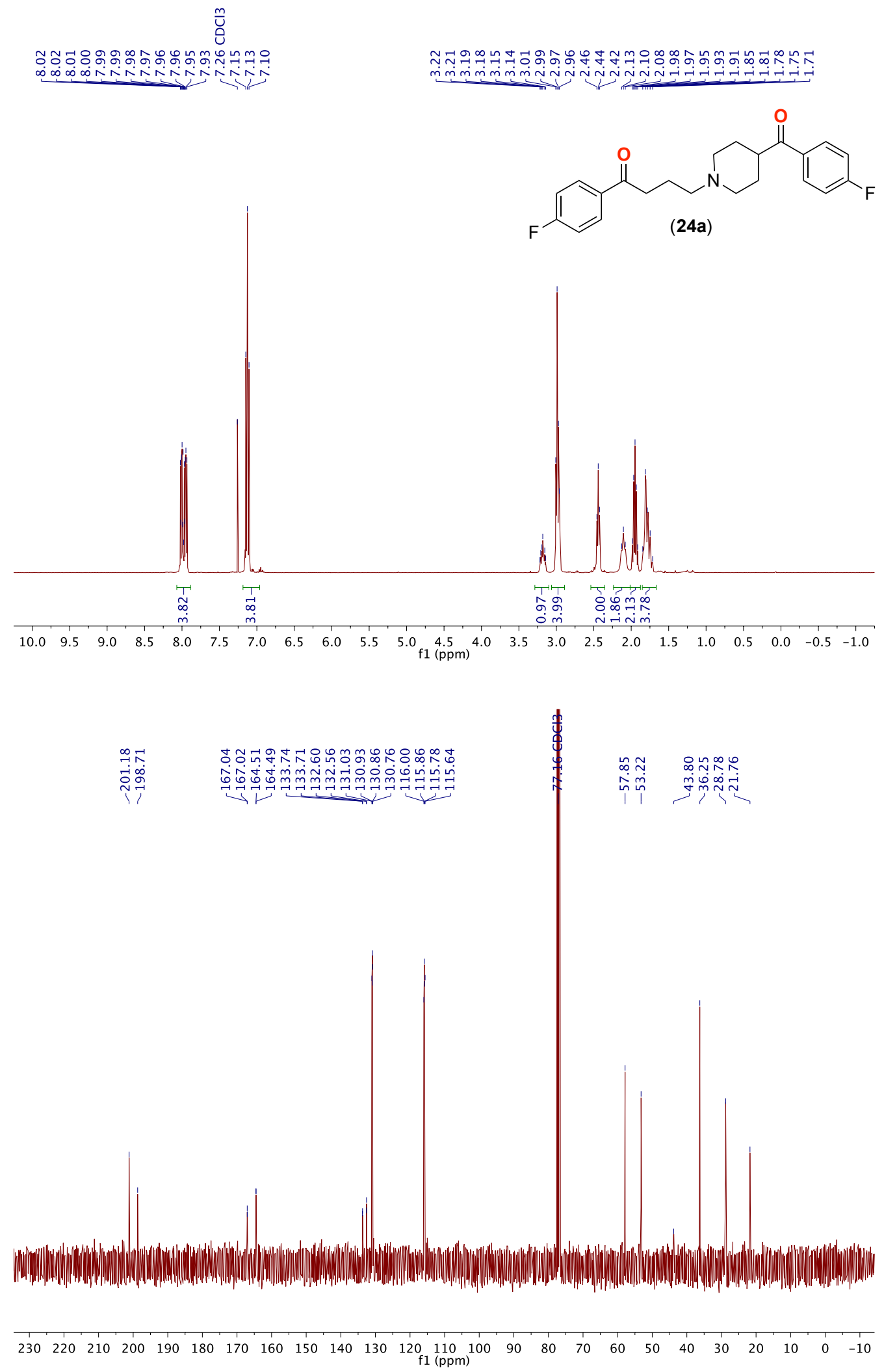


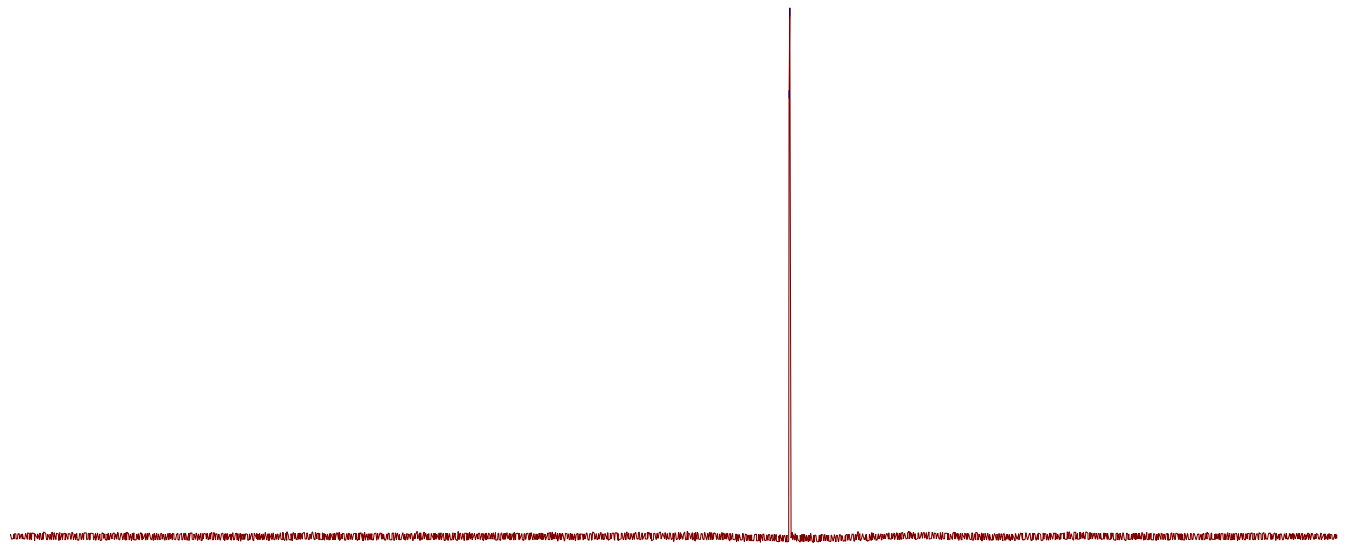

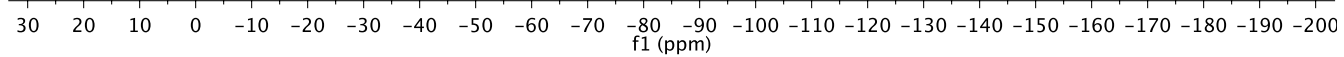


${ }^{1} \mathrm{H}-\mathrm{NMR},{ }^{13} \mathrm{C}-\mathrm{NMR}$ Spectra and ${ }^{19} \mathrm{~F}-\mathrm{NMR}$ for compound 25a
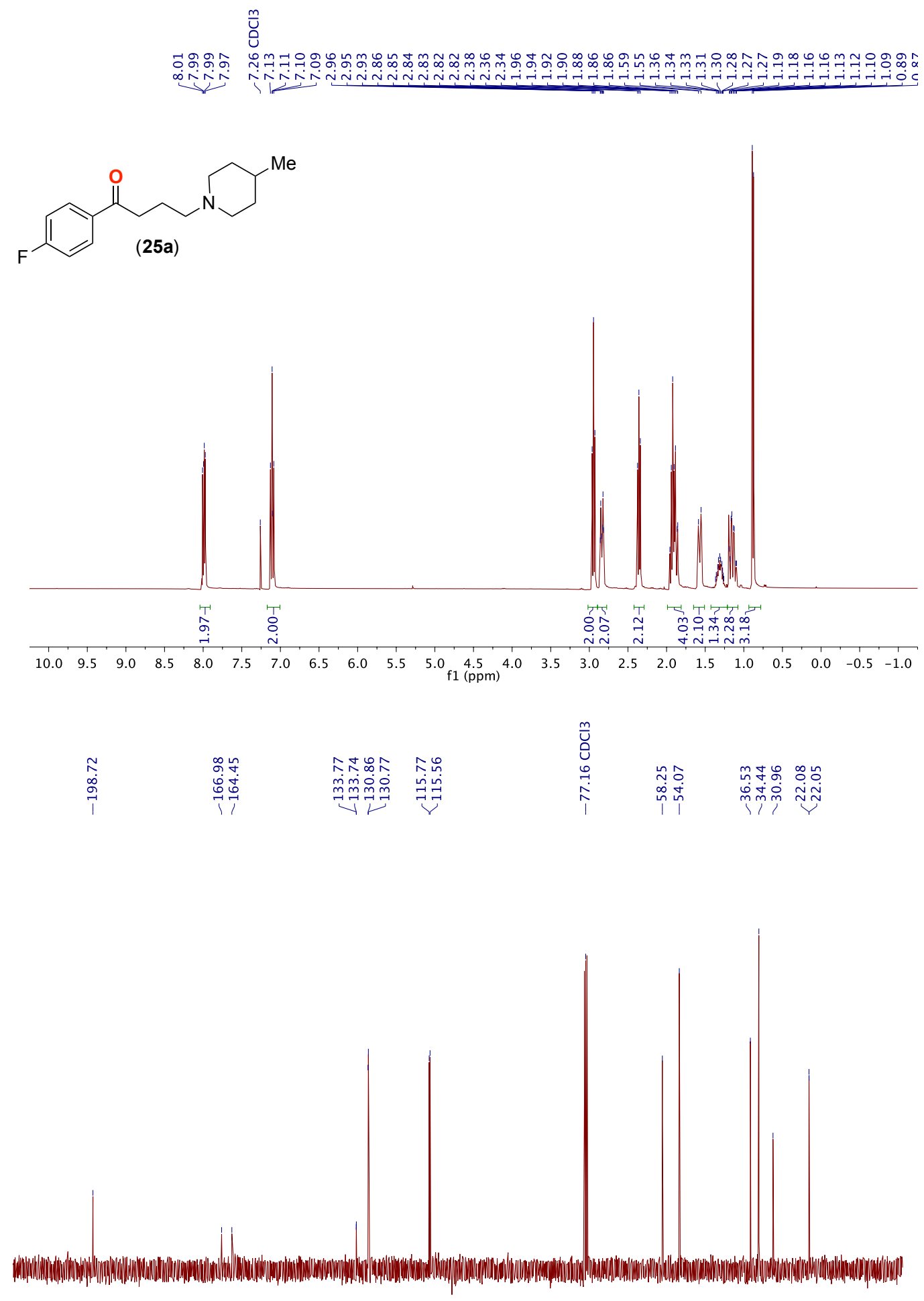

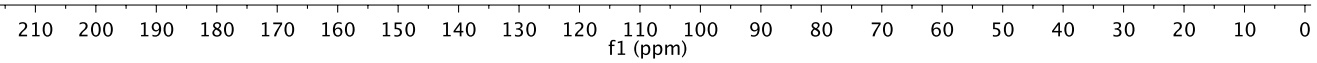




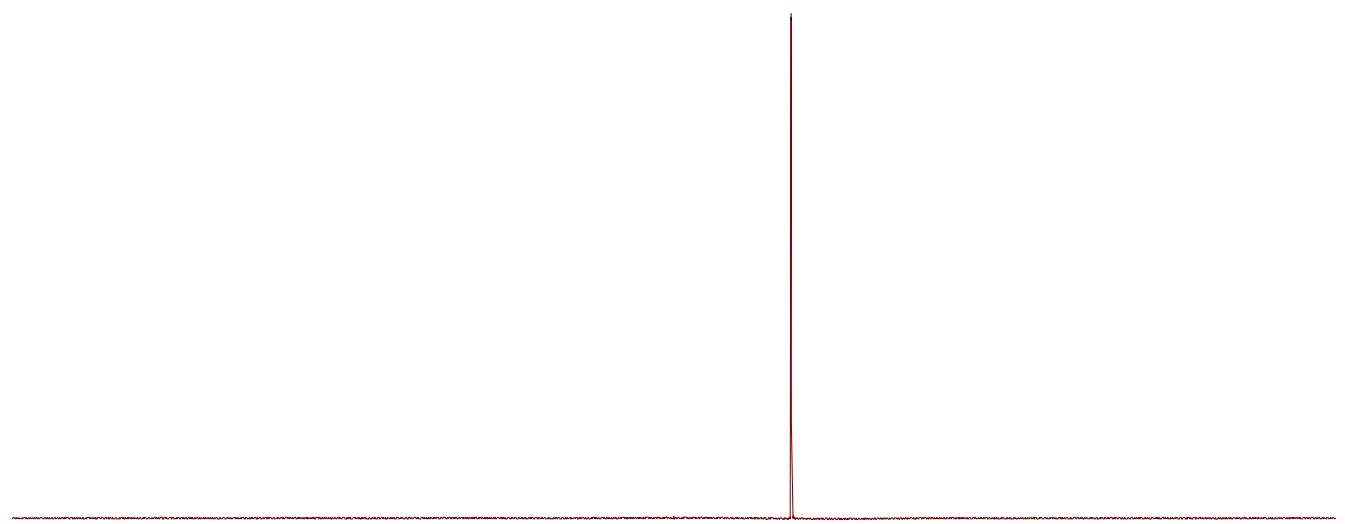

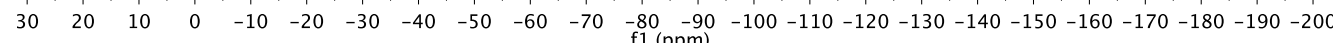


${ }^{1} \mathrm{H}-\mathrm{NMR},{ }^{13} \mathrm{C}-\mathrm{NMR}$ and ${ }^{19} \mathrm{~F}-\mathrm{NMR}$ Spectra for compound 26a
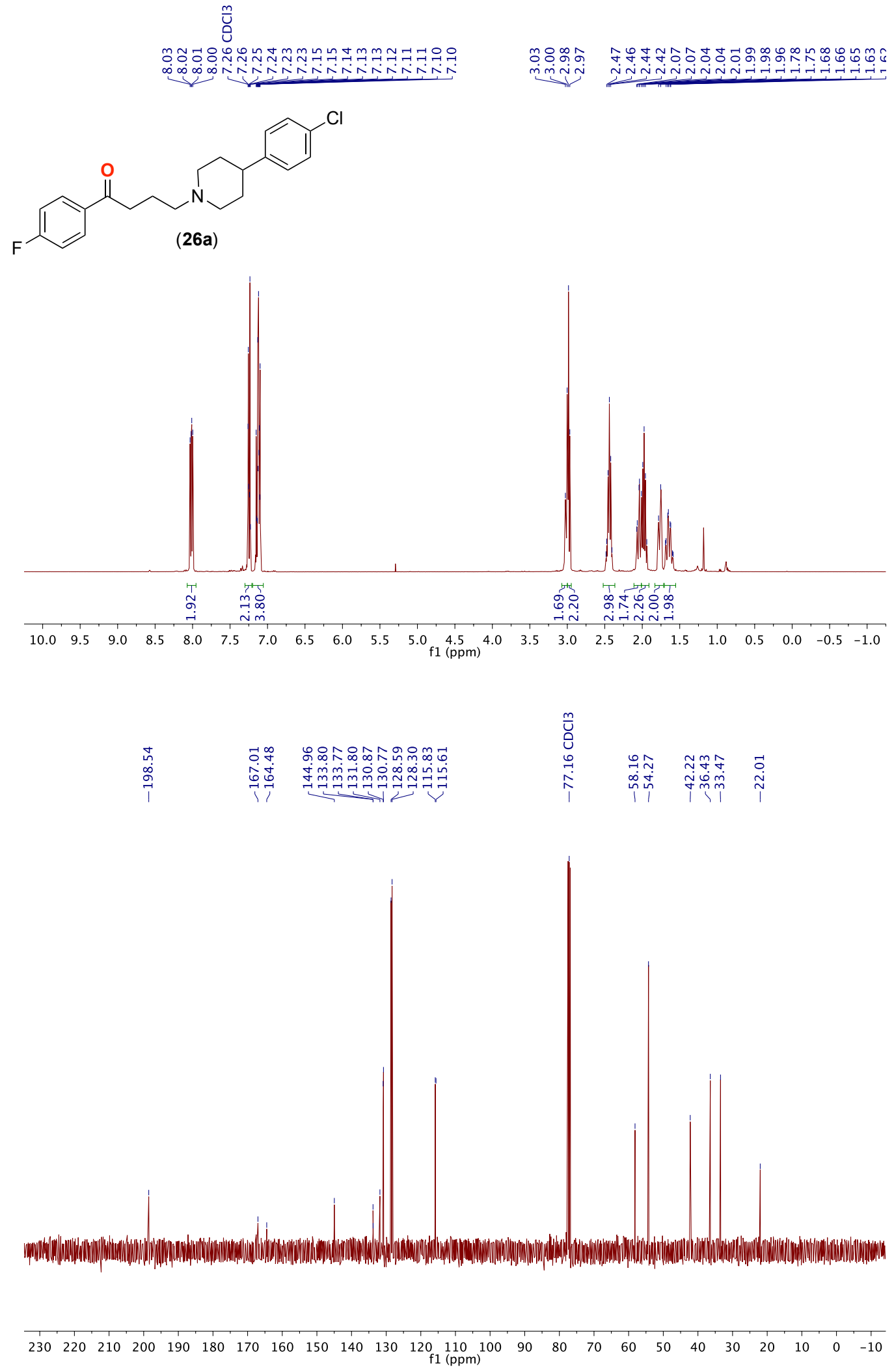


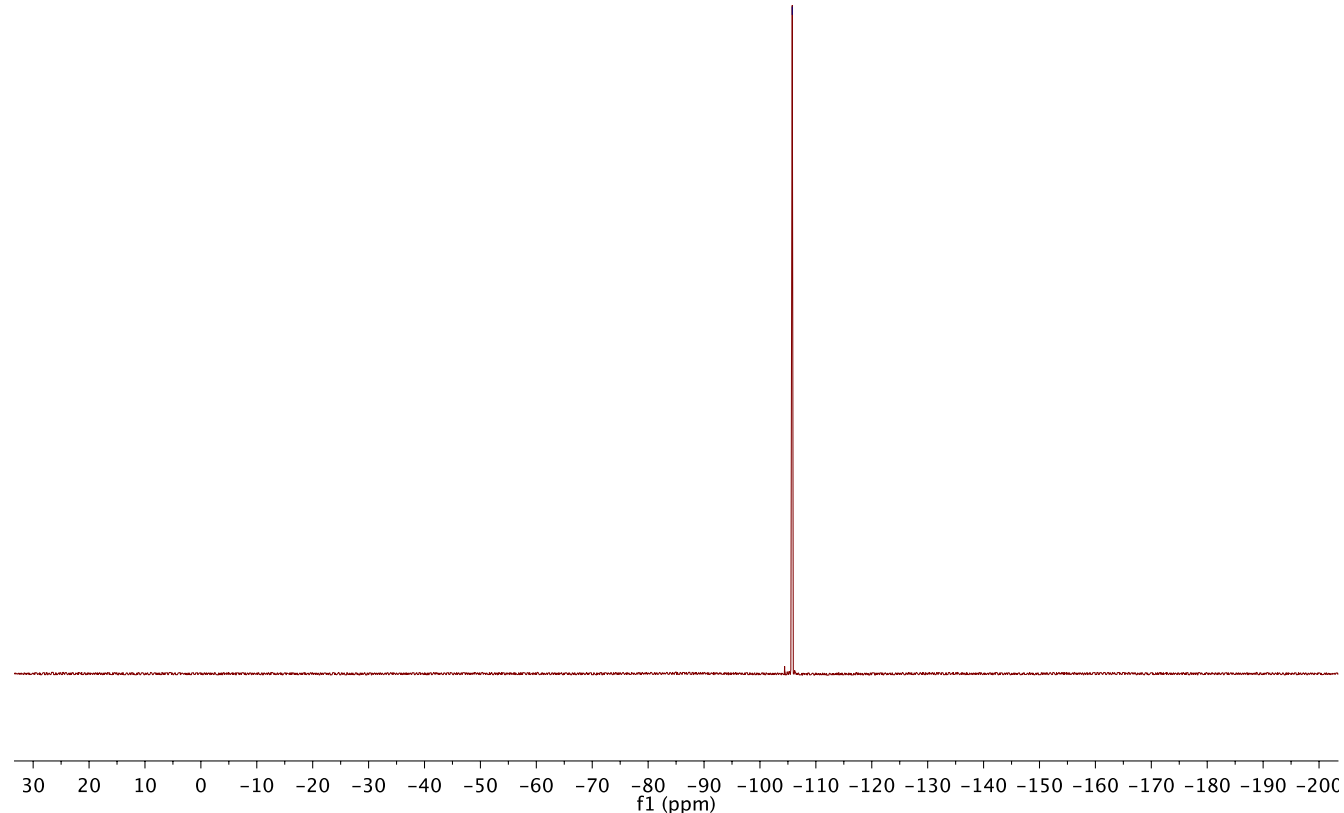


${ }^{1} \mathrm{H}-\mathrm{NMR}$ and ${ }^{13} \mathrm{C}$-NMR Spectra for compound 27a

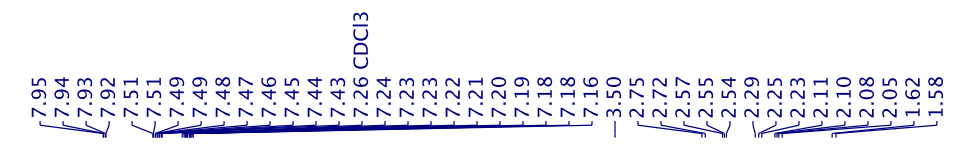

(27a)
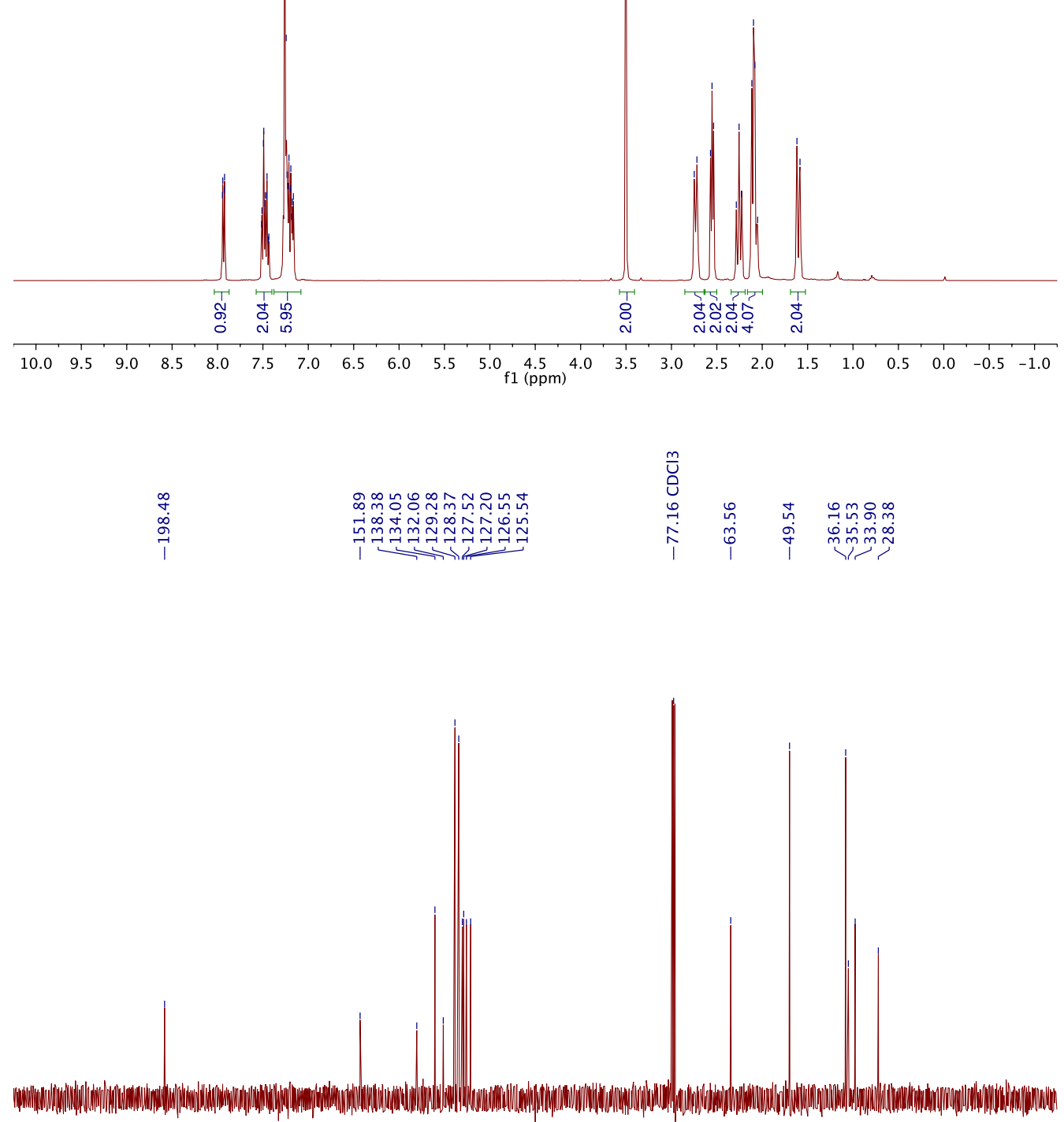

$\begin{array}{lllllllllllllllllllllllllllllllllll}230 & 220 & 210 & 200 & 190 & 180 & 170 & 160 & 150 & 140 & 130 & 120 & 110 & 100 & 90 & 80 & 70 & 60 & 50 & 40 & 30 & 20 & 10 & 0 & -10\end{array}$ 